\title{
Development of Photolenalidomide for Cellular Target Identification
}

\author{
Zhi Lin, ${ }^{1,}{ }^{\dagger}$ Yuka Amako, ${ }^{1,}{ }^{\dagger}$ Farah Kabir, ${ }^{1}$ Hope A. Flaxman, ${ }^{1}$ Bogdan Budnik, ${ }^{2}$ Christina M.
}

Woo ${ }^{1, *}$

${ }^{1}$ Department of Chemistry and Chemical Biology, Harvard University, Cambridge, Massachusetts 02138 United States

${ }^{2}$ Mass Spectrometry and Proteomics Resource (MSPRL), Division of Science, Faculty of Arts and Sciences, Harvard University, Cambridge, Massachusetts 02138 United States

$\dagger$ These authors contributed equally to this work

*Email: cwoo@chemistry.harvard.edu

Index

Additional Experimental Information.

Scheme S1 | Synthetic scheme for photolenalidomide and analogues 1-6, Me-Len and MepLen.

Figure S1 | Levels of selected substrates of the IMiD analogues following treatment in MM.1S cells.

Figure S2 | Antiproliferative effects of lenalidomide and its analogues.

Figure S3 | Immunomodulatory response of interleukin-2 (IL-2) and CD69.

Figure S4 | Binding site mapping of photolenalidomide with the thalidomide-binding domain of CRBN (CULT domain).

Figure S5 | Docked structure of the DDB1/CRBN-Len-GSPT1 complex.

Figure S6 | Photolenalidomide used to label and enrich lenalidomide targets in live cells.

Figure S7 | elF3i forms a complex with lenalidomide and CRBN without enhanced ubiquitylation or degradation.

Figure S8 | Uncropped immunoblots related to Figure 1c.

Figure S9 | Uncropped immunoblots related to Figure $\mathbf{3 a}$ and Figure $\mathbf{3 d}$.

Figure S10 | Uncropped immunoblots related to Figure 4b, Figure 4c and Figure 4e.

Figure S11 | Uncropped immunoblots related to Figure $\mathbf{4 f}$ and Figure $\mathbf{4 g}$.

Figure S12 | Uncropped immunoblots related to Figure 5a, Figure 5c and Figure 5d.

Table S1 | Complete list of pLen-modified peptide spectral matches identified by LC-MS/MS.

Table S2 | Unique pLen-modified peptide assignments identified by LC-MS/MS.

Table S3 | Complete list of analogue 1-modified peptide spectral matches identified by LCMS/ MS.

Table S4 | Unique analogue 1-modified peptide assignments identified by LC-MS/MS.

Table S5 | Complete protein list identified by LC-MS/MS in Figure 3c.

Table S6 | Complete protein list identified by LC-MS/MS in Figure 4a.

Table S7 | Complete protein list identified by LC-MS/MS in Figure 4d. 


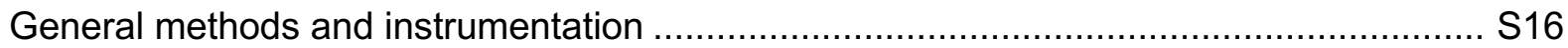

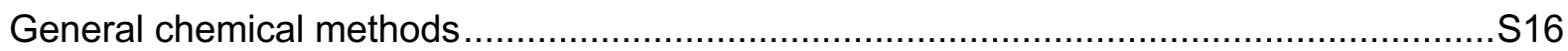

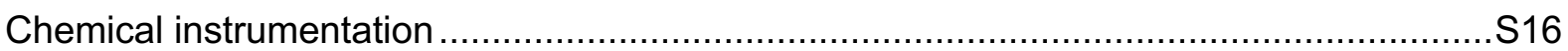

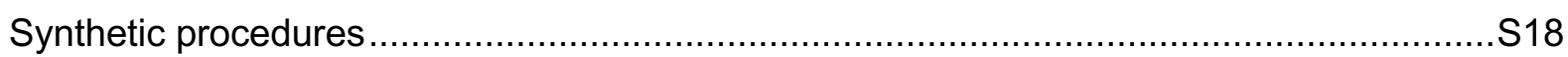

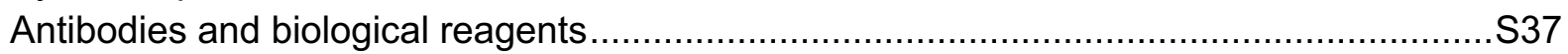

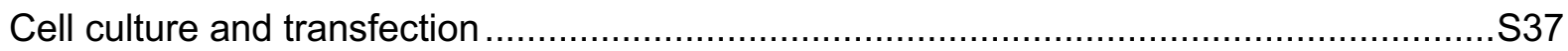

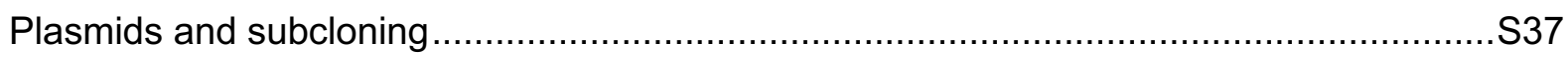

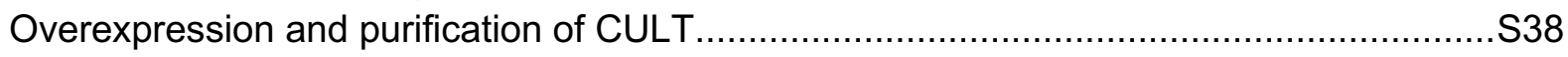

Structural proteomics mass spectrometry sample preparation ......................................... 39

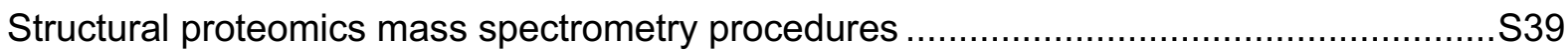

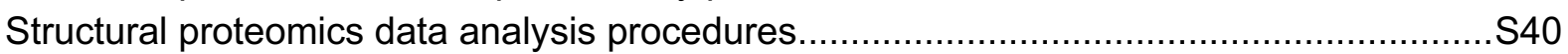

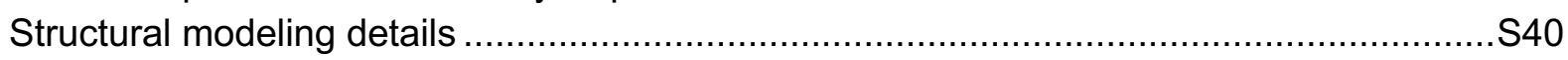

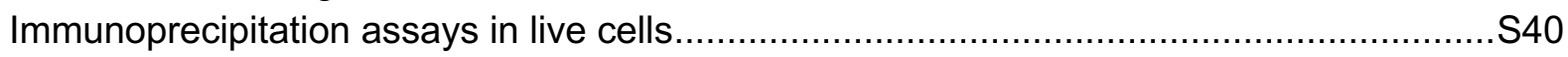

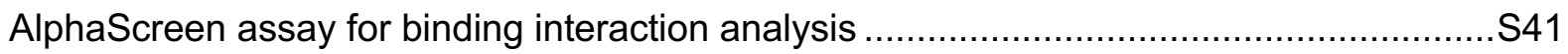

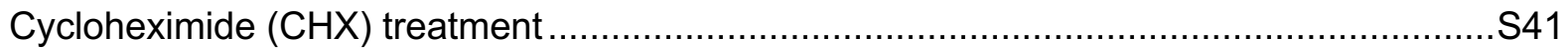

Photoaffinity labeling experiment and in-gel fluorescent imaging....................................... 44

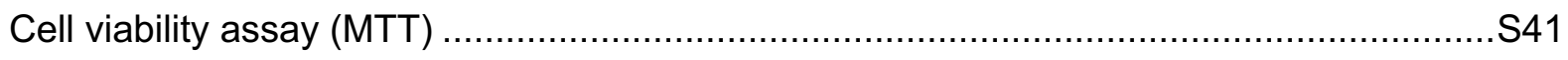

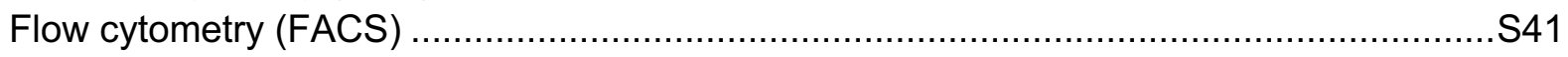

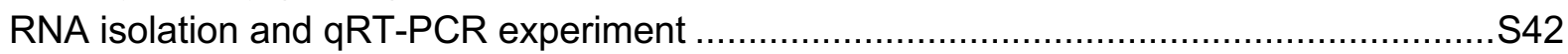

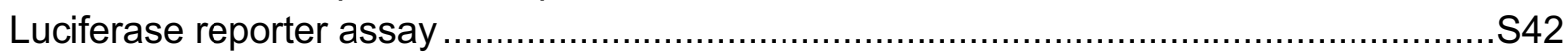

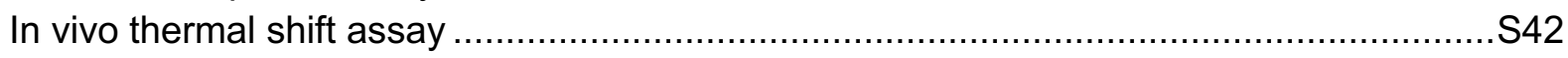

Photoaffinity labeling and enrichment of lenalidomide targets in living cells and on-bead

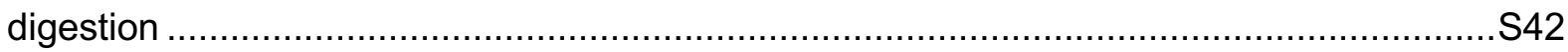

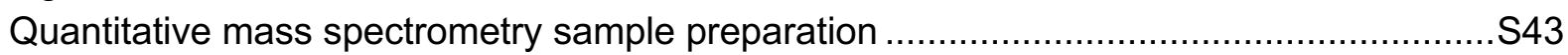

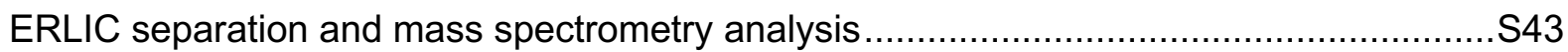

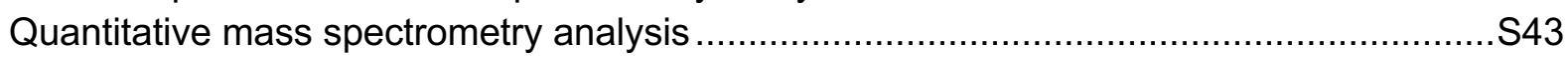

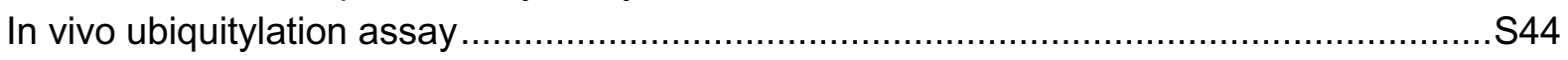

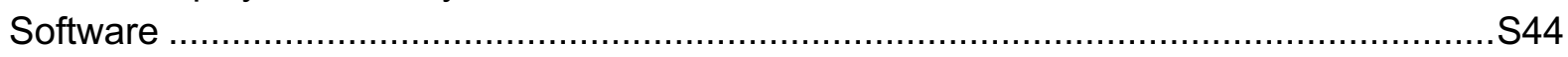

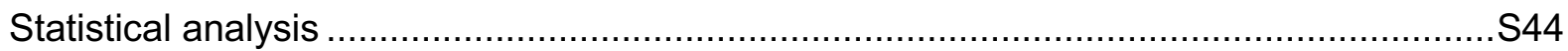

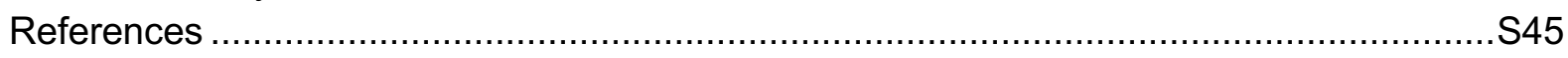

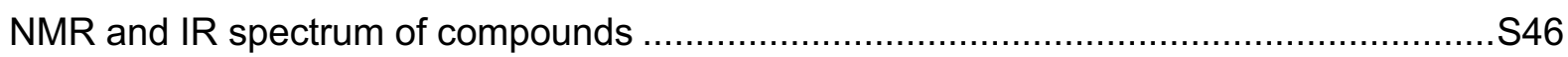


SUPPORTING INFORMATION FIGURES

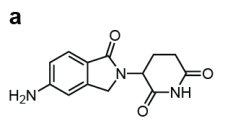

s1

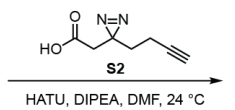

$20 \%$

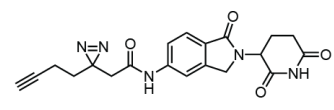

photolenalidomide

b

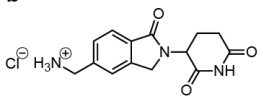

s3
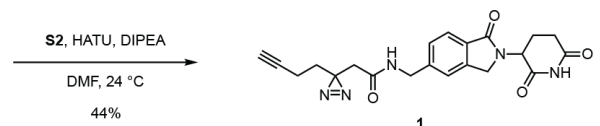

c

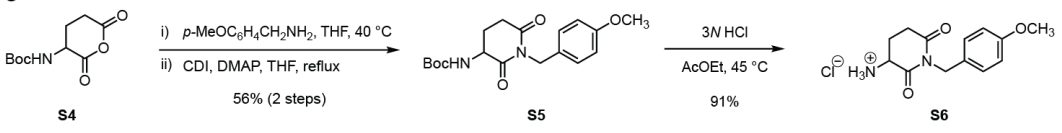

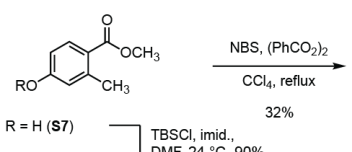

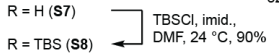

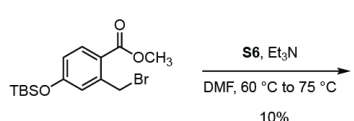

s9

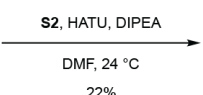

$22 \%$

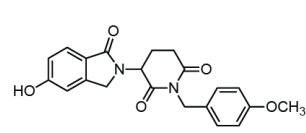

$\mathbf{s} 10$

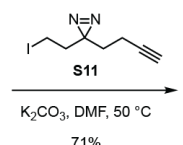

$71 \%$
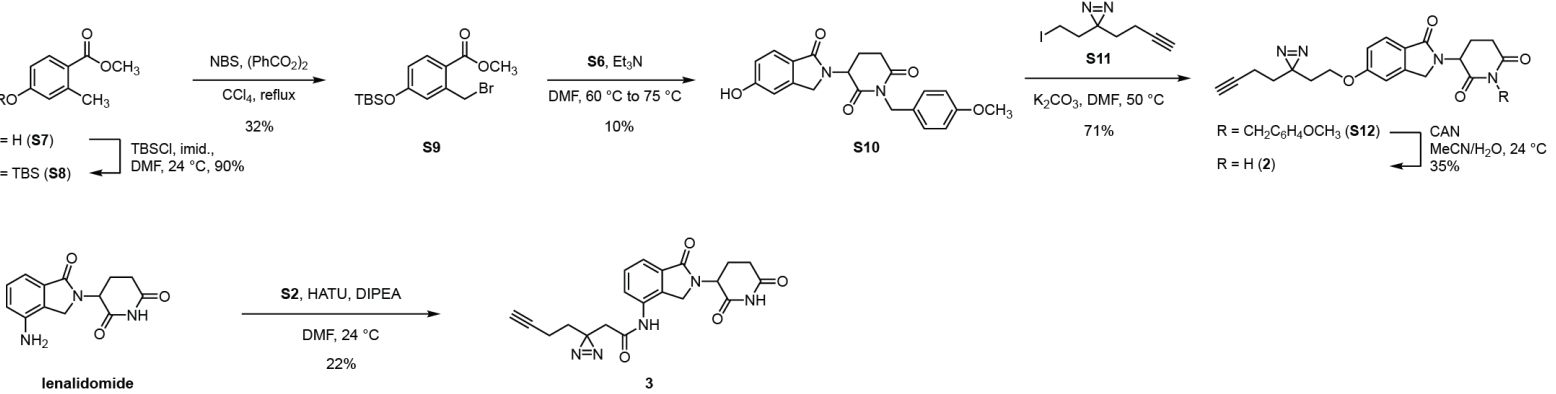

e

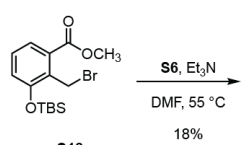

S13

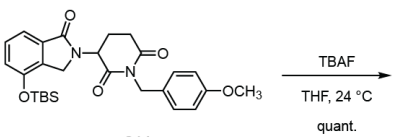

$\$ 14$

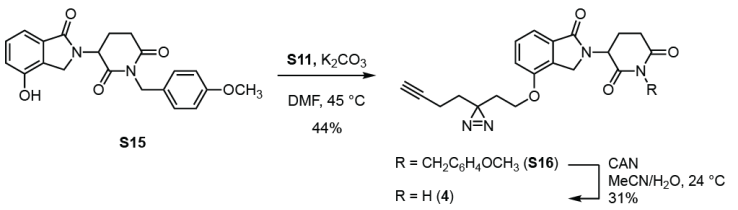

f
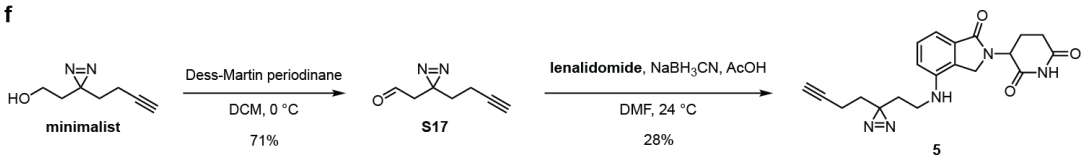

g

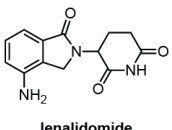

lenalidomide

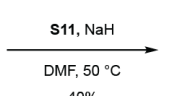

$40 \%$

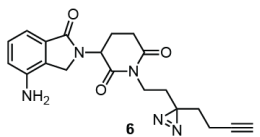

h

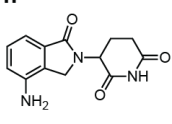

lenalidomide

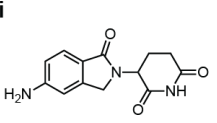

s1

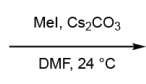

quant.

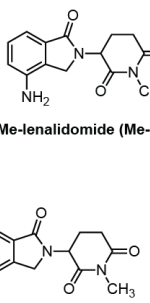

S18

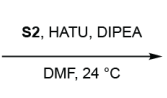

$11 \%$

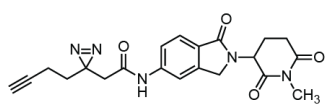

Me-photolenalidomide (Me-pLen)

Scheme S1 | Synthetic scheme for photolenalidomide, analogues 1-6, Me-Len and MepLen. Synthesis of (a) photolenalidomide, (b) analogue 1, (c) analogue 2, (d) analogue 3, (e) analogue 4, (f) analogue 5, (g) analogue 6, (h) Me-lenalidomide and (i) Mephotolenalidomide.

S3 
a

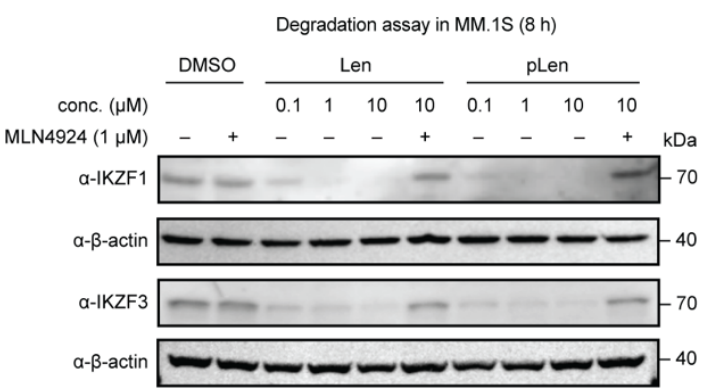

b

Degradation assay in MM.1S ( $8 \mathrm{~h}$ )

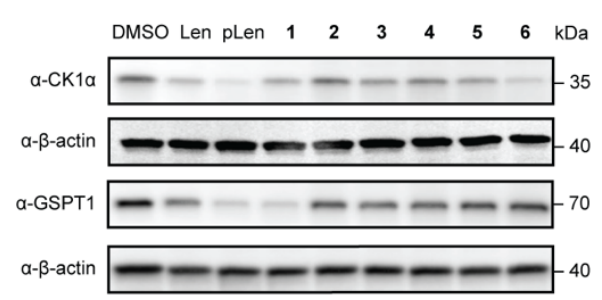

C

Thermal shift assay in MM.1S

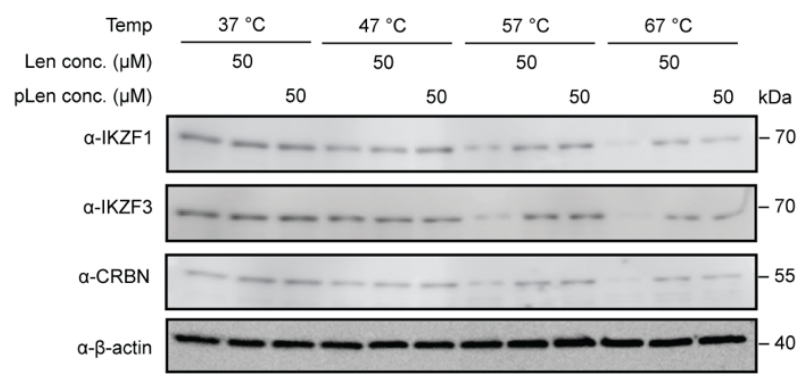

Figure S1 | Levels of selected substrates of the IMiD analogues following treatment in MM.1S cells. (a) Western blot of IKZF1 and IKZF3 after treatment of MM.1S cells with the indicated concentration of Len or pLen for $8 \mathrm{~h}$ with or without $1 \mu \mathrm{M} \mathrm{MLN4924.} \mathrm{(b)} \mathrm{Western} \mathrm{blot} \mathrm{of} \mathrm{CK1} \alpha$ and GSPT1 after treatment of MM.1S cells with $10 \mu \mathrm{M}$ Len, pLen or analogues 1-6 for $8 \mathrm{~h}$. Results are representative of three biologically independent experiments. (c) Thermal shift assay for IKZF1, IKZF3 and CRBN in MM1.S cells. The cells were incubated with or without Len or pLen for $1 \mathrm{~h}$ and then incubated at the indicated temperature for $5 \mathrm{~min}$ followed by cell lysis. Cell lysates were cleared by centrifugation before immunoblotting. Results are representative of two biologically independent experiments. 
a

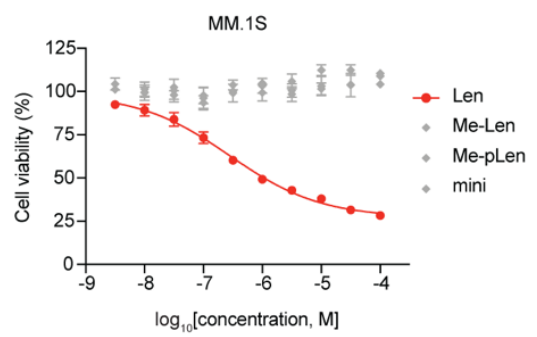

b

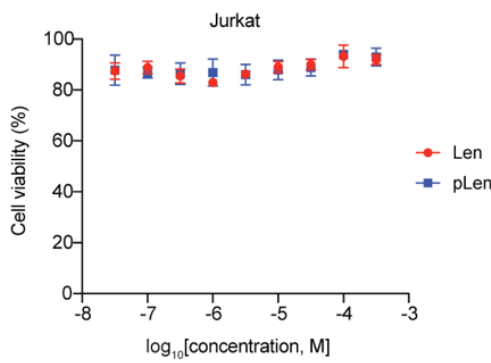

RAW264.7

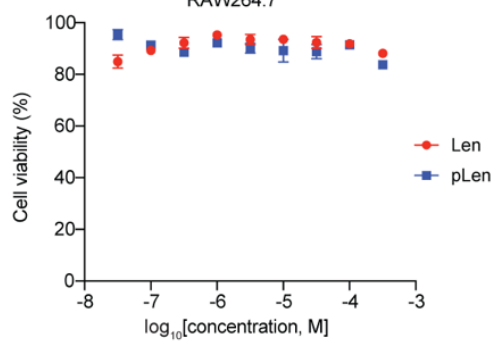

U937
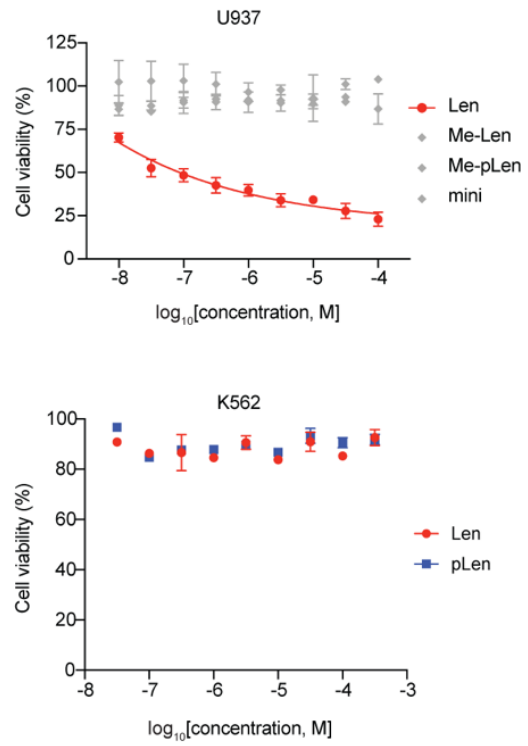

MOLM13

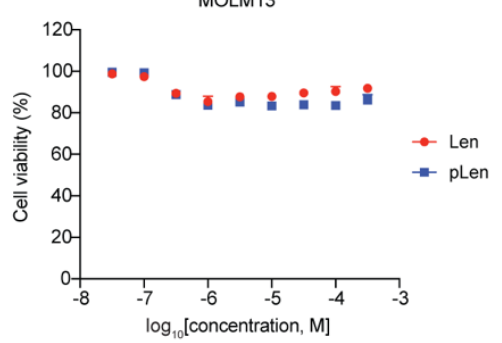

Figure S2 | Antiproliferative effects of lenalidomide and its analogues. (a) Cell viability (MTT) assay of MM.1S or U937 cells after treatment with Len, the analogues Me-Len, Me-pLen, or the minimalist tag (mini) for 5 days. (b) Cell viability assay of Jurkat, K562, RAW264.7 and MOLM13 cells after treatment with Len or pLen for 5 days. Results are representative of two biologically independent experiments. Data were normalized to the untreated samples. All graphs are shown as mean \pm s.d., $n=4$. 
a

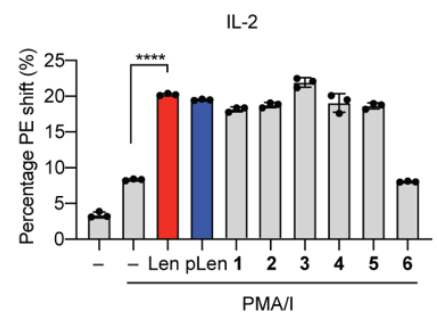

b

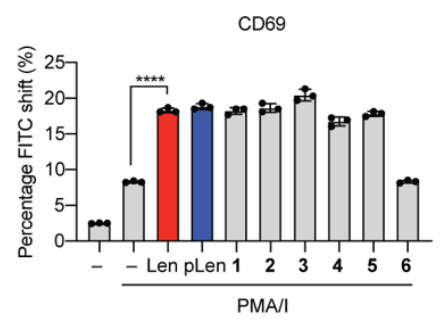

C

Degradation assay in stimulated Jurkat cells

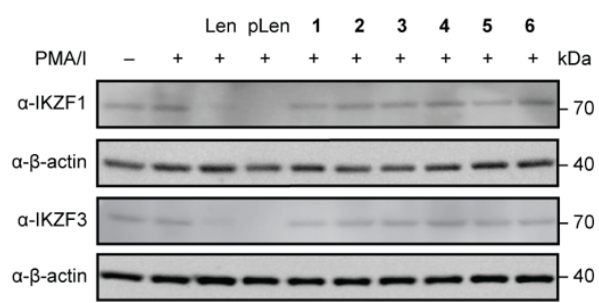

d

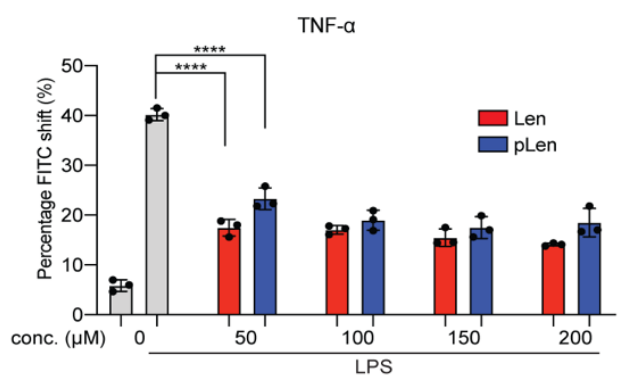

Figure S3 | Immunomodulatory response of interleukin-2 (IL-2) (a) and CD69 (b) in Jurkat cells treated with $1 \mu \mathrm{M}$ Len, pLen, or analogues 1-6. Jurkat cells were treated with the indicated compound $(1 \mu \mathrm{M})$ for $1 \mathrm{~h}$ before stimulation with $1 \mathrm{ng} / \mathrm{mL}$ phorbol 12 -myristate 13 acetate and $1 \mathrm{mg} / \mathrm{mL}$ ionomycin (PMA/I) for $48 \mathrm{~h}$. Data represent the \% IL-2- or CD69-positive cell numbers among total cell counts of 100,000 . Results are representative of four biologically independent experiments. (c) Western blot of IKZF1 and IKZF3 after treatment of Jurkat cells with $10 \mu \mathrm{M}$ Len, pLen, analogues $\mathbf{1 - 6}$ for $1 \mathrm{~h}$ before stimulation with $1 \mathrm{ng} / \mathrm{mL}$ phorbol 12 myristate 13-acetate and $1 \mathrm{mg} / \mathrm{mL}$ ionomycin (PMA/l) for $48 \mathrm{~h}$. Results are representative of two biologically independent experiments. (d) Immunomodulatory response of TNF- $\alpha$ in RAW264.7 cells upon treatment. Levels of TNF- $\alpha$ in RAW264.7 cells were measured by flow cytometry. The cells were treated with Len or pLen at the indicated concentration for $1 \mathrm{~h}$ and stimulated with $1 \mathrm{ng} / \mathrm{mL}$ lipopolysaccharide (LPS) for $3 \mathrm{~h}$ prior to flow cytometry analysis. Results are representative of four biologically independent experiments. Statistical significance was analyzed using a 2-way Student's $t$-test. ${ }^{* * *} \mathrm{P}<0.0001$. 
a

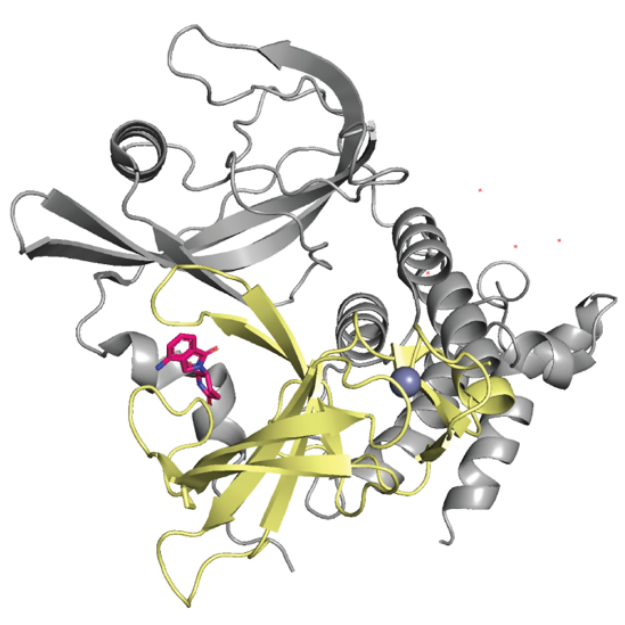

b

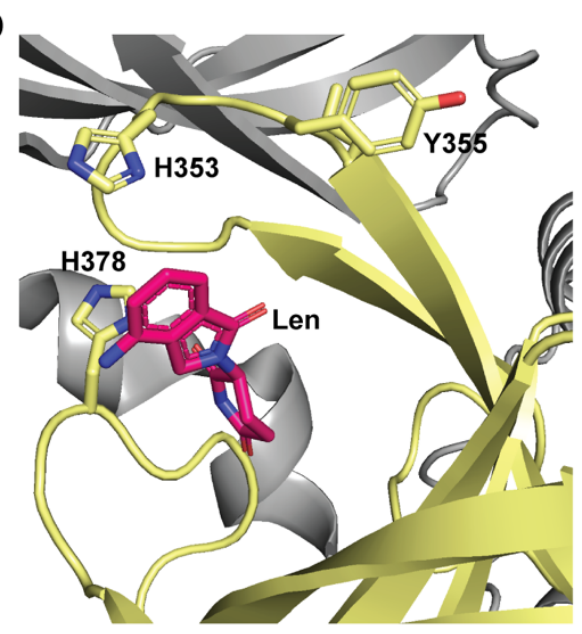

C

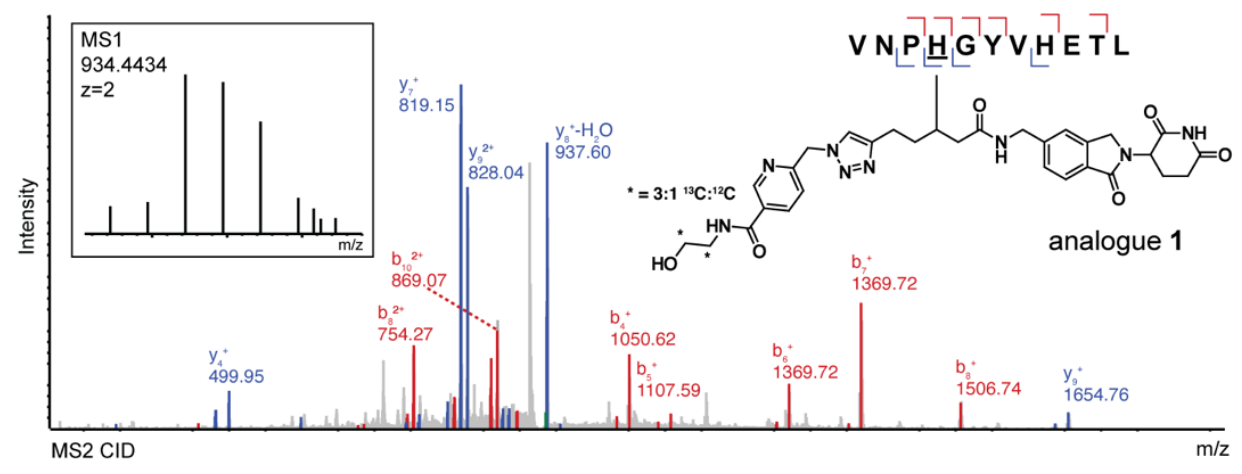

Figure S4 | Binding site mapping of photolenalidomide with the thalidomide-binding domain of CRBN (CULT domain). (a) Structure of the DDB1-CRBN-lenalidomide complex (PDB 4CI2, DDB1 is not shown) with the CULT domain highlighted in yellow and the rest of CRBN in grey. (b) Enlarged view of the tri-tryptophan binding pocket in the thalidomide-binding domain of CRBN. Side chains of amino acids modified by photolenalidomide (His353 and Tyr355) or analogue 1 (His353 and His378) are shown in stick form. (c) Representative assignment of a peptide in CULT conjugated to analogue 1. 
a

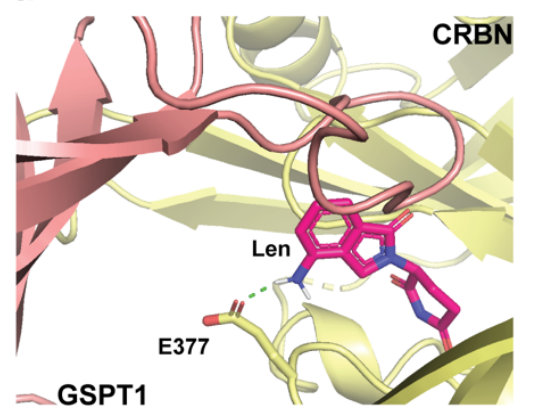

b

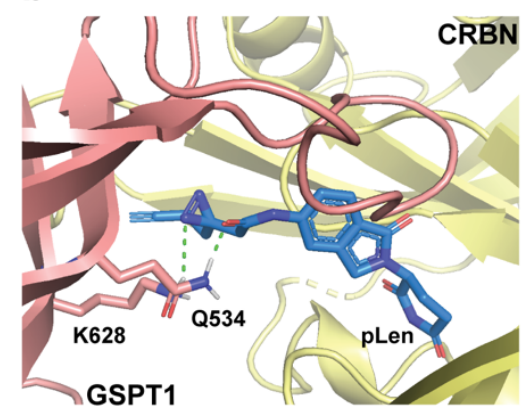

C

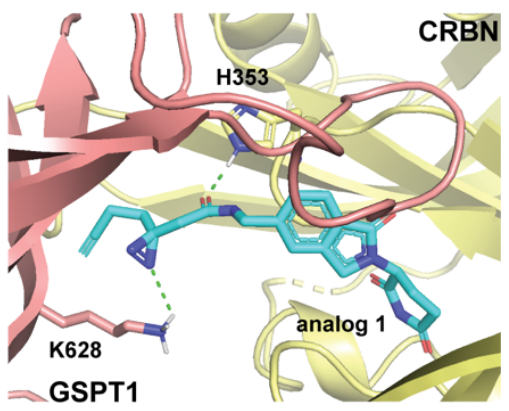

Figure S5 | Docked structures of the (a) DDB1/CRBN-Len-GSPT1 complex, (b) DDB1/CRBN-pLen-GSPT1 complex and (c) DDB1/CRBN-analogue 1-GSPT1 complex adapted from PDB 5HXB. DDB1 is not shown. CRBN is colored yellow and GSPT1 is colored pale pink. 
a CRBN co-IP in HEK293T-CRBN cells

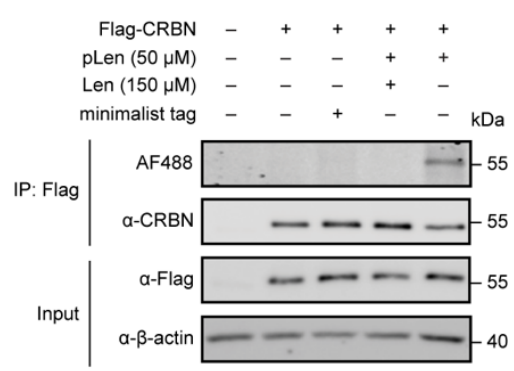

b

PAL enrichment in MM.1S cells

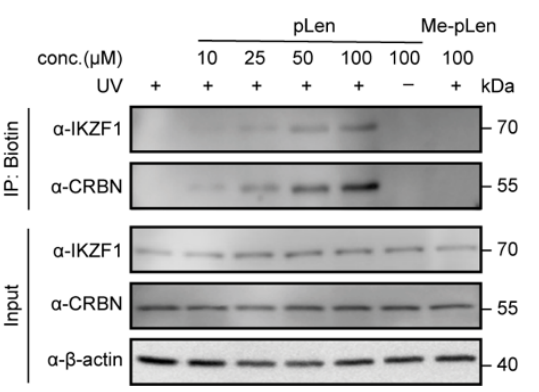

Figure S6 | Photolenalidomide used to label and enrich lenalidomide targets in live cells. (a) In-gel fluorescence imaging of protein targets conjugated with photolenalidomide in HEK293TCRBN cells. HEK293T-CRBN cells were treated with the indicated compound(s) for $1 \mathrm{~h}$ prior to photoirradiation and lysis. The target proteins were immunoprecipitated, subjected to click chemistry with AF488-azide, and visualized by in-gel fluorescence imaging or Western blot analysis. (b) Western blot of IKZF1 and CRBN following treatment of MM.1S cells with the indicated compound pLen or Me-pLen at the indicated concentration, photoirradiation, and enrichment. Results are representative of two biologically independent experiments. 


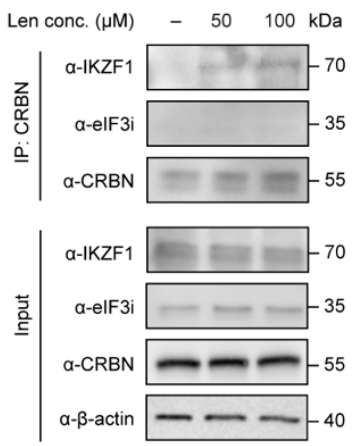

b

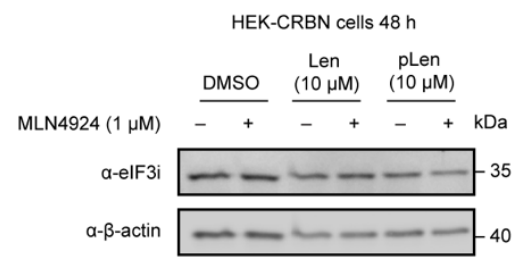

d

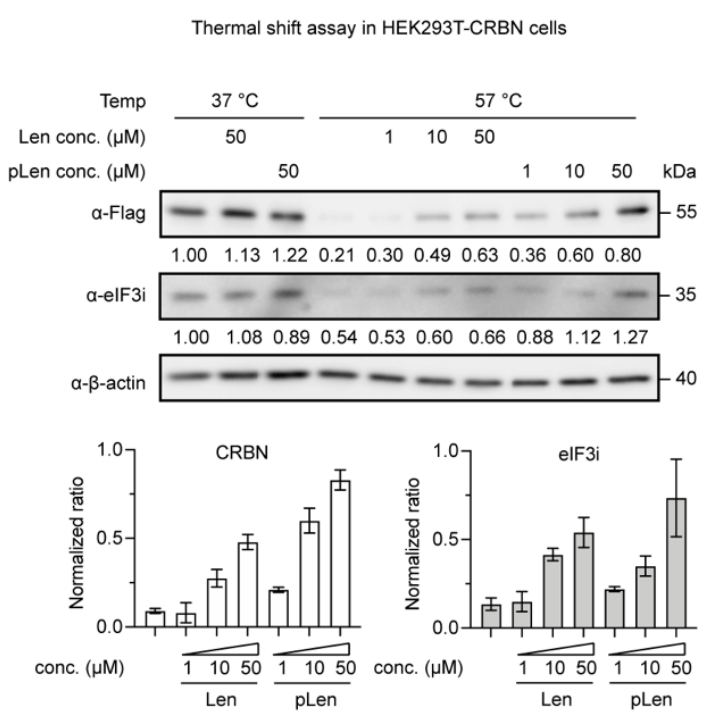

C
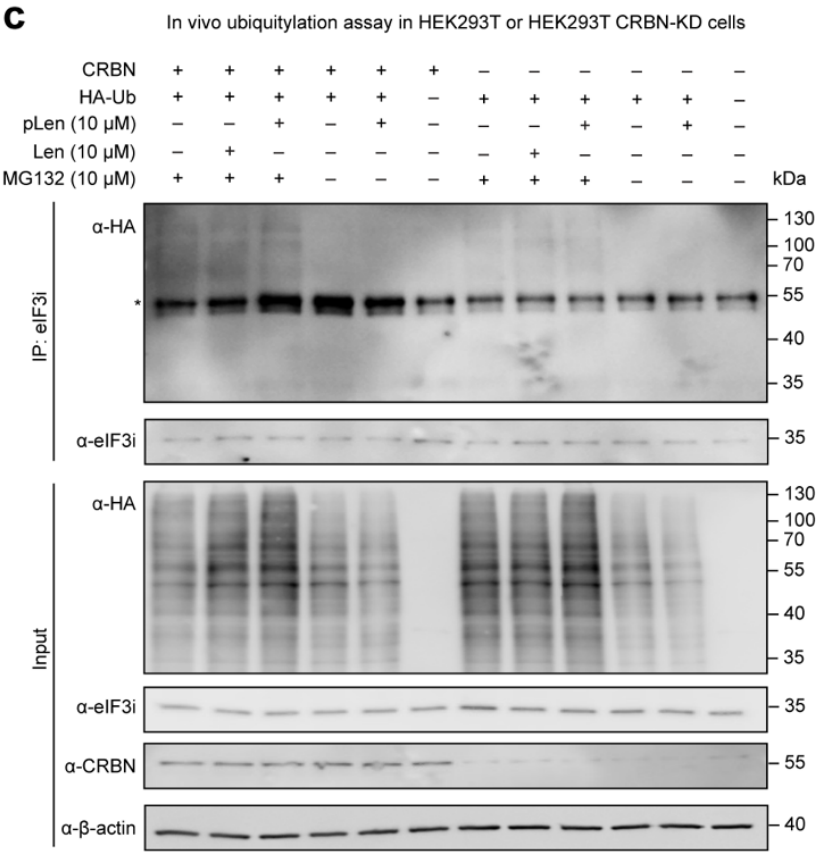

e
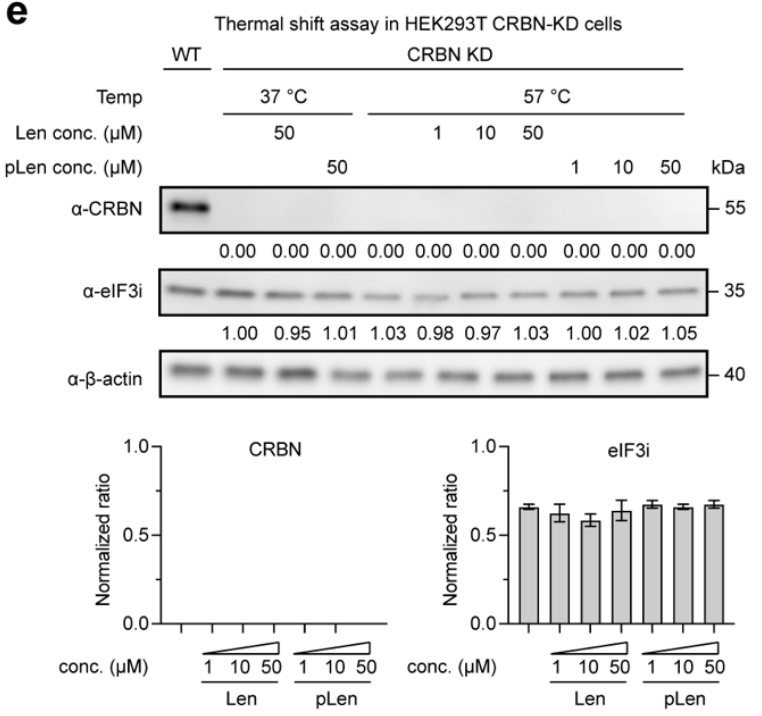

Figure S7 | elF3i forms a complex with lenalidomide and CRBN without enhanced ubiquitylation or degradation. (a) Coimmunoprecipitation of elF3i in MM.1S cells by endogenous CRBN with or without lenalidomide. MM.1S cells were treated with lenalidomide at the indicated concentration for $1 \mathrm{~h}$ prior to lysis. The target proteins were immunoprecipitated and visualized by immunoblotting. Results are representative of three biologically independent experiments. (b) Western blot of elF3i after treatment with Len or pLen in HEK293T-CRBN cells for $48 \mathrm{~h}$ with and without $1 \mu \mathrm{M}$ MLN4924. (c) In vivo ubiquitylation assay in HEK293T or HEK293T CRBN-KD cells. Cells transfected with HA-Ub were treated with $10 \mu \mathrm{M}$ Len or pLen for $1 \mathrm{~h}$ before $10 \mu \mathrm{M}$ MG132 was added. After $6 \mathrm{~h}$ incubation, cells were collected, immunoprecipitated with elF3i antibody, and analyzed by immunoblotting. Input represents the whole cell lysate. The asterisk $\left(^{*}\right)$ denotes a nonspecific band of IgG HC of elF3i antibody. (d, e) Thermal shift assay for Flag-CRBN and elF3i in (d) HEK293T-CRBN cells and (e) HEK293T CRBN-KD cells. The cells were incubated with or without Len or pLen for $1 \mathrm{~h}$ and then incubated at the indicated temperature for 5 min followed by cell lysis. Cell lysates were cleared by centrifugation before immunoblotting. Results are representative of two biologically independent experiments. 
Figure 1c
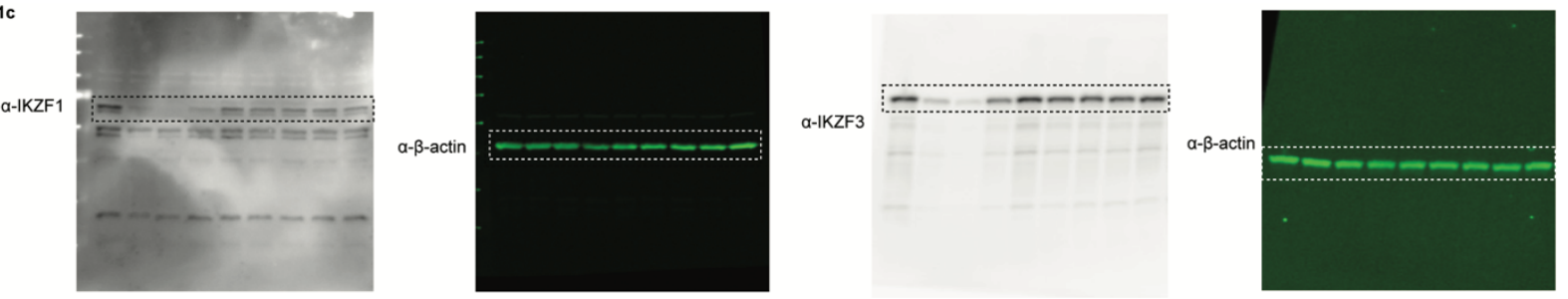

Figure S8 | Uncropped immunoblots related to Figure 1c. 


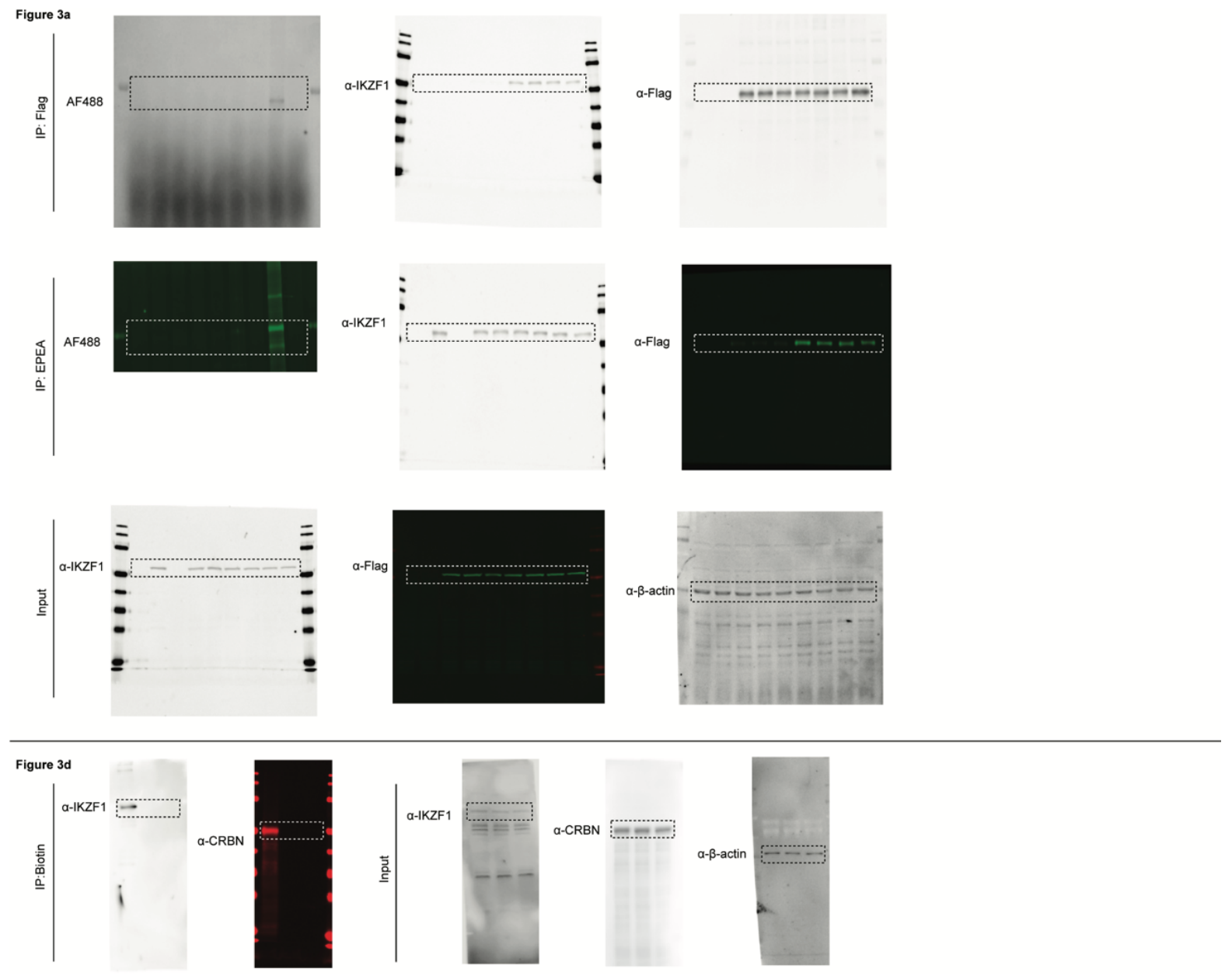

Figure S9 | Uncropped immunoblots related to Figure 3a and Figure 3d. 

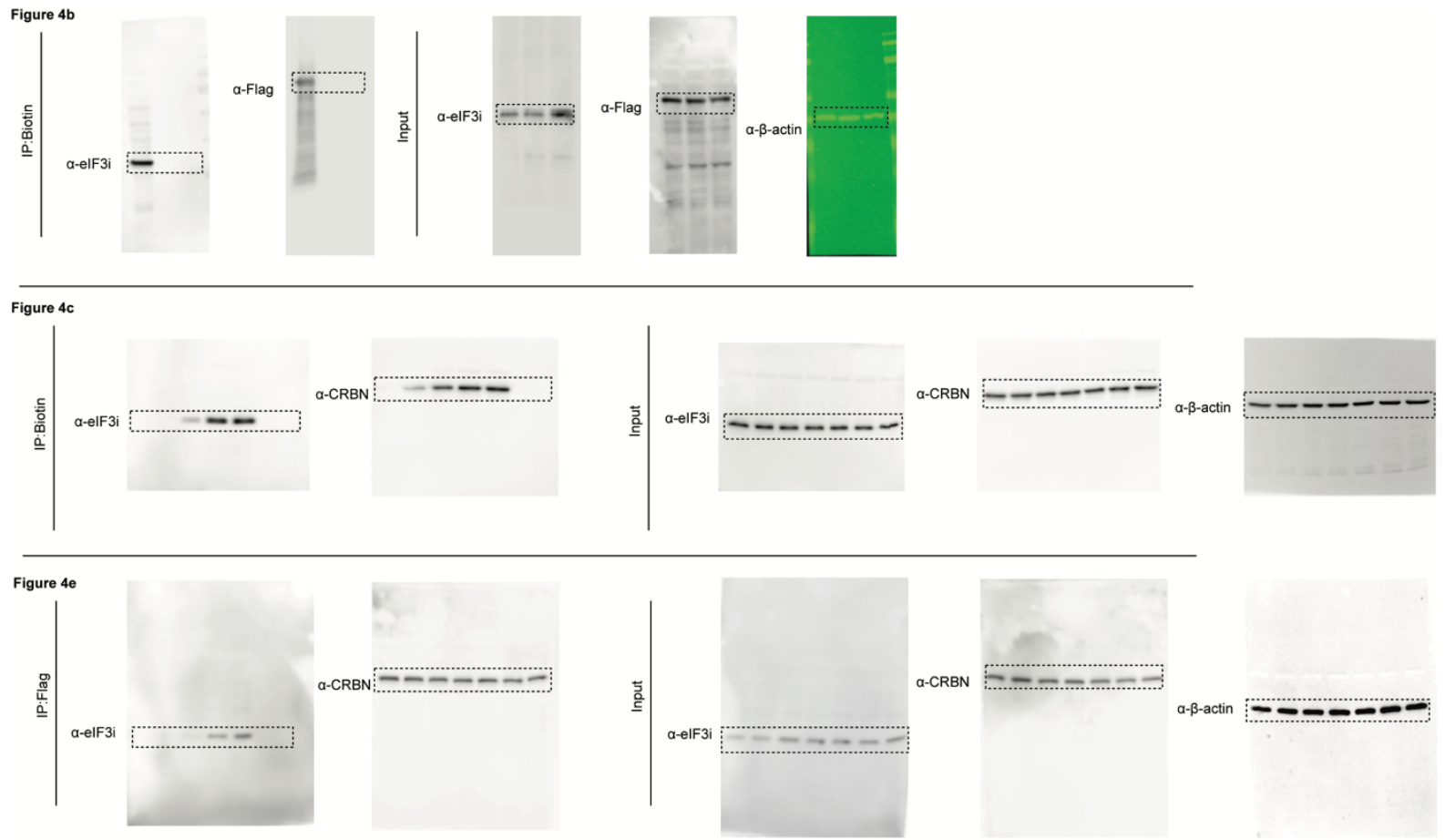

Figure S10 | Uncropped immunoblots related to Figure 4b, Figure 4c and Figure 4e. 

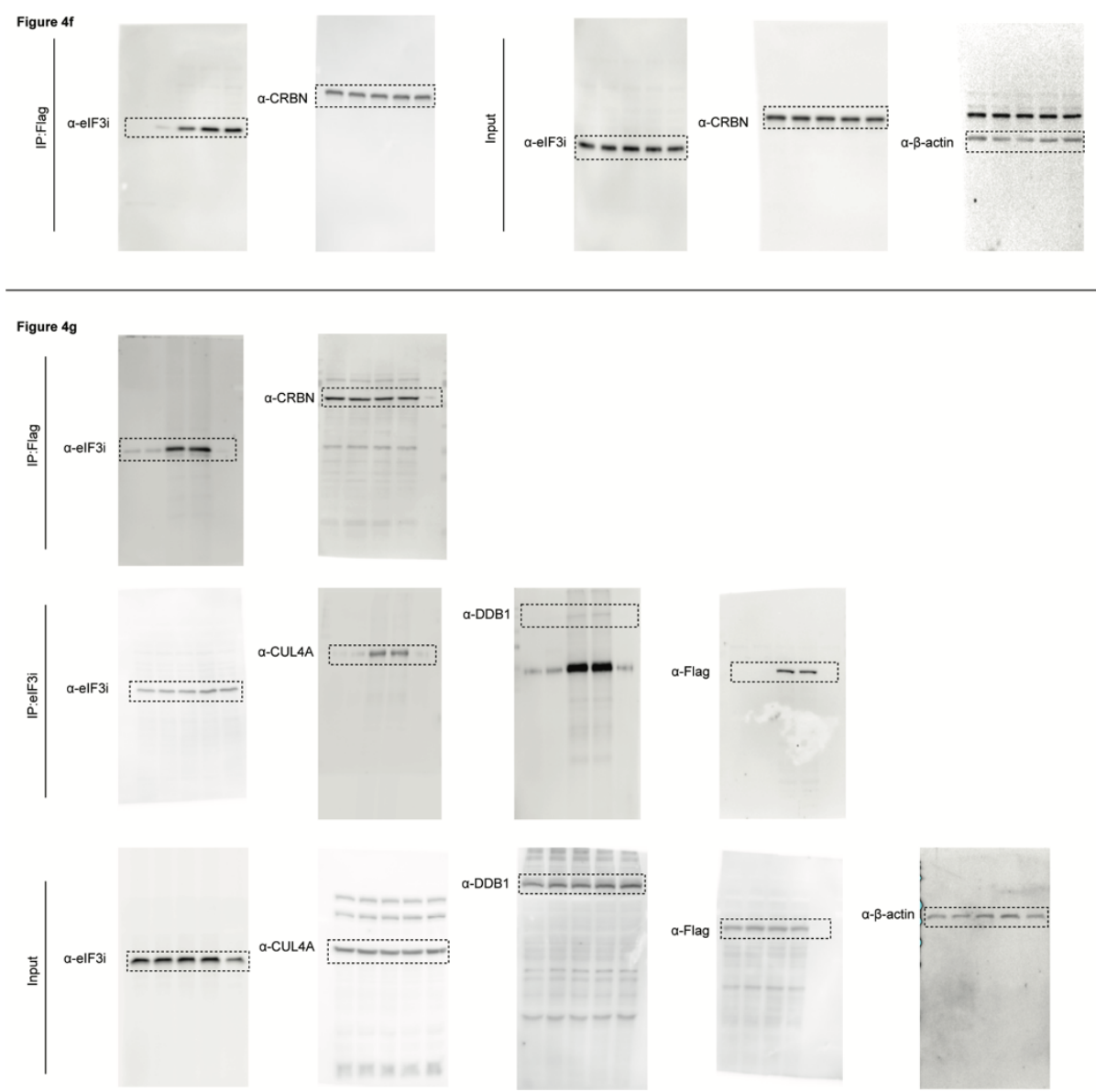

Figure S11 | Uncropped immunoblots related to Figure 4f and Figure $\mathbf{4 g}$. 

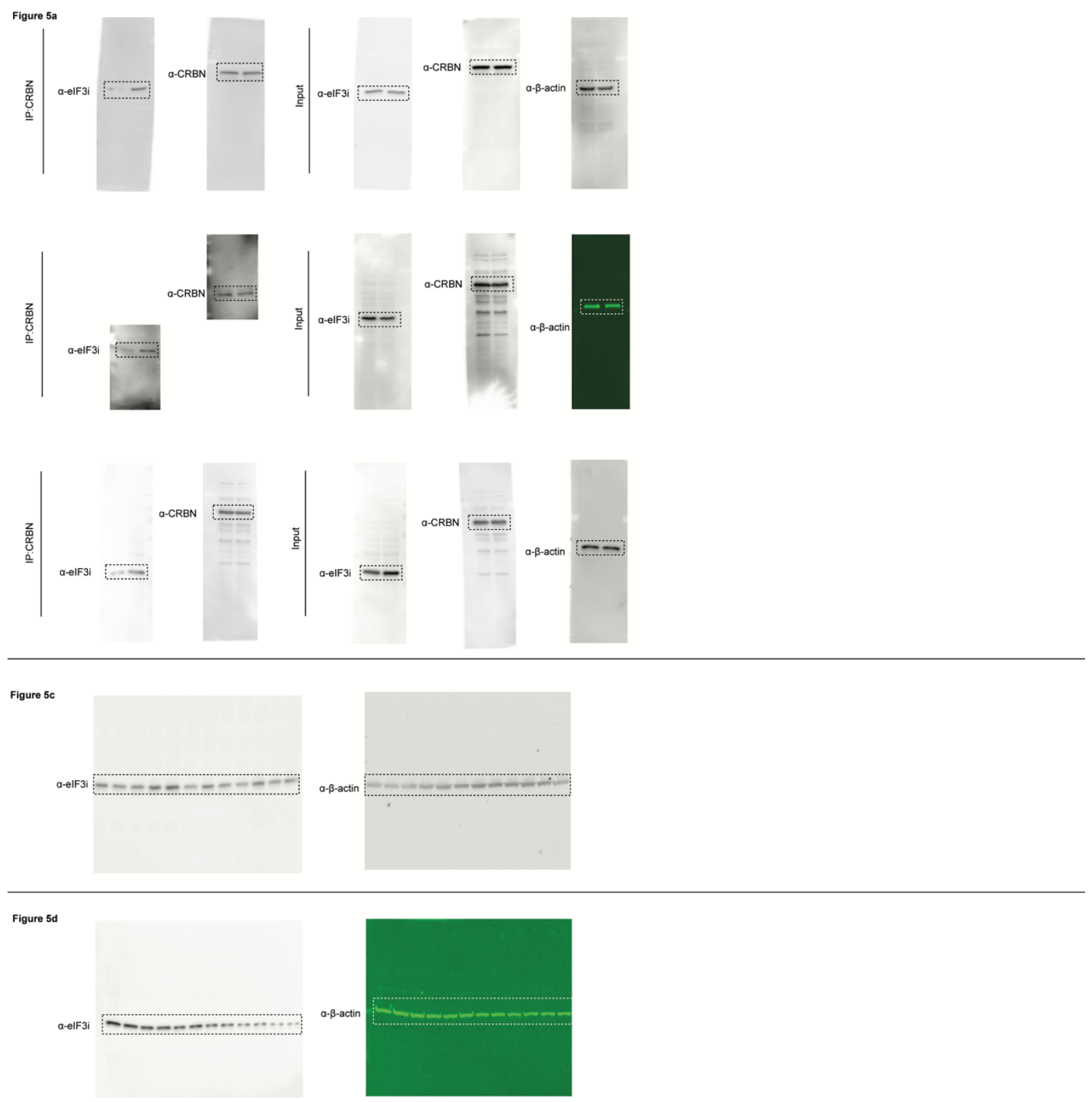

Figure S12 | Uncropped immunoblots related to Figure 5a, Figure 5c and Figure 5d. 


\section{Methods}

\section{General methods and instrumentation.}

Photoirradiation was performed using a Dymax ECE 5000 UV Light-Curing Flood Lamp system (Cat \# 41060) in a $4{ }^{\circ} \mathrm{C}$ cold room unless otherwise noted. The lamp was turned on for at least 15 minutes prior to use.

Protein quantification was performed by bicinchoninic acid assay on a multimode microplate reader FilterMax F3 (Molecular Devices LLC, Sunnyvale, CA). Sonication of cells or protein pellets was performed using a Branson SFX250 Sonifier (Branson Ultrasonics, Brookfield, CT). In-gel fluorescence were detected on an Azure Imager C600 (Azure Biosystems, Inc., Dublin, CA). AlphaScreen assay was performed on a multimode microplate reader SpectraMax i3x. DNA or RNA concentration was measured with a NanoDrop One UV-vis spectrophotometer (Thermo Fisher, Cat \# ND-ONE-W).

Proteomics experiments were performed on a Lumos Tribrid Orbitrap (Thermo Scientific) equipped with an UltiMate 3000 RSLCnano System (Thermo Scientific) within the Mass Spectrometry and Proteomics Resource Laboratory at Harvard University (MSPRL). Peptides were dried using an Eppendorf Vacufuge (Speed-Vac). Cell sorting experiments were performed on a BD FACSAria II cell sorter (BD Bioscience). Flow cytometry quantification were performed on BD LSR Fortessa (BD Bioscience, SORP) and BD LSR II flow cytometric analyzers (BD Biosciences, SORP).

For immunoblotting analysis, proteins were transferred from SDS-PAGE gels to nitrocellulose membranes using iBlot-2 dry blotting system (Thermo Scientific). Membranes were blocked with Tris buffered saline containing $0.1 \%$ Tween-20 and 5\% BSA and incubated with the primary antibodies and the secondary antibodies sequentially. Immunoblots images were captured by Azure Imager C600 (Azure Biosystems, Dublin, CA).

\section{General chemical methods.}

All reactions were performed in single-neck, oven-dried, round-bottomed flasks fitted with rubber septa under a positive pressure of argon, unless otherwise noted. Air- and moisturesensitive liquids were transferred via syringe or stainless-steel cannula. Organic solutions were concentrated by rotary evaporation at $30-33{ }^{\circ} \mathrm{C}$. Normal phase flash-column chromatography was performed as described by Still and co-workers. ${ }^{1}$ Normal phase purifications employ silica gel (60 A, 40-63 $\mu \mathrm{m}$ particle size) purchased from Silicycle (Quebec, Canada). Analytical thin-layer chromatography (TLC) was performed using glass plates precoated with silica gel $(0.25 \mathrm{~mm}, 60 \AA$ pore size $)$ containing a fluorescent indicator $(254 \mathrm{~nm})$. TLC plates were visualized by exposure to ultraviolet light (UV), iodine $\left(\mathrm{I}_{2}\right)$, and/or submersion in $p$-anisaldehyde or ninhydrin followed by brief heating with a heat gun (10-15 s).

Commercial solvents and reagents were used as received with the following exceptions. Lenalidomide was obtained from BioVision (Cat \# 1862-25). Minimalist tag was prepared according to the method of Yao and co-workers. ${ }^{2}$ The cleavable biotin azide probe was synthesized according to the method of Woo and co-workers ${ }^{3}$ and the cleavable biotin picolylazide probe was synthesized according to the method of Woo and co-workers. ${ }^{4}$ Cycloheximide $(\mathrm{CHX})$ was purchased from Sigma-Aldrich (Cat \# C4859). Dichloromethane, N,Ndimethylformamide and tetrahydrofuran were purified according to the method of Grubbs and co-workers. ${ }^{5}$ Triethylamine was distilled over calcium hydride under an atmosphere of argon.

\section{Chemical instrumentation.}

Proton nuclear magnetic resonance spectra $\left({ }^{1} \mathrm{H}\right.$ NMR) were recorded at $500 \mathrm{MHz}$ at $25{ }^{\circ} \mathrm{C}$ unless otherwise noted. Chemical shifts are expressed in parts per million (ppm, $\delta$ scale) 
downfield from tetramethylsilane and are referenced to residual protium in the NMR solvent $\left[\mathrm{CHCl}_{3}, \delta 7.26 ; \mathrm{CHD}_{2} \mathrm{OD}, \delta 3.31 ;\left(\mathrm{CHD}_{2}\right)\left(\mathrm{CD}_{3}\right) \mathrm{SO}, \delta\right.$ 2.49]. Data are represented as follows: chemical shift, multiplicity $(\mathrm{s}=$ singlet, $\mathrm{d}=$ doublet, $\mathrm{t}=$ triplet, $\mathrm{q}=$ quartet, quin $=$ quintet, $\mathrm{m}=$ multiplet and/or multiple resonances, $b r=$ broad, app = apparent), integration, coupling constant in Hertz, and assignment. Proton-decoupled carbon nuclear magnetic resonance spectra $\left({ }^{13} \mathrm{C}\right.$ NMR) were recorded at $125 \mathrm{MHz}$ at $25{ }^{\circ} \mathrm{C}$ unless otherwise noted. Chemical shifts are expressed in parts per million (ppm, $\delta$ scale) downfield from tetramethylsilane and are referenced to the carbon resonances of the solvent $\left[\mathrm{CDCl}_{3}, \delta 77.2 ; \mathrm{CD}_{3} \mathrm{OD}, \delta 49.0\right.$; $\left.\left(\mathrm{CD}_{3}\right)_{2} \mathrm{SO}, \delta 39.5\right] .{ }^{13} \mathrm{C}$ NMR and data are represented as follows: chemical shift, carbon type. Infrared (IR) spectra were obtained using a Shimadzu 8400S FT-IR spectrometer referenced to a polystyrene standard. Data are represented as follows: frequency of absorption $\left(\mathrm{cm}^{-1}\right)$, intensity of absorption ( $\mathrm{s}=$ strong, $\mathrm{m}=$ medium, $\mathrm{w}=$ weak, $\mathrm{br}=$ broad). Small molecule highresolution mass spectrometry (HRMS) measurements were obtained at the Harvard University Chemistry and Chemical Biology Department Mass Spectrometry Facility using an Agilent 1260 UPLC-MS Bruker connected in line with a microTOF-Q II hybrid quadrupole-time of flight MS or at the Harvard Center for Mass Spectrometry using a Thermo q-Exactive Plus. Preparative HPLC was performed on Waters Prep 150 LC System with XBridge BEH $\mathrm{C}_{18}$ OBD Prep Column, $130 \AA, 5 \mu \mathrm{m}, 19 \mathrm{~mm} \times 100 \mathrm{~mm}$. 


\section{Synthetic procedures.}

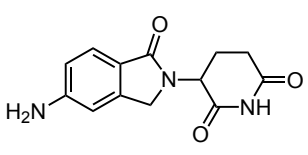

s1

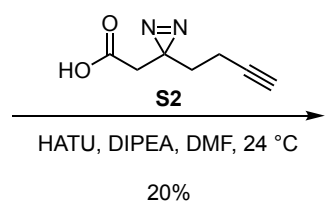

$20 \%$

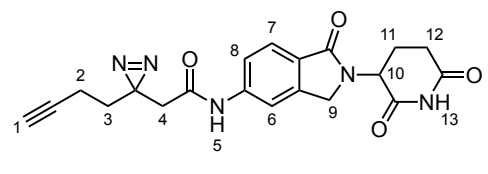

photo-lenalidomide

\section{Synthesis of photolenalidomide:}

The glutarimide $\mathbf{S} 1^{6}(19.2 \mathrm{mg}, 74.1 \mu \mathrm{mol}, 1.20$ equiv.) was added in a single portion to a stirred solution of the carboxylic acid $\mathbf{S 2}^{7}(9.4 \mathrm{mg}, 61.8 \mu \mathrm{mol}, 1.00$ equiv.). Sodium sulfate $(26.3 \mathrm{mg}$, $185 \mu \mathrm{mol}, 3.00$ equiv.), HATU (70.5 mg, $197 \mu \mathrm{mol}, 3.60$ equiv.) and $N, N$-diisopropylethylamine $(32.4 \mu \mathrm{L}, 185 \mu \mathrm{mol}, 3.60$ equiv.) were sequentially added to the mixture and the reaction mixture was stirred for $24 \mathrm{~h}$ at $25{ }^{\circ} \mathrm{C}$. The product mixture was diluted with ethyl acetate $(5.0$ $\mathrm{mL}$ ) and transferred to a separatory funnel. The diluted product mixture was washed with saturated aqueous sodium chloride $(5.0 \mathrm{~mL})$ and $10 \%$ aqueous citric acid $(5.0 \mathrm{~mL})$. The layers were mixed, and the aqueous layer was extracted by ethyl acetate $(3 \times 5.0 \mathrm{~mL})$. The combined organic layers were dried over sodium sulfate and concentrated to dryness. The crude product was purified by flash-column chromatography (eluting with $2 \%$ methanol-dichloromethane, grading to $4 \%$ methanol-dichloromethane, three steps). The obtained product mixture was further purified by HPLC (grading $95 \%$ to $5 \%$ water-acetonitrile over $25 \mathrm{~min}$, flow rate $=10$ $\mathrm{mL} / \mathrm{min}$, retention time $=12.3 \mathrm{~min}$ ) to afford the titled compound as a colorless solid (photolenalidomide, $4.8 \mathrm{mg}, 20 \%$ ).

$\mathrm{R}_{f}=0.25$ (4\% methanol-dichloromethane; UV). ${ }^{1} \mathrm{H}$ NMR $\left(500 \mathrm{MHz}\right.$, methanol- $\left.d_{4}\right): \delta 8.03$ (s, $\left.1 \mathrm{H}, \mathrm{H}_{6}\right), 7.74\left(\mathrm{~d}, J=8.3 \mathrm{~Hz}, 1 \mathrm{H}, \mathrm{H}_{7}\right), 7.52\left(\mathrm{dd}, J=8.3,1.9 \mathrm{~Hz}, 1 \mathrm{H}, \mathrm{H}_{8}\right), 5.14(\mathrm{dd}, J=13.4,5.2$ $\left.\mathrm{Hz}, 1 \mathrm{H}, \mathrm{H}_{10}\right), 4.51\left(\mathrm{~d}, J=17.0 \mathrm{~Hz}, 1 \mathrm{H}, \mathrm{H}_{9}\right), 4.45\left(\mathrm{~d}, J=17.0 \mathrm{~Hz}, 1 \mathrm{H}, \mathrm{H}_{9}\right), 2.91$ (ddd, $J=17.5$, 13.5, 5.3 Hz, 1H, $\mathrm{H}_{12}$ ), 2.78 (ddd, $J=17.5,4.5,2.4 \mathrm{~Hz}, 1 \mathrm{H}, \mathrm{H}_{12}$ ), 2.50 (dddd, $J=13.5,13.5$, 13.5, $\left.4.5 \mathrm{~Hz}, 2 \mathrm{H}, \mathrm{H}_{11}\right), 2.52\left(\mathrm{~s}, 2 \mathrm{H}, \mathrm{H}_{4}\right), 2.29\left(\mathrm{t}, J=2.6 \mathrm{~Hz}, 1 \mathrm{H}, \mathrm{H}_{1}\right), 2.17$ (dddd, $J=13.5,5.3$, 5.3, $\left.2.4 \mathrm{~Hz}, 1 \mathrm{H}, \mathrm{H}_{11}\right), 2.11\left(\mathrm{td}, J=7.4,2.6 \mathrm{~Hz}, 2 \mathrm{H}, \mathrm{H}_{2}\right), 1.79\left(\mathrm{t}, J=7.4 \mathrm{~Hz}, 2 \mathrm{H}, \mathrm{H}_{3}\right) .{ }^{13} \mathrm{C}$ NMR (126 MHz, methanol- $\left.d_{4}\right)$ : $\delta 173.3(\mathrm{C}), 170.8(\mathrm{C}), 169.7(\mathrm{C}), 167.9(\mathrm{C}), 143.6(\mathrm{C}), 142.2(\mathrm{C})$, $126.7(\mathrm{C}), 123.6(\mathrm{CH}), 119.3(\mathrm{CH}), 113.7(\mathrm{CH}), 82.1(\mathrm{C}), 69.0(\mathrm{CH}), 52.2(\mathrm{CH}), 47.9\left(\mathrm{CH}_{2}\right)$, $41.0\left(\mathrm{CH}_{2}\right), 32.2\left(\mathrm{CH}_{2}\right), 31.0\left(\mathrm{CH}_{2}\right), 25.9(\mathrm{C}), 22.7\left(\mathrm{CH}_{2}\right), 12.4\left(\mathrm{CH}_{2}\right)$. IR (ATR-FTIR), $\mathrm{cm}^{-1}$ : 3277 (br), 3087 (br), $1671(\mathrm{~s}), 1553(\mathrm{~m}), 1491(\mathrm{~m}), 1434(\mathrm{~m}), 1370(\mathrm{~m}), 1259(\mathrm{~m}), 1234(\mathrm{~m})$, $1198(\mathrm{~m}), 773(\mathrm{w}), 668(\mathrm{w})$. HRMS-ESI $(\mathrm{m} / \mathrm{z})$ : $[\mathrm{M}+\mathrm{H}]^{+}$calculated for $\mathrm{C}_{20} \mathrm{H}_{20} \mathrm{~N}_{5} \mathrm{O}_{4}, 394.1510$; found, 394.1512 . 


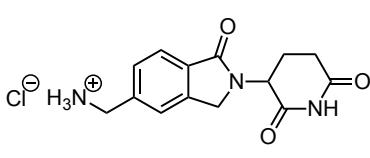

S3

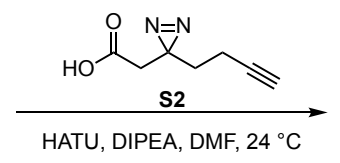

$44 \%$

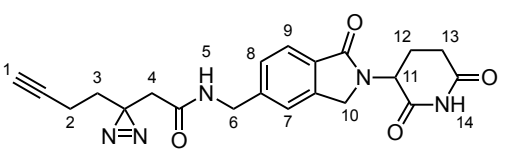

\section{Synthesis of analogue 1:}

The compound $\mathbf{S} 3^{8}(6.4 \mathrm{mg}, 20.8 \mu \mathrm{mol}, 1.00$ equiv.) was added in a single portion to a stirred solution of $\mathbf{S 2}$ (3.8 mg, $25.0 \mu \mathrm{mol}, 1.20$ equiv.) in $N, N$-dimethylformamide $(210 \mu \mathrm{L})$. HATU (11.9 mg, $31.2 \mu \mathrm{mol}, 1.50$ equiv.) and $N, N$-diisopropylethylamine $(10.9 \mu \mathrm{L}, 62.4 \mu \mathrm{mol}, 3.00$ equiv.) were added in sequence to the reaction mixture at $25^{\circ} \mathrm{C}$. The reaction mixture was stirred for $24 \mathrm{~h}$ at $25{ }^{\circ} \mathrm{C}$. The product mixture was diluted with ethyl acetate $(5.0 \mathrm{~mL})$ and transferred to a separatory funnel. The diluted product mixture was washed with saturated aqueous sodium chloride $(5.0 \mathrm{~mL})$ and $1 \mathrm{~N}$ hydrochloric acid $(5.0 \mathrm{~mL})$. The aqueous layers were combined, and the aqueous layer was extracted by chloroform ( $3 \times 5.0 \mathrm{~mL})$. The organic layers were combined, and the combined organic layers were dried over sodium sulfate. The dried solution was filtered, and the filtrate was concentrated to dryness. The crude product was purified by flash-column chromatography (eluting with $2 \%$ methanol-dichloromethane, grading to $4 \%$ methanol-dichloromethane, three steps). The obtained product mixture was further purified by HPLC (grading $95 \%$ to $5 \%$ water-acetonitrile over $25 \mathrm{~min}$, flow rate $=10$ $\mathrm{mL} / \mathrm{min}$, retention time $=10.8 \mathrm{~min})$, to afford the titled compound as a colorless solid $(\mathbf{1}, 4.4$ $\mathrm{mg}, 44 \%)$.

$\mathrm{R}_{f}=0.25$ (4\% methanol-dichloromethane; UV, anis aldehyde). ${ }^{1} \mathrm{H}$ NMR (400 MHz, dimethylsulfoxide- $\left.d_{6}\right): \delta 10.97\left(\mathrm{~s}, 1 \mathrm{H}, \mathrm{H}_{14}\right), 8.54\left(\mathrm{t}, J=6.0 \mathrm{~Hz}, 1 \mathrm{H}, \mathrm{H}_{5}\right), 7.68(\mathrm{~d}, J=7.8 \mathrm{~Hz}, 1 \mathrm{H}$, $\left.\mathrm{H}_{9}\right), 7.46\left(\mathrm{~s}, 1 \mathrm{H}, \mathrm{H}_{7}\right), 7.39$ (dd, $\left.J=7.8,1.4 \mathrm{~Hz}, 1 \mathrm{H}, \mathrm{H}_{8}\right), 5.10\left(\mathrm{dd}, J=13.2,5.2 \mathrm{~Hz}, 1 \mathrm{H}, \mathrm{H}_{5}\right)$, $4.44\left(\mathrm{~d}, J=17.3 \mathrm{~Hz}, 1 \mathrm{H}, \mathrm{H}_{10}\right), 4.40-4.34\left(\mathrm{~m}, 2 \mathrm{H}, \mathrm{H}_{6}\right), 4.31\left(\mathrm{~d}, J=17.3 \mathrm{~Hz}, 1 \mathrm{H}, \mathrm{H}_{10}\right), 2.91$ (ddd, $J=17.3,13.2,5.2 \mathrm{~Hz}, 1 \mathrm{H}, \mathrm{H}_{13}$ ), $2.82\left(\mathrm{t}, J=2.7 \mathrm{~Hz}, 1 \mathrm{H}, \mathrm{H}_{1}\right.$ ), 2.60 (ddd, $J=17.3,4.4,2.0$ $\mathrm{Hz}, 1 \mathrm{H}, \mathrm{H}_{13}$ ), 2.39 (dddd, $\left.J=13.2,13.2,13.2,4.4 \mathrm{~Hz}, 1 \mathrm{H}, \mathrm{H}_{12}\right), 2.31$ (s, 2H, $\mathrm{H}_{4}$ ), 2.04 (dt, $J=$ 7.5, $2.7 \mathrm{~Hz}, 2 \mathrm{H}, \mathrm{H}_{2}$ ), 2.00 (dddd, $J=13.2,5.2,5.2,2.0 \mathrm{~Hz}, 1 \mathrm{H}, \mathrm{H}_{12}$ ), 1.67 (t, J = 7.5 Hz, $2 \mathrm{H}$, $\left.\mathrm{H}_{3}\right) \cdot{ }^{13} \mathrm{C}$ NMR $(\mathrm{MHz}):,{ }^{13} \mathrm{C}$ NMR (126 MHz, dimethylsulfoxide- $\left.d_{6}\right) \delta 172.9(\mathrm{C}), 171.1(\mathrm{C}), 167.9$ $(\mathrm{C}), 167.4(\mathrm{C}), 143.5(\mathrm{C}), 142.4(\mathrm{C}), 130.5(\mathrm{C}), 127.1(\mathrm{CH}), 123.0(\mathrm{CH}), 122.1(\mathrm{CH}), 83.1(\mathrm{C})$, $71.8(\mathrm{CH}), 51.6(\mathrm{CH}), 47.1\left(\mathrm{CH}_{2}\right), 42.2\left(\mathrm{CH}_{2}\right), 40.0\left(\mathrm{CH}_{2}\right), 31.9\left(\mathrm{CH}_{2}\right), 31.2\left(\mathrm{CH}_{2}\right), 26.7(\mathrm{C})$, $22.5\left(\mathrm{CH}_{2}\right), 12.6\left(\mathrm{CH}_{2}\right)$. $\delta$. IR (ATR-FTIR), cm${ }^{-1}: 3258(\mathrm{br}), 3095(\mathrm{br}), 1707(\mathrm{~s}), 1400(\mathrm{~m}), 1263$ $(\mathrm{m}), 1178(\mathrm{~m}), 746(\mathrm{w}), 725(\mathrm{w})$. HRMS-ESI $(\mathrm{m} / \mathrm{z}):[\mathrm{M}+\mathrm{H}]^{+}$calculated for $\mathrm{C}_{21} \mathrm{H}_{21} \mathrm{~N}_{5} \mathrm{O}_{4}, 408.1666$; found, 408.1662 . 


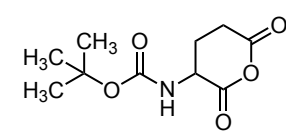

s4

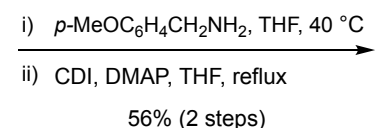

$56 \%$ (2 steps)

Synthesis of S5:

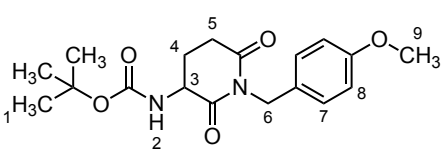

S5

\section{Step 1}

$\mathrm{N}$-Boc-glutamic acid anhydride (S4, $502 \mathrm{mg}, 2.19 \mathrm{mmol}, 1.00$ equiv.) and $p$-methoxybenzyl amine $\left(300 \mu \mathrm{L}, 2.30 \mathrm{mmol}, 1.05\right.$ equiv.) were dissolved in tetrahydrofuran $(3.64 \mathrm{~mL})$ at $25^{\circ} \mathrm{C}$. The resulting mixture was stirred for $24 \mathrm{~h}$ at $40^{\circ} \mathrm{C}$. The product mixture was cooled to $25^{\circ} \mathrm{C}$ and acidified to $\mathrm{pH} 1$ with $1 \mathrm{~N}$ hydrochloric acid. The acidic aqueous layer was extracted by ethyl acetate $(3 \times 10 \mathrm{~mL})$. The organic layers were combined, and the combined organic layers were dried over sodium sulfate and filtered. The filtrate was concentrated under vacuum and used directly in the following step.

\section{Step 2}

The reaction mixture obtained from the previous step was dissolved in tetrahydrofuran (21.9 $\mathrm{mL}$ ) and carbonyl diimidazole $(1.06 \mathrm{~g}, 6.57 \mathrm{mmol}, 3.00$ equiv.) and 4-dimethylaminopyridine ( $53.5 \mathrm{mg}, 438 \mu \mathrm{mol}, 0.20$ equiv.) were added in sequence at $25{ }^{\circ} \mathrm{C}$. The resulting mixture was heated at reflux for $24 \mathrm{~h}$. The product mixture was cooled down to $25^{\circ} \mathrm{C}$ and acidified to $\mathrm{pH} 1$ with $1 \mathrm{~N}$ hydrochloric acid. The acidic aqueous layer was extracted by ethyl acetate $(3 \times 20$ $\mathrm{mL}$ ). The organic layers were combined, and the combined organic layer were dried over sodium sulfate. The dried solution was filtered, and the filtrate was concentrated under vacuum. The crude mixture was purified by flash-column chromatography (eluting with $20 \%$ ethyl acetate-hexanes, grading to $40 \%$ ethyl acetate-hexanes, two steps) to afford the titled compound (S5, $428 \mathrm{mg}, 56 \%$ over 2 steps).

$\mathrm{R}_{f}=0.25$ (40\% ethyl acetate-hexane; ninhydrin). ${ }^{1} \mathrm{H}$ NMR (500 MHz, chloroform- $d$ ): $\delta 7.31$ (d, $\left.J=8.5 \mathrm{~Hz}, 2 \mathrm{H}, \mathrm{H}_{8}\right), 6.81\left(\mathrm{~d}, J=8.5 \mathrm{~Hz}, 2 \mathrm{H}, \mathrm{H}_{7}\right), 5.41\left(\mathrm{bs}, 1 \mathrm{H}, \mathrm{H}_{2}\right), 4.90(\mathrm{~d}, J=13.5 \mathrm{~Hz}, 1 \mathrm{H}$, $\left.\mathrm{H}_{6}\right), 4.86\left(\mathrm{~d}, J=13.5 \mathrm{~Hz}, 1 \mathrm{H}, \mathrm{H}_{6}\right), 4.30-4.22\left(\mathrm{~m}, 1 \mathrm{H}, \mathrm{H}_{3}\right), 3.77\left(\mathrm{~s}, 3 \mathrm{H}, \mathrm{H}_{9}\right), 2.86(\mathrm{ddd}, J=18.0$, 4.5, $2.0 \mathrm{~Hz}, 1 \mathrm{H}, \mathrm{H}_{5}$ ), 2.70 (ddd, $\left.J=18.0,13.5,5.5 \mathrm{~Hz}, 1 \mathrm{H}, \mathrm{H}_{5}\right), 2.51-2.43\left(\mathrm{~m}, 1 \mathrm{H}, \mathrm{H}_{4}\right), 1.77$ (dddd, $\left.J=13.5,13.5,13.5,4.5 \mathrm{~Hz}, 1 \mathrm{H}, \mathrm{H}_{4}\right), 1.45\left(\mathrm{~s}, 9 \mathrm{H}, \mathrm{H}_{1}\right) .{ }^{13} \mathrm{C} \mathrm{NMR}(125 \mathrm{MHz}$, chloroformd): $\delta 171.9(\mathrm{C}), 171.2(\mathrm{C}), 159.0(\mathrm{C}), 155.6(\mathrm{C}), 130.4(\mathrm{CH} \times 2), 129.1(\mathrm{C}), 113.8(\mathrm{CH} \times 2)$, $80.2(\mathrm{CH}), 55.2\left(\mathrm{CH}_{2}\right), 52.5(\mathrm{C}), 43.1\left(\mathrm{CH}_{2}\right), 31.8\left(\mathrm{CH}_{2}\right), 28.3\left(\mathrm{CH}_{3} \times 3\right), 24.7\left(\mathrm{CH}_{2}\right)$. IR (ATRFTIR), $\mathrm{cm}^{-1} 3369$ (br), 2975 (w), $1674(\mathrm{~m}), 1513(\mathrm{~m}), 1246$ (m), 1156 (s), 729 (m). HRMS-ESI $(\mathrm{m} / \mathrm{z}):[\mathrm{M}+\mathrm{H}]^{+}$calculated for $\mathrm{C}_{18} \mathrm{H}_{25} \mathrm{~N}_{2} \mathrm{O}_{5}, 349.1758$; found, 349.1759. 


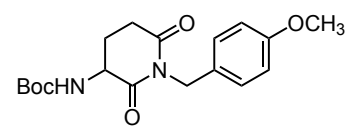

S5

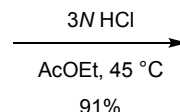

$91 \%$

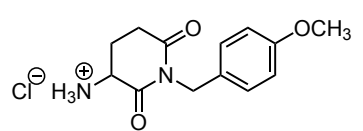

s6

Synthesis of S6:

$3 \mathrm{~N}$-Hydrochloric acid $(2.4 \mathrm{~mL}, 7.20 \mathrm{mmol}, 6.00$ equiv.) was added to a stirred solution of the compound S5 (418.5 mg, $1.20 \mathrm{mmol}, 1.00$ equiv.) in ethyl acetate $(6.0 \mathrm{~mL})$. The reaction mixture was stirred at $45^{\circ} \mathrm{C}$ for $24 \mathrm{~h}$. The product mixture was concentrated to dryness. The obtained residue was dissolved in benzene $(5.0 \mathrm{~mL})$ and concentrated to dryness to afford 1(4-methoxybenzyl)-2,6-dioxopiperidin-3-aminium chloride as a white solid (S6, $310 \mathrm{mg}, 91 \%$ ). The product was used directly in the following step. 


\section{Synthesis of S8:}

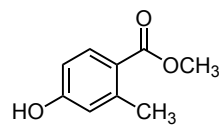

s7

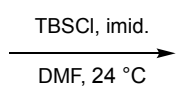

$90 \%$

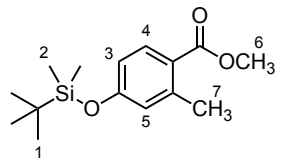

S8

Imidazole (3.04 g, $44.8 \mathrm{mmol}, 2.50$ equiv.) was added in a single portion to a stirred solution of the compound $\mathbf{S} 7^{9}$ (2.98 g, $17.9 \mathrm{mmol}, 1.00$ equiv.) in $N, N$-dimethylformamide $(9.9 \mathrm{~mL})$ at $25{ }^{\circ} \mathrm{C}$. The reaction mixture was cooled to $0{ }^{\circ} \mathrm{C}$ and tert-butyldimethylsilyl chloride $(2.97 \mathrm{~g}$, $19.7 \mathrm{mmol}, 1.1$ equiv.) was added in a single portion to the stirred mixture. The reaction mixture was warmed up to $25{ }^{\circ} \mathrm{C}$ and stirred for $24 \mathrm{~h}$. The product mixture was diluted with ethyl acetate $(20.0 \mathrm{~mL})$ and transferred to a separatory funnel. The diluted product mixture was washed with saturated aqueous sodium chloride $(20.0 \mathrm{~mL})$ and the aqueous layer was extracted into ethyl acetate $(20.0 \mathrm{~mL})$. The organic layers were combined, and the combined organic layers were dried over sodium sulfate. The dried solution was filtered, and the filtrate was concentrated to dryness. The crude product was purified by flash-column chromatography (eluting with $5 \%$ ethyl acetate-hexane) to afford the titled compound as a colorless oil (S8, $4.52 \mathrm{~g}, 90 \%)$.

$\mathrm{R}_{f}=0.25$ (2\% ethyl acetate-hexane; UV). ${ }^{1} \mathrm{H}$ NMR (500 MHz, chloroform- $d$ ): $\delta 7.86$ (d, $J=8.0$ $\left.\mathrm{Hz}, 1 \mathrm{H}, \mathrm{H}_{4}\right), 6.75-6.64\left(\mathrm{~m}, 2 \mathrm{H}, \mathrm{H}_{3}, \mathrm{H}_{5}\right), 3.85\left(\mathrm{~s}, 3 \mathrm{H}, \mathrm{H}_{6}\right), 2.56\left(\mathrm{~s}, 3 \mathrm{H}, \mathrm{H}_{7}\right), 0.98\left(\mathrm{~s}, 9 \mathrm{H}, \mathrm{H}_{1}\right)$, $0.22\left(\mathrm{~s}, 6 \mathrm{H}, \mathrm{H}_{2}\right) .{ }^{13} \mathrm{C}$ NMR (126 MHz, chloroform-d): $\delta 167.8(\mathrm{C}), 159.1(\mathrm{C}), 143.1(\mathrm{C}), 132.9$ $(\mathrm{CH}), 123.2(\mathrm{CH}), 122.5(\mathrm{C}), 117.3(\mathrm{CH}), 51.7\left(\mathrm{CH}_{3}\right), 25.8\left(\mathrm{CH}_{3} \times 3\right), 22.3,18.38,-4.21\left(\mathrm{CH}_{3}\right)$. IR (ATR-FTIR), $\mathrm{cm}^{-1} 2952(\mathrm{w}), 2930(\mathrm{w}), 2858(\mathrm{w}), 1719(\mathrm{~m}), 1600(\mathrm{~m}), 1246(\mathrm{~s}), 840(\mathrm{~m}), 808$ (m). HRMS-ESI $(m / z)$ : $[M+H]^{+}$calculated for $\mathrm{C}_{15} \mathrm{H}_{24} \mathrm{O}_{3} \mathrm{Si}$, 281.1567; found, 281.1568. 


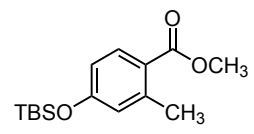

S8

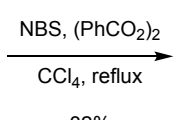

$32 \%$

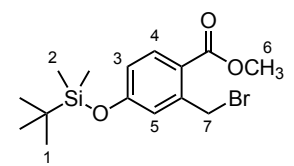

s9

\section{Synthesis of S9:}

$\mathrm{N}$-Bromosuccinimide (762 mg, $4.28 \mathrm{mmol}, 1.20$ equiv.) and benzoyl peroxide ( $86.4 \mathrm{mg}, 357$ $\mu \mathrm{mol}, 0.10$ equiv.) were added in sequence to a stirred solution of the compound $\mathbf{S 8}(1.00 \mathrm{~g}$, $3.57 \mathrm{mmol}, 1.00$ equiv.) in carbon tetrachloride $(12.0 \mathrm{~mL})$ at $25^{\circ} \mathrm{C}$. The reaction mixture was heated at reflux for $24 \mathrm{~h}$. The product mixture was cooled down to $25^{\circ} \mathrm{C}$ and filtrated through cotton. The filtrate was concentrated to dryness and purified by flash-column chromatography (eluting with $2 \%$ ethyl acetate-hexane) to afford the titled compound as a colorless oil (S9, $412 \mathrm{mg}, 32 \%)$.

$\mathrm{R}_{f}=0.25$ (2\% ethyl acetate-hexane; UV). ${ }^{1} \mathrm{H}$ NMR (500 MHz, chloroform- $d$ ) $\delta 7.91$ (d, $J=8.5$ $\left.\mathrm{Hz}, 1 \mathrm{H}, \mathrm{H}_{4}\right), 6.92\left(\mathrm{~d}, J=2.5 \mathrm{~Hz}, 1 \mathrm{H}, \mathrm{H}_{5}\right), 6.79\left(\mathrm{dd}, J=8.5,2.5 \mathrm{~Hz}, 1 \mathrm{H}, \mathrm{H}_{3}\right), 4.93\left(\mathrm{~s}, 2 \mathrm{H}, \mathrm{H}_{7}\right)$, 3.90 (s, 3H, $\left.\mathrm{H}_{6}\right), 0.99$ (s, 9H, $\left.\mathrm{H}_{1}\right), 0.24$ (s, 6H, H $\left.{ }_{2}\right){ }^{13} \mathrm{C}$ NMR (126 MHz, chloroform-d) $\delta 166.7$ $(\mathrm{C}), 159.5(\mathrm{C}), 141.8(\mathrm{C}), 133.7(\mathrm{CH}), 123.4(\mathrm{CH}), 121.7(\mathrm{C}), 119.9(\mathrm{CH}), 52.1\left(\mathrm{CH}_{3}\right), 31.8$ $\left(\mathrm{CH}_{2}\right), 25.7\left(\mathrm{CH}_{3} \times 3\right), 18.4(\mathrm{C}),-4.2\left(\mathrm{CH}_{3} \times 2\right)$. IR (ATR-FTIR), $\mathrm{cm}^{-1} 2954(\mathrm{w}), 2930(\mathrm{w}), 2858$ (w), $1719(\mathrm{~m}), 1601(\mathrm{~m}), 1307(\mathrm{~m}), 1275(\mathrm{~m}), 1253(\mathrm{~s}), 843(\mathrm{~m}), 783(\mathrm{~m})$. HRMS-ESI $(\mathrm{m} / \mathrm{z})$ : $[\mathrm{M}+\mathrm{H}]^{+}$calculated for $\mathrm{C}_{15} \mathrm{H}_{24} \mathrm{BrO}_{3} \mathrm{Si}, 359.0673$; found, 359.0671 . 


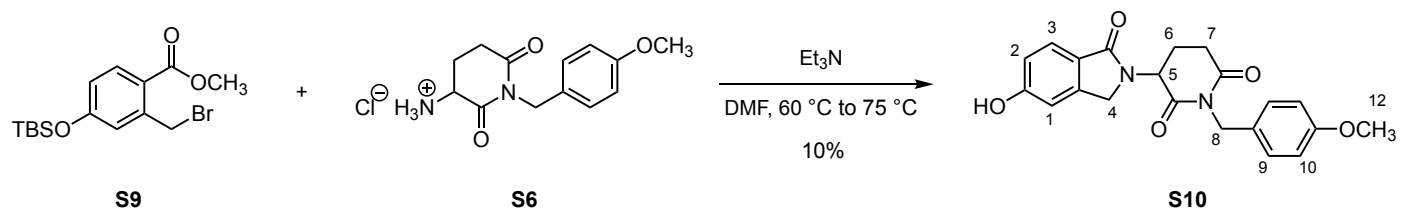

\section{Synthesis of S10:}

The compound $\mathbf{S} 6$ (100 mg, $351 \mu \mathrm{mol}, 1.00$ equiv.) and triethylamine $(147 \mu \mathrm{L}, 1.05 \mu \mathrm{mol}, 3.00$ equiv.) were added in sequence to the stirred solution of the compound S9 (126.2 $\mathrm{mg}, 351$ $\mu$ mol, 1.00 equiv.) in $N, N$-dimethylformamide $(1.8 \mathrm{~mL})$ at $25{ }^{\circ} \mathrm{C}$. The reaction mixture was transferred to an oil bath and stirred at $60{ }^{\circ} \mathrm{C}$ for $12 \mathrm{~h}$, and additionally stirred at $75{ }^{\circ} \mathrm{C}$ for $8 \mathrm{~h}$. The product mixture was cooled down to $25{ }^{\circ} \mathrm{C}$ and diluted with ethyl acetate $(5.0 \mathrm{~mL})$. The diluted mixture was transferred to a separatory funnel and washed with $1 \mathrm{~N}$-hydrochloric acid $(5.0 \mathrm{~mL})$. The aqueous layer was extracted into ethyl acetate $(5.0 \mathrm{~mL})$. The organic layers were combined, and the combined organic layers were dried over sodium sulfate. The dried solution was filtered, and the filtrate was concentrated to dryness. The residue obtained was purified by flash-column chromatography (grading from $60 \%$ ethyl acetate-hexane to $90 \%$ ethyl acetate-hexane over $30 \mathrm{~min}$ ) to afford the titled compound as a green solid (S10,13.1 $\mathrm{mg}, 10 \%)$.

$\mathrm{R}_{f}=0.33\left(90 \%\right.$ ethyl acetate-hexane; UV). ${ }^{1} \mathrm{H}$ NMR (500 MHz, chloroform- $d$ ): $\delta 7.65$ (d, $J=$ $\left.8.0 \mathrm{~Hz}, 1 \mathrm{H}, \mathrm{H}_{3}\right), 7.30\left(\mathrm{~d}, J=8.5 \mathrm{~Hz}, 2 \mathrm{H}, \mathrm{H}_{10}\right), 6.90\left(\mathrm{~d}, J=8.0 \mathrm{~Hz}, 1 \mathrm{H}, \mathrm{H}_{2}\right), 6.86\left(\mathrm{~s}, 1 \mathrm{H}, \mathrm{H}_{1}\right)$, $6.79\left(\mathrm{~d}, J=8.5 \mathrm{~Hz}, 2 \mathrm{H}, \mathrm{H}_{9}\right), 5.12\left(\mathrm{dd}, J=13.5,5.0 \mathrm{~Hz}, 1 \mathrm{H}, \mathrm{H}_{5}\right), 4.87\left(\mathrm{~s}, 2 \mathrm{H}, \mathrm{H}_{8}\right), 4.30(\mathrm{~d}, J=$ $\left.16.0 \mathrm{~Hz}, 1 \mathrm{H}, \mathrm{H}_{4}\right), 4.21\left(\mathrm{~d}, J=16.0 \mathrm{~Hz}, 1 \mathrm{H}, \mathrm{H}_{4}\right), 3.75\left(\mathrm{~s}, 3 \mathrm{H}, \mathrm{H}_{12}\right), 2.97$ (ddd, $J=17.5,4.5,2.5$ $\mathrm{Hz}, 1 \mathrm{H}, \mathrm{H}_{7}$ ), 2.82 (ddd, $J=17.5,13.5,5.5 \mathrm{~Hz}, 1 \mathrm{H}, \mathrm{H}_{7}$ ), 2.26 (dddd, $J=13.5,13.5,13.5,4.5$ $\left.\mathrm{Hz}, 1 \mathrm{H}, \mathrm{H}_{6}\right), 2.13$ (dddd, $\left.J=13.5,5.0,5.0,2.5 \mathrm{~Hz}, 1 \mathrm{H}, \mathrm{H}_{6}\right) \cdot{ }^{13} \mathrm{C} \mathrm{NMR}(126 \mathrm{MHz}$, chloroformd): $\delta 171.1$ (C), 170.3 (C), 170.1 (C), 160.7 (C), 159.2 (C), $144.2(\mathrm{C}), 130.7(\mathrm{CH} \times 2), 129.1$ (C), $125.8(\mathrm{CH}), 123.4(\mathrm{C}), 116.5(\mathrm{CH}), 114.0(\mathrm{CH} \times 2), 109.9(\mathrm{CH}), 55.4\left(\mathrm{CH}_{3}\right), 52.8(\mathrm{CH})$, $47.3\left(\mathrm{CH}_{2}\right), 43.4\left(\mathrm{CH}_{2}\right), 32.3\left(\mathrm{CH}_{2}\right), 22.8\left(\mathrm{CH}_{2}\right)$. IR (ATR-FTIR), $\mathrm{cm}^{-1} 2954(\mathrm{w}), 2930(\mathrm{w}), 2858$ $(\mathrm{w}), 1719(\mathrm{~m}), 1246(\mathrm{~s}), 840$ (s), 780 (s). HRMS-ESI $(\mathrm{m} / \mathrm{z})$ : $[\mathrm{M}+\mathrm{H}]^{+}$calculated for $\mathrm{C}_{21} \mathrm{H}_{21} \mathrm{~N}_{2} \mathrm{O}_{5}$, 381.1445; found, 381.1445 . 


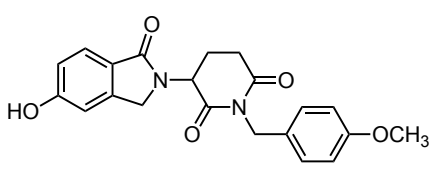

S10

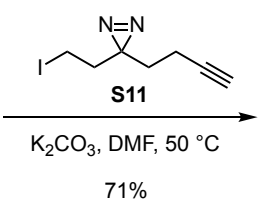

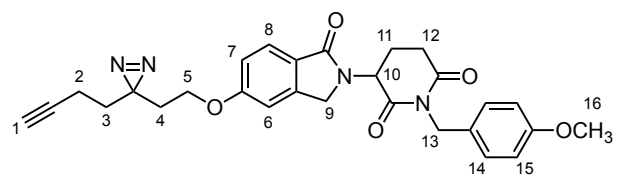

S12

\section{Synthesis of S12:}

Potassium carbonate $\left(9.0 \mathrm{mg}, 65.4 \mu \mathrm{mol}, 3.00\right.$ equiv.) and the compound $\mathbf{S} 11^{2}(14.8 \mathrm{mg}, 52.0$ $\mu \mathrm{mol}, 2.40$ equiv.) were added in sequence to a solution of the compound $\mathbf{S 1 0}(8.3 \mathrm{mg}, 21.8$ $\mu \mathrm{mol}, 1.00$ equiv.) in $\mathrm{N}, \mathrm{N}$-dimethylformamide $(220 \mu \mathrm{L})$ at $25{ }^{\circ} \mathrm{C}$. The reaction mixture was stirred for $48 \mathrm{~h}$ at $50{ }^{\circ} \mathrm{C}$. The product mixture was diluted with ethyl acetate $(5.0 \mathrm{~mL})$ and transferred to a separatory funnel. The diluted product mixture was washed with water (5.0 $\mathrm{mL})$ and the aqueous layer was extracted by ethyl acetate $(3 \times 5.0 \mathrm{~mL})$. The organic layers were combined, and the combined organic layers were dried over sodium sulfate. The dried solution was filtered, and the filtrate was concentrated to dryness. The crude product was purified by flash-column chromatography (eluting with $50 \%$ ethyl acetate-hexane) to afford the titled compound as a colorless solid $(\mathbf{S 1 2}, 7.7 \mathrm{mg}, 71 \%)$.

$\mathrm{R}_{f}=0.25$ (50\% ethyl acetate-hexane; UV). ${ }^{1} \mathrm{H}$ NMR $(500 \mathrm{MHz}$, chloroform- $d) \delta 7.79$ (d, $\mathrm{J}=$ $\left.8.5 \mathrm{~Hz}, 1 \mathrm{H}, \mathrm{H}_{8}\right), 7.33\left(\mathrm{~d}, J=8.5 \mathrm{~Hz}, 2 \mathrm{H}, \mathrm{H}_{14}\right), 6.99\left(\mathrm{dd}, J=8.5,2.0 \mathrm{~Hz}, 1 \mathrm{H}, \mathrm{H}_{7}\right), 6.92(\mathrm{~s}, 1 \mathrm{H}$, $\left.\mathrm{H}_{6}\right), 6.81\left(\mathrm{~d}, J=8.5 \mathrm{~Hz}, 2 \mathrm{H}, \mathrm{H}_{15}\right), 5.15\left(\mathrm{dd}, J=13.5,5.0 \mathrm{~Hz}, 1 \mathrm{H}, \mathrm{H}_{10}\right), 4.89\left(\mathrm{~s}, 2 \mathrm{H}, \mathrm{H}_{13}\right), 4.37$ (d, $\left.J=16.0 \mathrm{~Hz}, 1 \mathrm{H}, \mathrm{H}_{9}\right), 4.25$ (d, J=16.0 Hz, 1H, H9), 3.88 (t, J=6.0 Hz, 3H, H5), $3.78(\mathrm{~s}, 3 \mathrm{H}$, $\mathrm{H}_{16}$ ), 2.98 (ddd, $J=17.5,4.5,2.5 \mathrm{~Hz}, 1 \mathrm{H}, \mathrm{H}_{12}$ ), 2.84 (ddd, $J=17.5,13.5,5.5 \mathrm{~Hz}, 1 \mathrm{H}, \mathrm{H}_{12}$ ), 2.26 (dddd, $J=13.5,13.5,13.5,4.5 \mathrm{~Hz}, 1 \mathrm{H}, \mathrm{H}_{11}$ ), 2.14 (dddd, $J=13.5,5.5,5.5,2.5 \mathrm{~Hz}, 1 \mathrm{H}, \mathrm{H}_{11}$ ), $2.08\left(\mathrm{~d}, J=7.5,2.5 \mathrm{~Hz}, 2 \mathrm{H}, \mathrm{H}_{2}\right), 1.99\left(\mathrm{t}, J=2.5 \mathrm{~Hz}, 1 \mathrm{H}, \mathrm{H}_{1}\right), 1.94\left(\mathrm{t}, J=6.0 \mathrm{~Hz}, 2 \mathrm{H}, \mathrm{H}_{4}\right), 1.74$ (t, J = 7.5 Hz, 2H, H H $^{13}{ }^{13} \mathrm{C}$ NMR (126 MHz, chloroform-d) $\delta 171.1(\mathrm{C}), 170.2(\mathrm{C}), 169.3(\mathrm{C})$, $162.1(\mathrm{C}), 159.2(\mathrm{C}), 143.9(\mathrm{C}), 130.8(\mathrm{CH} \times 2), 129.3(\mathrm{C}), 125.7(\mathrm{CH}), 124.7(\mathrm{C}), 115.4(\mathrm{CH})$, $113.9(\mathrm{CH} x 2), 108.6(\mathrm{CH}), 82.8(\mathrm{C}), 69.4(\mathrm{CH}), 63.2\left(\mathrm{CH}_{2}\right), 55.4\left(\mathrm{CH}_{3}\right), 52.6(\mathrm{C}), 47.0\left(\mathrm{CH}_{2}\right)$, $43.3\left(\mathrm{CH}_{2}\right), 32.9\left(\mathrm{CH}_{2}\right), 32.8\left(\mathrm{CH}_{2}\right), 32.4\left(\mathrm{CH}_{2}\right), 26.7(\mathrm{C}), 22.9\left(\mathrm{CH}_{2}\right), 13.4\left(\mathrm{CH}_{2}\right)$. IR (ATRFTIR), $\mathrm{cm}^{-1} 2920(\mathrm{w}), 1729(\mathrm{~s}), 1230(\mathrm{~m}), 1141(\mathrm{~m})$. HRMS-ESI $(\mathrm{m} / \mathrm{z})$ : [M+H] ${ }^{+}$calculated for $\mathrm{C}_{28} \mathrm{H}_{29} \mathrm{~N}_{4} \mathrm{O}_{5}, 501.2132$; found, 501.2140 . 


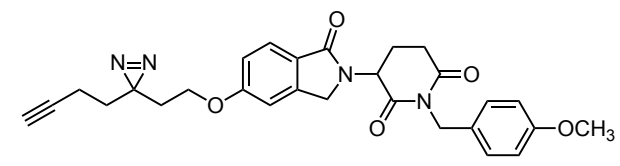

S12

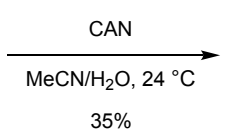

$35 \%$

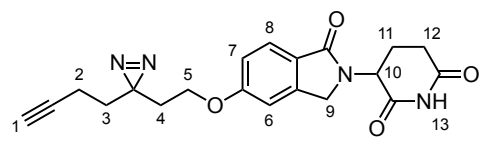

2

\section{Synthesis of analogue 2:}

Ammonium cerium(IV) nitrate (32.8 mg, $61.6 \mu \mathrm{mol}, 4.00$ equiv.) was added in a single portion to a solution of the compound $\mathbf{S} 12(7.7 \mathrm{mg}, 30.0 \mu \mathrm{mol}, 1.00$ equiv.) in acetonitrile $(170 \mu \mathrm{L})$ and water $(25.0 \mu \mathrm{L})$ at $25^{\circ} \mathrm{C}$. The reaction mixture was stirred for $6 \mathrm{~h}$ at $25{ }^{\circ} \mathrm{C}$. The product mixture was diluted with ethyl acetate $(5.0 \mathrm{~mL})$ and transferred to a separatory funnel. The diluted product mixture was washed with water $(5.0 \mathrm{~mL})$ and saturated aqueous sodium chloride $(5.0 \mathrm{~mL})$. The combined aqueous layers were extracted with chloroform $(3 \times 5.0 \mathrm{~mL})$. The organic layers were combined, and the combined organic layers were dried over sodium sulfate. The dried solution was filtered, and the filtrate was concentrated to dryness. The crude product was purified by flash-column chromatography (eluting with $1 \%$ methanoldichloromethane). The product mixture was further purified by recrystallization from dichloromethane to afford the titled compound as a colorless solid (2, $2.1 \mathrm{mg}, 35 \%)$.

$\mathrm{R}_{f}=0.25$ (2\% methanol-dichloromethane; UV). ${ }^{1} \mathrm{H}$ NMR (500 MHz, chloroform-d) $\delta 7.89$ (s, $\left.1 \mathrm{H}, \mathrm{H}_{13}\right), 7.80\left(\mathrm{~d}, J=8.4 \mathrm{~Hz}, 1 \mathrm{H}, \mathrm{H}_{8}\right), 7.00\left(\mathrm{dd}, J=8.4,2.2 \mathrm{~Hz}, 1 \mathrm{H}, \mathrm{H}_{7}\right), 6.94(\mathrm{~d}, J=2.2 \mathrm{~Hz}$, $\left.1 \mathrm{H}, \mathrm{H}_{16}\right), 5.21\left(\mathrm{dd}, J=13.2,5.2 \mathrm{~Hz}, 1 \mathrm{H}, \mathrm{H}_{10}\right), 4.45\left(\mathrm{~d}, J=15.8 \mathrm{~Hz}, 1 \mathrm{H}, \mathrm{H}_{9}\right), 4.29(\mathrm{~d}, J=15.8$ $\mathrm{Hz}, 1 \mathrm{H}, \mathrm{H}_{9}$ ), $3.88\left(\mathrm{t}, J=6.2 \mathrm{~Hz}, 2 \mathrm{H}, \mathrm{H}_{5}\right), 2.94-2.91\left(\mathrm{~m}, 1 \mathrm{H}, \mathrm{H}_{12}\right), 2.83$ (ddd, $J=17.9,13.2,5.2$ $\mathrm{Hz}, 1 \mathrm{H}, \mathrm{H}_{12}$ ), 2.35 (dddd, $J=13.2,13.2,13.2,4.8 \mathrm{~Hz}, 1 \mathrm{H}, \mathrm{H}_{11}$ ), 2.22 (dddd, $J=13.2,5.2,5.2$, $\left.2.2 \mathrm{~Hz}, 1 \mathrm{H}, \mathrm{H}_{11}\right), 2.08\left(\mathrm{td}, J=7.5,2.6 \mathrm{~Hz}, 2 \mathrm{H}, \mathrm{H}_{2}\right), 2.00\left(\mathrm{t}, J=2.6 \mathrm{~Hz}, 1 \mathrm{H}, \mathrm{H}_{1}\right), 1.95$ (t, $J=6.2$ $\left.\mathrm{Hz}, 2 \mathrm{H}, \mathrm{H}_{4}\right), 1.75\left(\mathrm{t}, J=7.5 \mathrm{~Hz}, 2 \mathrm{H}, \mathrm{H}_{3}\right) .{ }^{13} \mathrm{C}$ NMR $(126 \mathrm{MHz}$, chloroform-d) $\delta 171.1$ (C), 169.7 (C), $169.2(\mathrm{C}), 162.1(\mathrm{C}), 143.9(\mathrm{C}), 125.7(\mathrm{CH}), 124.5(\mathrm{C}), 115.5(\mathrm{CH}), 108.6(\mathrm{CH}), 82.8(\mathrm{C})$, $69.5(\mathrm{CH}), 63.2\left(\mathrm{CH}_{2}\right), 52.0(\mathrm{CH}), 47.0\left(\mathrm{CH}_{2}\right), 32.9\left(\mathrm{CH}_{2}\right), 32.8\left(\mathrm{CH}_{2}\right), 31.7\left(\mathrm{CH}_{2}\right), 26.7(\mathrm{C})$, $23.6\left(\mathrm{CH}_{2}\right), 13.4\left(\mathrm{CH}_{2}\right)$. IR (ATR-FTIR), 3285 (br), $3100(\mathrm{br}), 2924(\mathrm{br}), 1682(\mathrm{~s}), 1614$ (s), $1454(\mathrm{~m}), 1368(\mathrm{~m}), 1258$ (s), 1233 (s), 1199 (s), 1178 (s), 770 (w), 687 (w) cm ${ }^{-1}$. HRMS-ESI $(\mathrm{m} / \mathrm{z}):[\mathrm{M}+\mathrm{H}]^{+}$calculated for $\mathrm{C}_{20} \mathrm{H}_{21} \mathrm{~N}_{4} \mathrm{O}_{4}, 381.1557$; found, 381.1560 . 

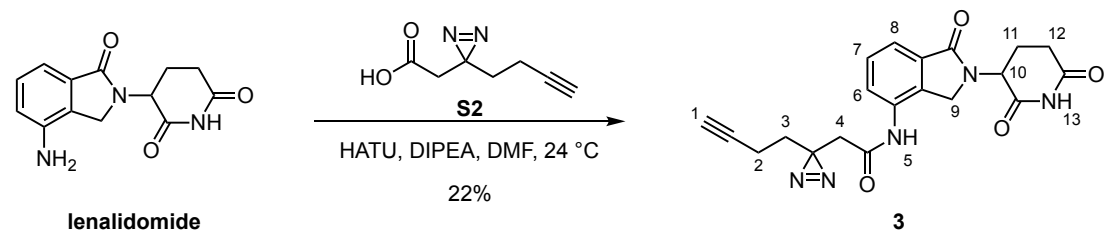

\section{Synthesis of analogue 3:}

Lenalidomide (14.2 mg, $54.8 \mu \mathrm{mol}, 1.00$ equiv.), sodium sulfate $(23.3 \mathrm{mg}, 164 \mu \mathrm{mol}, 3.00$ equiv.), HATU (75.0 mg, $197 \mu \mathrm{mol}, 3.60$ equiv.) and $N, N$-diisopropylethylamine $(26.0 \mu \mathrm{L}, 197$ $\mu \mathrm{mol}, 3.60$ equiv.) were added in a single portion to a stirred solution of the carboxylic acid $\mathbf{S 2}$ $\left(10.0 \mathrm{mg}, 65.7 \mu \mathrm{mol}, 1.20\right.$ equiv.) sequentially at $25^{\circ} \mathrm{C}$. The reaction mixture was stirred for $24 \mathrm{~h}$ at $25{ }^{\circ} \mathrm{C}$. The product mixture was diluted with ethyl acetate $(5.0 \mathrm{~mL})$ and transferred to a separatory funnel. The diluted product mixture was washed with saturated aqueous sodium chloride $(5.0 \mathrm{~mL})$ and $10 \%$ aqueous citric acid $(5.0 \mathrm{~mL})$. The layers were mixed, and the aqueous layer was extracted by ethyl acetate $(3 \times 5 \mathrm{~mL})$. The combined organic layers were dried over sodium sulfate and concentrated to dryness. The crude product was purified by flash-column chromatography (eluting with $2 \%$ methanol-dichloromethane, grading to $4 \%$ methanol-dichloromethane, three steps). The obtained product mixture was further purified by HPLC (grading $95 \%$ to $5 \%$ water-acetonitrile over $25 \mathrm{~min}$, flow rate $=10 \mathrm{~mL} / \mathrm{min}$, retention time $=11.8 \mathrm{~min})$, to afford the titled compound as a white solid $(3,4.6 \mathrm{mg}, 22 \%)$.

$\mathrm{R}_{f}=0.25$ (4\% methanol-dichloromethane; UV). ${ }^{1} \mathrm{H}$ NMR $\left(500 \mathrm{MHz}, \mathrm{DMSO}-d_{6}\right) \delta 11.03(\mathrm{~s}, 1 \mathrm{H}$, $\left.\mathrm{H}_{13}\right), 9.90\left(\mathrm{~s}, 1 \mathrm{H}, \mathrm{H}_{5}\right), 7.79$ (dd, J = 7.6, $\left.1.4 \mathrm{~Hz}, 1 \mathrm{H}, \mathrm{H}_{6} / \mathrm{H}_{8}\right), 7.57-7.47\left(\mathrm{~m}, 2 \mathrm{H}, \mathrm{H}_{6} / \mathrm{H}_{8}, \mathrm{H}_{7}\right), 5.15$ (dd, $\left.J=13.5,5.0 \mathrm{~Hz}, 1 \mathrm{H}, \mathrm{H}_{10}\right), 4.37\left(\mathrm{~d}, J=17.5 \mathrm{~Hz}, 1 \mathrm{H}, \mathrm{H}_{9}\right), 4.31\left(\mathrm{~d}, J=17.5 \mathrm{~Hz}, 1 \mathrm{H}, \mathrm{H}_{9}\right)$, $2.92\left(\mathrm{ddd}, J=17.4,13.5,5.0 \mathrm{~Hz}, 1 \mathrm{H}, \mathrm{H}_{12}\right), 2.85\left(\mathrm{t}, J=2.6 \mathrm{~Hz}, 1 \mathrm{H}, \mathrm{H}_{1}\right), 2.61$ (ddd, $J=17.4$, 5.0, $2.2 \mathrm{~Hz}, 1 \mathrm{H}, \mathrm{H}_{12}$ ), $2.54\left(\mathrm{~s}, 2 \mathrm{H}, \mathrm{H}_{4}\right), 2.34$ (dddd, $\left.J=13.5,13.5,13.5,4.6 \mathrm{~Hz}, 1 \mathrm{H}, \mathrm{H}_{11}\right), 2.11$ $-1.99\left(\mathrm{~m}, 3 \mathrm{H}, \mathrm{H}_{11}, \mathrm{H}_{2}\right), 1.73\left(\mathrm{t}, J=7.4 \mathrm{~Hz}, 2 \mathrm{H}, \mathrm{H}_{3}\right) .{ }^{13} \mathrm{C}$ NMR $\left(126 \mathrm{MHz}\right.$, DMSO- $\left.d_{6}\right): \delta$. IR (ATRFTIR), $\mathrm{cm}^{-1} 3272(\mathrm{br}), 2920(\mathrm{~m}), 2851(\mathrm{~m}), 1686(\mathrm{~s}), 1677(\mathrm{~s}), 1432(\mathrm{~m}), 1418(\mathrm{~m}), 1235(\mathrm{~m})$, $1204(\mathrm{~m}), 750(\mathrm{~m})$. HRMS-ESI $(\mathrm{m} / \mathrm{z})$ : $[\mathrm{M}+\mathrm{H}]^{+}$calculated for $\mathrm{C}_{20} \mathrm{H}_{20} \mathrm{~N}_{5} \mathrm{O}_{4}$ 394.1510; found, 394.1505. 


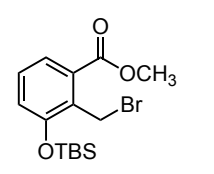

S13

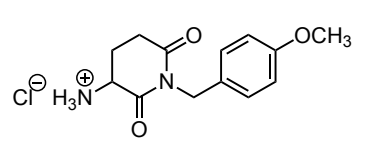

S6

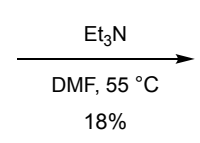

$18 \%$

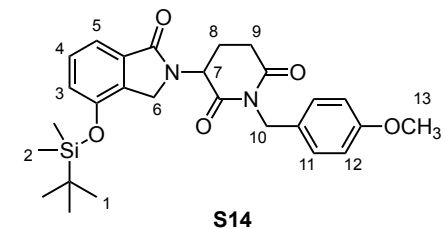

\section{Synthesis of S14:}

The compound $\mathbf{S 6}$ (159 mg, $557 \mu \mathrm{mol}, 1.00$ equiv.) and triethylamine (232 $\mu \mathrm{L}, 1.67 \mathrm{mmol}$, 3.00 equiv.) were added in sequence to a stirred solution of the compound $\mathbf{S} 13^{10}(200 \mathrm{mg}$, $557 \mu \mathrm{mol}, 1.00$ equiv.) in $\mathrm{N}, \mathrm{N}$-dimethylformamide $(2.8 \mathrm{~mL})$ at $25^{\circ} \mathrm{C}$. The reaction mixture was stirred for $24 \mathrm{~h}$ at $55{ }^{\circ} \mathrm{C}$. The product mixture was diluted with ethyl acetate $(10.0 \mathrm{~mL})$ and transferred to a separatory funnel. The diluted product mixture was washed with $1 \mathrm{~N}$ hydrochloric acid $(5.0 \mathrm{~mL})$ and the aqueous layer was extracted by ethyl acetate $(3 \times 5.0 \mathrm{~mL})$. The organic layers were combined, and the combined organic layers were dried over sodium sulfate. The dried solution was filtered, and the filtrate was concentrated to dryness. The crude product was purified by flash-column chromatography (eluting with $50 \%$ ethyl acetatehexane) to afford the titled compound as a white solid (S14, $50.3 \mathrm{mg}, 18 \%)$.

$\mathrm{R}_{f}=0.25$ (40\% ethyl acetate-hexane; UV). ${ }^{1} \mathrm{H}$ NMR (500 MHz, chloroform- $d$ ): $\delta 7.49$ (dd, $\mathrm{J}=$ 8.0, 0.5 Hz, 1H, $\left.\mathrm{H}_{5}\right), 7.39-7.30\left(\mathrm{~m}, 3 \mathrm{H}, \mathrm{H}_{4}, \mathrm{H}_{11}\right), 6.96\left(\mathrm{dd}, J=8.0,0.5 \mathrm{~Hz}, 1 \mathrm{H}, \mathrm{H}_{3}\right), 6.81(\mathrm{~d}, J$ $\left.=8.5 \mathrm{~Hz}, 2 \mathrm{H}, \mathrm{H}_{12}\right), 5.17\left(\mathrm{dd}, J=13.5,5.0 \mathrm{~Hz}, 1 \mathrm{H}, \mathrm{H}_{7}\right), 4.93\left(\mathrm{~d}, J=14.0 \mathrm{~Hz}, 1 \mathrm{H}, \mathrm{H}_{10}\right), 4.89(\mathrm{~d}$, $\left.J=14.0 \mathrm{~Hz}, 1 \mathrm{H}, \mathrm{H}_{10}\right), 4.31\left(\mathrm{~d}, J=16.0 \mathrm{~Hz}, 1 \mathrm{H}, \mathrm{H}_{6}\right), 4.21\left(\mathrm{~d}, J=16.0 \mathrm{~Hz}, 1 \mathrm{H}, \mathrm{H}_{6}\right), 3.78(\mathrm{~s}, 3 \mathrm{H}$, $\mathrm{H}_{13}$ ), 2.98 (ddd, $J=17.5,4.5,2.5 \mathrm{~Hz}, 1 \mathrm{H}, \mathrm{H}_{9}$ ), 2.84 (ddd, $J=17.5,13.5,5.0,1 \mathrm{H}, \mathrm{H}_{9}$ ), 2.33 (dddd, $J=13.5,13.5,13.5,5.0 \mathrm{~Hz}, 1 \mathrm{H}, \mathrm{H}_{8}$ ), 2.15 (dddd, $J=13.5,5.0,5.0,2.5 \mathrm{~Hz}, 1 \mathrm{H}, \mathrm{H}_{8}$ ), $1.00\left(\mathrm{~s}, 9 \mathrm{H}, \mathrm{H}_{1}\right), 0.26\left(\mathrm{~s}, 6 \mathrm{H}, \mathrm{H}_{2}\right) .{ }^{13} \mathrm{C}$ NMR $(126 \mathrm{MHz}$, chloroform-d): $\delta 171.1(\mathrm{C}), 170.1(\mathrm{C})$, $169.6(\mathrm{C}), 159.2(\mathrm{C}), 151.0(\mathrm{C}), 133.7(\mathrm{C}), 132.1(\mathrm{C}), 130.8(\mathrm{CH} \times 2), 129.8(\mathrm{CH}), 129.3(\mathrm{C})$, $121.7(\mathrm{CH}), 117.1(\mathrm{CH}), 113.9(\mathrm{CH} x), 55.4\left(\mathrm{CH}_{3}\right), 52.7(\mathrm{CH}), 45.3\left(\mathrm{CH}_{2}\right), 43.3\left(\mathrm{CH}_{2}\right), 32.4$ $\left(\mathrm{CH}_{2}\right), 25.8\left(\mathrm{CH}_{3} \times 3\right), 22.8\left(\mathrm{CH}_{2}\right), 18.3(\mathrm{C}),-4.06\left(\mathrm{CH}_{3}\right),-4.09\left(\mathrm{CH}_{3}\right)$. IR (ATR-FTIR), cm ${ }^{-1} 2054$ $(\mathrm{w}), 2930(\mathrm{w}), 1678(\mathrm{~s}), 1288(\mathrm{~m}), 1248(\mathrm{~m}), 831(\mathrm{~m})$. HRMS-ESI $(\mathrm{m} / \mathrm{z}):[\mathrm{M}+\mathrm{H}]^{+}$calculated for $\mathrm{C}_{27} \mathrm{H}_{35} \mathrm{~N}_{2} \mathrm{O}_{5} \mathrm{Si}$, 495.2310; found, 495.2315 . 

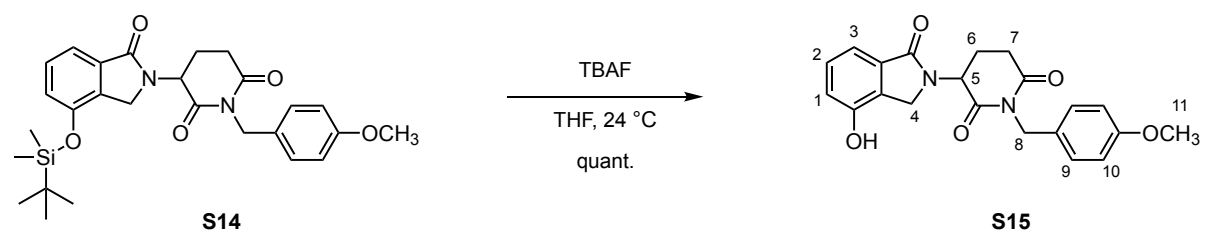

\section{Synthesis of S15:}

Tetrabutylammonium fluoride solution in tetrahydrofuran $(1.0 \mathrm{M}, 153 \mu \mathrm{L}, 153 \mathrm{mmol}, 1.50$ equiv.) was added dropwise to a stirred solution of the compound $\mathbf{S 1 4}(50.3 \mathrm{mg}, 102 \mu \mathrm{mol}$, 1.00 equiv.) in tetrahydrofuran $(510 \mu \mathrm{L})$ over $5 \mathrm{~min}$ at $0{ }^{\circ} \mathrm{C}$. The reaction mixture was stirred for $1 \mathrm{~h}$ at $25{ }^{\circ} \mathrm{C}$. The product mixture was diluted with ethyl acetate $(5.0 \mathrm{~mL})$ and saturated aqueous ammonium chloride solution $(5.0 \mathrm{~mL})$. The biphasic product mixture was transferred to a separatory funnel and the layers were separated. The aqueous layer was extracted by ethyl acetate $(3 \times 5 \mathrm{~mL})$. The organic layers were combined, and the combined organic layers were dried over sodium sulfate. The dried solution was filtered, and the filtrate was concentrated to dryness. The crude product was purified by flash-column chromatography (eluting with $60 \%$ ethyl acetate-hexane, grading to $80 \%$ ethyl acetate-hexane, three steps) to afford the titled compound as a colorless solid (S15, $38.6 \mathrm{mg}$, quantitative yield).

$\mathrm{R}_{f}=0.25\left(70 \%\right.$ ethyl acetate-hexane; UV). ${ }^{1} \mathrm{H}$ NMR $(500 \mathrm{MHz}$, chloroform- $d) \delta 7.43(\mathrm{~d}, J=$ 8.0, $\left.1 \mathrm{H}, \mathrm{H}_{3}\right), 7.32\left(\mathrm{~d}, J=8.5,2 \mathrm{H}, \mathrm{H}_{9}\right), 7.29\left(\mathrm{dd}, J=8.0,1 \mathrm{H}, \mathrm{H}_{2}\right), 6.95\left(\mathrm{~d}, J=8.0 \mathrm{~Hz}, 1 \mathrm{H}, \mathrm{H}_{1}\right)$, $6.81\left(\mathrm{~d}, J=8.5 \mathrm{~Hz}, 2 \mathrm{H}, \mathrm{H}_{10}\right), 5.16\left(\mathrm{dd}, J=13.5,5.0 \mathrm{~Hz}, 1 \mathrm{H}, \mathrm{H}_{5}\right), 4.89\left(\mathrm{~s}, 2 \mathrm{H}, \mathrm{H}_{8}\right), 4.38(\mathrm{~d}, J=$ $\left.16.0 \mathrm{~Hz}, 1 \mathrm{H}, \mathrm{H}_{4}\right), 4.28\left(\mathrm{~d}, J=16.0 \mathrm{~Hz}, 1 \mathrm{H}, \mathrm{H}_{4}\right), 3.77\left(\mathrm{~s}, 3 \mathrm{H}, \mathrm{H}_{11}\right), 2.99$ (ddd, $J=18.0,4.5,2.5$ $\mathrm{Hz}, 1 \mathrm{H}, \mathrm{H}_{7}$ ), 2.84 (ddd, $J=18.0,13.5,5.5 \mathrm{~Hz}, 1 \mathrm{H}, \mathrm{H}_{7}$ ), 2.29 (dddd, $J=13.5,13.5,13.5,4.5$ $\left.\mathrm{Hz}, 1 \mathrm{H}, \mathrm{H}_{6}\right), 2.15$ (dddd, $\left.J=13.5,13.5,5.5,2.5 \mathrm{~Hz}, 1 \mathrm{H}, \mathrm{H}_{7}\right) .{ }^{13} \mathrm{C}$ NMR $(126 \mathrm{MHz}$, chloroformd) $\delta 171.1(\mathrm{C}), 170.2(\mathrm{C}), 169.8(\mathrm{C}), 159.2(\mathrm{C}), 151.3(\mathrm{C}), 133.5(\mathrm{C}), 130.7(\mathrm{CH}$ x2), 130.0 $(\mathrm{CH}), 129.2(\mathrm{C}), 128.0(\mathrm{C}), 118.6(\mathrm{CH}), 116.4(\mathrm{CH}), 114.0(\mathrm{CH} x), 55.4\left(\mathrm{CH}_{3}\right), 52.9(\mathrm{CH})$, $45.3\left(\mathrm{CH}_{2}\right), 43.4\left(\mathrm{CH}_{2}\right), 32.4\left(\mathrm{CH}_{2}\right), 22.8\left(\mathrm{CH}_{2}\right)$. IR (ATR-FTIR), $\mathrm{cm}^{-1} 3171(\mathrm{br}), 2958(\mathrm{w}), 2924$ (w), 2853 (w), $1678(\mathrm{~s}), 1608(\mathrm{~m}), 1513(\mathrm{~m}), 1291(\mathrm{~m}), 1248(\mathrm{~m}), 1164(\mathrm{~m}), 733(\mathrm{~m})$. HRMSESI $(m / z):[\mathrm{M}+\mathrm{H}]^{+}$calculated for $\mathrm{C}_{21} \mathrm{H}_{21} \mathrm{~N}_{2} \mathrm{O}_{5}, 381.1445$; found, 381.1442 . 

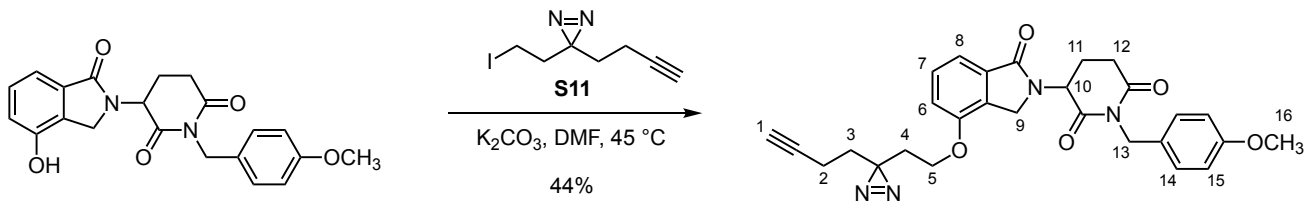

S15

S16

\section{Synthesis of S16:}

Potassium carbonate (42.1 mg, $304 \mu \mathrm{mol}, 3.00$ equiv.) and minimalist iodide $\mathbf{S 1 1 ^ { 2 }}(25.7 \mathrm{mg}$, $101 \mu \mathrm{mol}, 1.00$ equiv.) were added in sequence to a solution of the compound $\mathbf{S} 15$ (38.6 mg, $101 \mu \mathrm{mol}, 1.00$ equiv.) in $\mathrm{N}, \mathrm{N}$-dimethylformamide $(500 \mu \mathrm{L})$ at $25^{\circ} \mathrm{C}$. The reaction mixture was stirred for $24 \mathrm{~h}$ at $45{ }^{\circ} \mathrm{C}$. The product mixture was diluted with ethyl acetate $(10.0 \mathrm{~mL})$ and transferred to a separatory funnel. The diluted product mixture was washed with water (5.0 $\mathrm{mL})$ and the aqueous layer was extracted by ethyl acetate $(3 \times 5.0 \mathrm{~mL})$. The organic layers were combined, and the combined organic layers were dried over sodium sulfate. The dried solution was filtered, and the filtrate was concentrated to dryness. The crude product was purified by flash-column chromatography (eluting with $50 \%$ ethyl acetate-hexane, grading to $60 \%$ ethyl acetate-hexane, one step) to afford the titled compound as a colorless solid (S16, $16.8 \mathrm{mg}, 44 \%)$.

$\mathrm{R}_{f}=0.25\left(60 \%\right.$ ethyl acetate-hexane; UV). ${ }^{1} \mathrm{H}$ NMR $(500 \mathrm{MHz}$, chloroform- $d): \delta 7.50(\mathrm{~d}, \mathrm{~J}=$ $\left.8.0 \mathrm{~Hz}, 1 \mathrm{H}, \mathrm{H}_{6} / \mathrm{H}_{8}\right), 7.42\left(\mathrm{t}, J=8.0 \mathrm{~Hz}, 1 \mathrm{H}, \mathrm{H}_{7}\right), 7.34\left(\mathrm{~d}, J=8.5 \mathrm{~Hz}, 2 \mathrm{H}, \mathrm{H}_{14}\right), 6.97(\mathrm{~d}, J=8.0$ $\left.\mathrm{Hz}, 1 \mathrm{H}, \mathrm{H}_{6} / \mathrm{H}_{8}\right), 6.81\left(\mathrm{~d}, J=8.5 \mathrm{~Hz}, 2 \mathrm{H}, \mathrm{H}_{15}\right), 5.18\left(\mathrm{dd}, J=13.5,5.0 \mathrm{~Hz}, 1 \mathrm{H}, \mathrm{H}_{10}\right), 4.90(\mathrm{~s}, 2 \mathrm{H}$, $\left.\mathrm{H}_{13}\right), 4.41\left(\mathrm{~d}, J=16.5 \mathrm{~Hz}, 1 \mathrm{H}, \mathrm{H}_{9}\right), 4.32\left(\mathrm{~d}, J=16.5 \mathrm{~Hz}, 1 \mathrm{H}, \mathrm{H}_{9}\right), 3.98-3.94\left(\mathrm{~m}, 2 \mathrm{H}, \mathrm{H}_{5}\right), 3.77$ (s, 3H, $\mathrm{H}_{16}$ ), 2.99 (ddd, $J=17.5,4.5,2.5 \mathrm{~Hz}, 1 \mathrm{H}, \mathrm{H}_{12}$ ), 2.85 (ddd, $J=17.5,13.5,5.5 \mathrm{~Hz}, 1 \mathrm{H}$, , $\mathrm{H}_{12}$ ), 2.34 (dddd, $J=13.5,13.5,13.5,4.5 \mathrm{~Hz}, 1 \mathrm{H}, \mathrm{H}_{11}$ ), 2.16 (dddd, $J=13.5,4.5,2.5 \mathrm{~Hz}, 1 \mathrm{H}$, $\mathrm{H}_{11}$ ), 2.06 (td, $\left.J=7.5,2.6 \mathrm{~Hz}, 2 \mathrm{H}, \mathrm{H}_{2}\right), 2.00-1.87\left(\mathrm{~m}, 3 \mathrm{H}, \mathrm{H}_{1}, \mathrm{H}_{4}\right), 1.72\left(\mathrm{t}, J=7.4 \mathrm{~Hz}, 2 \mathrm{H}, \mathrm{H}_{3}\right)$. ${ }^{13} \mathrm{C}$ NMR (126 MHz, chloroform-d): $\delta 171.1(\mathrm{C}), 170.0(\mathrm{C}), 169.5(\mathrm{C}), 159.2(\mathrm{C}), 153.6(\mathrm{C})$, $133.5(\mathrm{C}), 130.8(\mathrm{CH}$ x2), $130.1(\mathrm{C}), 130.0(\mathrm{CH}), 129.2(\mathrm{C}), 116.7(\mathrm{CH}), 113.9(\mathrm{CH}$ x2, $\mathrm{CH})$, $82.8(\mathrm{C}), 69.5(\mathrm{CH}), 63.1\left(\mathrm{CH}_{2}\right), 55.4\left(\mathrm{CH}_{3}\right), 52.7(\mathrm{CH}), 45.2\left(\mathrm{CH}_{2}\right), 43.3\left(\mathrm{CH}_{2}\right), 32.9\left(\mathrm{CH}_{2}\right)$, $32.7\left(\mathrm{CH}_{2}\right), 32.4\left(\mathrm{CH}_{2}\right), 26.7(\mathrm{C}), 22.9\left(\mathrm{CH}_{2}\right), 13.4\left(\mathrm{CH}_{2}\right)$. IR (ATR-FTIR), $\mathrm{cm}^{-1}: 3287(\mathrm{br}), 2923$ $(\mathrm{m}) \mathrm{m}, 2853(\mathrm{~m}), 1677(\mathrm{~s}), 1606(\mathrm{~m}), 1513(\mathrm{~m}), 1275(\mathrm{~m}), 1246(\mathrm{~m}), 1161(\mathrm{~m}), 749(\mathrm{~m})$. HRMSESI $(\mathrm{m} / \mathrm{z}):[\mathrm{M}+\mathrm{H}]^{+}$calculated for $\mathrm{C}_{28} \mathrm{H}_{28} \mathrm{~N}_{4} \mathrm{O}_{5}, 501.2132$; found, 501.2077. 

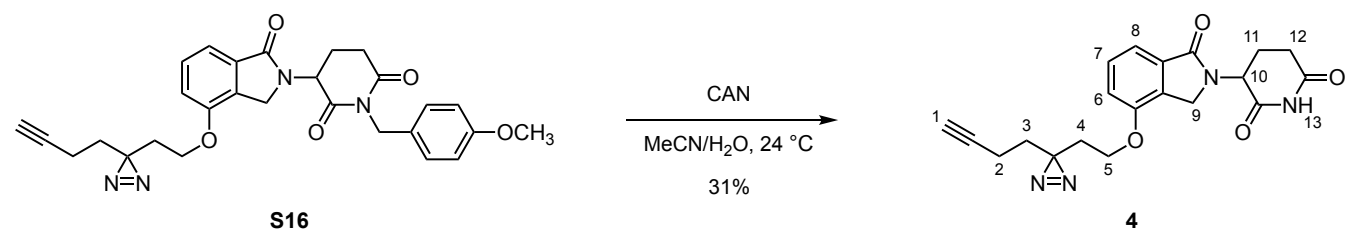

\section{Synthesis of analogue 4:}

Ammonium cerium(IV) nitrate $(65.7 \mathrm{mg}, 120 \mu \mathrm{mol}, 4.00$ equiv.) was added in a single portion to a solution of the compound $\mathbf{S} 16(15.0 \mathrm{mg}, 30.0 \mu \mathrm{mol}, 1.00$ equiv.) in acetonitrile (330 $\mu \mathrm{L})$ and water $(67.0 \mu \mathrm{L})$ at $25{ }^{\circ} \mathrm{C}$. The reaction mixture was stirred for $2 \mathrm{~h}$ at $25{ }^{\circ} \mathrm{C}$. The product mixture was diluted with ethyl acetate $(5.0 \mathrm{~mL})$ and transferred to a separatory funnel. The diluted product mixture was washed with water $(5.0 \mathrm{~mL})$ and saturated aqueous sodium chloride $(5.0 \mathrm{~mL})$. The organic layers were combined, and the combined organic layers were dried over sodium sulfate. The dried solution was filtered, and the filtrate was concentrated to dryness. The crude product was purified by flash-column chromatography (eluting with $1 \%$ methanol-dichloromethane). The product mixture was further purified by HPLC (grading 95\% to $5 \%$ water-acetonitrile over $25 \mathrm{~min}$, flow rate $=10 \mathrm{~mL} / \mathrm{min}$, retention time $=14.6 \mathrm{~min}$ ) to afford the titled compound as a colorless solid (4, $2.8 \mathrm{mg}, 31 \%)$.

$\mathrm{R}_{f}=0.25$ (2\% methanol-dichloromethane; UV). ${ }^{1} \mathrm{H}$ NMR (600 MHz, chloroform-d) $\delta 7.97$ (s, $\left.1 \mathrm{H}, \mathrm{H}_{13}\right), 7.51\left(\mathrm{~d}, J=7.8 \mathrm{~Hz}, 1 \mathrm{H}, \mathrm{H}_{6} / \mathrm{H}_{8}\right), 7.44\left(\mathrm{dd}, J=7.8,7.8 \mathrm{~Hz}, 1 \mathrm{H}, \mathrm{H}_{7}\right), 6.98(\mathrm{~d}, J=7.8 \mathrm{~Hz}$, $\left.1 \mathrm{H}, \mathrm{H}_{6} / \mathrm{H}_{8}\right), 5.23$ (dd, $\left.J=13.2,5.2 \mathrm{~Hz}, 1 \mathrm{H}, \mathrm{H}_{10}\right), 4.48\left(\mathrm{~d}, J=16.4 \mathrm{~Hz}, 1 \mathrm{H}, \mathrm{H}_{9}\right), 4.36(\mathrm{~d}, J=16.4$ $\mathrm{Hz}, 1 \mathrm{H}, \mathrm{H}_{9}$ ), 3.96 (ddd, $J=9.5,6.6,5.2 \mathrm{~Hz}, 1 \mathrm{H}, \mathrm{H}_{5}$ ), 3.92 (ddd, $J=9.5,7.2,4.8 \mathrm{~Hz}, 1 \mathrm{H}, \mathrm{H}_{5}$ ), $2.97-2.89\left(\mathrm{~m}, 1 \mathrm{H}, \mathrm{H}_{12}\right), 2.84$ (ddd, $\left.J=18.1,13.2,5.2 \mathrm{~Hz}, 1 \mathrm{H}, \mathrm{H}_{12}\right), 2.42$ (dddd, $J=13.2,13.2$, 13.2, 4.7 Hz, 1H, $\mathrm{H}_{11}$ ), 2.23 (dddd, $\left.J=13.2,5.2,5.2,2.5 \mathrm{~Hz}, 1 \mathrm{H}, \mathrm{H}_{11}\right), 2.07$ (td, $J=7.5,2.4$ $\mathrm{Hz}, 2 \mathrm{H}, \mathrm{H}_{2}$ ), 1.98 (t, $J=2.4 \mathrm{~Hz}, 1 \mathrm{H}, \mathrm{H}_{1}$ ), 1.97 (ddd, $J=15.6,7.2,5.2 \mathrm{~Hz}, 1 \mathrm{H}, \mathrm{H}_{4}$ ), 1.92 (ddd, $J$ $\left.=15.6,6.6,4.8 \mathrm{~Hz}, 1 \mathrm{H}, \mathrm{H}_{4}\right), 1.73\left(\mathrm{t}, \mathrm{J}=7.5 \mathrm{~Hz}, 2 \mathrm{H}, \mathrm{H}_{3}\right) \cdot{ }^{13} \mathrm{C}$ NMR $(126 \mathrm{MHz}$, chloroform-d) $\delta$ 171.0 (C), $169.4(\mathrm{C}), 169.3(\mathrm{C}), 153.4(\mathrm{C}), 133.2(\mathrm{C}), 129.9(\mathrm{C}), 129.9(\mathrm{CH}), 116.6(\mathrm{CH}), 113.9$ $(\mathrm{CH}), 82.7(\mathrm{C}), 69.4(\mathrm{CH}), 62.9\left(\mathrm{CH}_{2}\right), 51.9(\mathrm{CH}), 45.0\left(\mathrm{CH}_{2}\right), 32.7\left(\mathrm{CH}_{2}\right), 32.6\left(\mathrm{CH}_{2}\right), 31.6$ $\left(\mathrm{CH}_{2}\right), 26.6(\mathrm{C}), 23.4\left(\mathrm{CH}_{2}\right), 13.3\left(\mathrm{CH}_{2}\right)$. IR (ATR-FTIR), cm ${ }^{-1} 3243$ (br), 2925 (br), 1694 (s), $1606(\mathrm{~m}), 1493(\mathrm{~m}), 1277(\mathrm{~m}), 1145(\mathrm{~m}), 750(\mathrm{~m})$. HRMS-ESI $(\mathrm{m} / \mathrm{z}):[\mathrm{M}+\mathrm{H}]^{+}$calculated for $\mathrm{C}_{20} \mathrm{H}_{21} \mathrm{~N}_{4} \mathrm{O}_{4}, 381.1557$; found, 381.1566. 

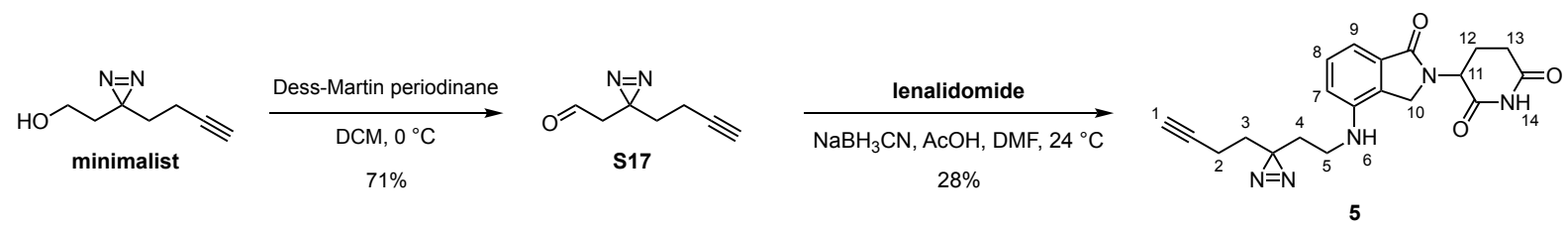

\section{Synthesis of analogue 5:}

\section{Step 1}

Sodium bicarbonate (164.2 mg, $1.95 \mathrm{mmol}, 15.0$ equiv.) was added in a single portion to a stirred solution of minimalist ${ }^{2}(18.0 \mathrm{mg}, 130 \mu \mathrm{mol}, 1.00$ equiv.) in dichloromethane $(1.3 \mathrm{~mL})$. The reaction mixture was cooled down to $0{ }^{\circ} \mathrm{C}$ and Dess-Martin periodinane $(82.9 \mathrm{mg}, 195$ $\mu \mathrm{mol}, 1.5$ equiv.) was added to the solution in a single portion. The reaction mixture was stirred for $2 \mathrm{~h}$ at $0{ }^{\circ} \mathrm{C}$. Additional Dess-Martin periodinane $(26.7 \mathrm{mg}, 65 \mu \mathrm{mol}, 0.5$ equiv.) was added to the reaction mixture in a single portion at $0{ }^{\circ} \mathrm{C}$ and the reaction mixture was stirred for 30 min at $0{ }^{\circ} \mathrm{C}$. Saturated aqueous sodium thiosulfate $(5.0 \mathrm{~mL})$ was added to the product mixture at $0{ }^{\circ} \mathrm{C}$ and stirred for $30 \mathrm{~min}$ at $25^{\circ} \mathrm{C}$. The product mixture was transferred to a separatory funnel and washed with water $(5.0 \mathrm{~mL})$. The aqueous layer was extracted with diethyl ether $(3 \times 5.0 \mathrm{~mL})$. The organic layers were combined, and the combined organic layers were dried over sodium sulfate. The crude product was filtrated through silica gel (eluting with $50 \%$ diethyl ether-hexane) to remove residual reagents. The aldehyde $\mathbf{S 1 7}$ was afforded as colorless oil $(12.6 \mathrm{mg}, 71 \%)$. The product was used in the following step without further purification.

\section{Step 2}

Lenalidomide ( $24.0 \mathrm{mg}, 92.5 \mu \mathrm{mol}, 1.00$ equiv.) and acetic acid (6.4 $\mu \mathrm{L}, 111 \mu \mathrm{mol}, 1.20$ equiv.) were added in a single portion to a stirred solution of the aldehyde $\mathbf{S 1 7}(12.6 \mathrm{mg}, 92.5 \mu \mathrm{mol}$, 1.00 equiv.) in $\mathrm{N}, \mathrm{N}$-dimethylformamide $(310 \mu \mathrm{L})$ at $25^{\circ} \mathrm{C}$. The reaction mixture was stirred for $20 \mathrm{~min}$ at $25^{\circ} \mathrm{C}$. Sodium cyanoborohydride $(8.7 \mathrm{mg}, 139 \mu \mathrm{mol}, 1.50$ equiv.) was added in a single portion to the reaction mixture and the reaction mixture was stirred for an additional 24 $\mathrm{h}$ at $25^{\circ} \mathrm{C}$. The product mixture was diluted with ethyl acetate $(5.0 \mathrm{~mL})$ and transferred to a separatory funnel. The diluted product mixture was washed with saturated aqueous sodium bicarbonate $(5.0 \mathrm{~mL})$ and saturated aqueous sodium chloride $(5.0 \mathrm{~mL})$. The layers were mixed, and the aqueous layer was extracted by ethyl acetate $(3 \times 5 \mathrm{~mL})$. The organic layers were combined, and the combined organic layers were dried over sodium sulfate. The dried solution was filtered, and the filtrate was concentrated to dryness. The crude product was purified by flash-column chromatography (eluting with $2 \%$ methanol-dichloromethane, grading to $3 \%$ methanol-dichloromethane, two steps). The obtained product mixture was further purified by HPLC (grading $95 \%$ to $48 \%$ water-acetonitrile over $28 \mathrm{~min}$, flow rate $=10 \mathrm{~mL} / \mathrm{min}$, retention time $=25.2 \mathrm{~min})$, to afford the titled compound as a colorless solid $(\mathbf{5}, 9.8 \mathrm{mg}, \mathbf{2 8 \%})$.

$\mathrm{R}_{f}=0.30$ (4\% methanol-dichloromethane; UV, anis aldehyde). ${ }^{1} \mathrm{H}$ NMR (500 MHz, methanol$\left.d_{4}\right): \delta 7.34\left(\mathrm{dd}, J=7.5,7.5 \mathrm{~Hz}, 1 \mathrm{H}, \mathrm{H}_{8}\right), 7.10\left(\mathrm{~d}, J=7.5 \mathrm{~Hz}, 1 \mathrm{H}, \mathrm{H}_{9}\right), 6.79(\mathrm{~d}, J=7.5 \mathrm{~Hz}, 1 \mathrm{H}$, $\left.\mathrm{H}_{7}\right), 5.16\left(\mathrm{dd}, J=13.5,5.0 \mathrm{~Hz}, 1 \mathrm{H}, \mathrm{H}_{11}\right), 4.33\left(\mathrm{~d}, J=17.0 \mathrm{~Hz}, 1 \mathrm{H}, \mathrm{H}_{10}\right), 4.28(\mathrm{~d}, J=17.0 \mathrm{~Hz}$, $1 \mathrm{H}, \mathrm{H}_{10}$ ), 3.08 (t, $J=7.0 \mathrm{~Hz}, 2 \mathrm{H}, \mathrm{H}_{5}$ ), 2.92 (ddd, $J=17.5,13.5,5.5 \mathrm{~Hz}, 1 \mathrm{H}, \mathrm{H}_{13}$ ), 2.79 (ddd, $J$ $\left.=17.5,4.5,2.5 \mathrm{~Hz}, 1 \mathrm{H}, \mathrm{H}_{13}\right), 2.48$ (dddd, $J=13.5,13.5,13.5,4.5 \mathrm{~Hz}, 1 \mathrm{H}, \mathrm{H}_{12}$ ), 2.29 (t, $J=2.5$ $\left.\mathrm{Hz}, 1 \mathrm{H}, \mathrm{H}_{1}\right), 2.23-2.13\left(\mathrm{~m}, 1 \mathrm{H}, \mathrm{H}_{12}\right), 2.03\left(\mathrm{td}, J=7.5,2.5 \mathrm{~Hz}, 2 \mathrm{H}, \mathrm{H}_{2}\right), 1.80(\mathrm{t}, J=7.0 \mathrm{~Hz}, 2 \mathrm{H}$, $\left.\mathrm{H}_{4}\right), 1.65\left(\mathrm{t}, J=7.5 \mathrm{~Hz}, 2 \mathrm{H}, \mathrm{H}_{3}\right) .{ }^{13} \mathrm{C}$ NMR (126 MHz, methanol-d $\left.d_{4}\right): \delta 174.7(\mathrm{C}), 172.4(\mathrm{C})$, $172.3(\mathrm{C}), 144.5(\mathrm{CH}), 133.1(\mathrm{C}), 130.7(\mathrm{CH}), 128.4(\mathrm{C}), 113.8(\mathrm{CH}), 112.4(\mathrm{CH}), 83.7(\mathrm{C})$, $70.4(\mathrm{CH}), 53.6(\mathrm{CH}), 47.3\left(\mathrm{CH}_{2}\right), 39.0\left(\mathrm{CH}_{2}\right), 33.6\left(\mathrm{CH}_{2}\right), 33.0\left(\mathrm{CH}_{2}\right), 32.4\left(\mathrm{CH}_{2}\right), 28.1(\mathrm{C})$, $24.3\left(\mathrm{CH}_{2}\right), 13.8\left(\mathrm{CH}_{2}\right)$. IR (ATR-FTIR), $\mathrm{cm}^{-1}: 3341$ (br), 3290 (br), 2920 (br), 2854 (br), 1672 (s), 1607 (s), $1349(\mathrm{~m}), 1201(\mathrm{~m}), 748(\mathrm{~m})$. HRMS-ESI $(\mathrm{m} / \mathrm{z})$ : [M+Na] $]^{+}$calculated for $\mathrm{C}_{20} \mathrm{H}_{21} \mathrm{~N}_{5} \mathrm{NaO}_{3}, 402.1536$; found, 402.1531. 

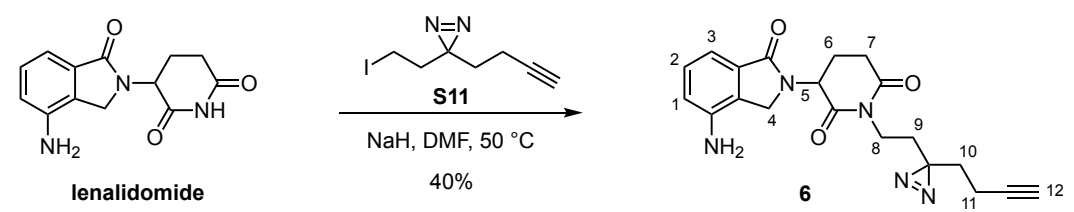

\section{Synthesis analogue 6:}

Sodium hydride ( $3.5 \mathrm{mg}, 146 \mu \mathrm{mol}, 1.50$ equiv.) was added in a single portion to a stirred solution of lenalidomide ( $25.2 \mathrm{mg}, 97.0 \mu \mathrm{mol}, 1.00$ equiv.) in $N, N$-dimethylformamide $(770 \mu \mathrm{L})$ at $0{ }^{\circ} \mathrm{C}$. The reaction mixture was stirred for $1 \mathrm{~h}$ at $25{ }^{\circ} \mathrm{C}$. A solution of minimalist iodide $\mathbf{S 1 1}$ (28.9 mg, $117 \mu \mathrm{mol}, 1.20$ equiv.) in $N, N$-dimethylformamide $(200 \mu \mathrm{L})$ was added to the reaction mixture and the reaction mixture was stirred for an additional $24 \mathrm{~h}$ at $50{ }^{\circ} \mathrm{C}$. The product mixture was cooled over $30 \mathrm{~min}$ to $25^{\circ} \mathrm{C}$. The cooled mixture was diluted with ethyl acetate $(5.0 \mathrm{~mL})$ and transferred to a separatory funnel. The mixture was washed with saturated aqueous sodium chloride $(5.0 \mathrm{~mL})$ and the aqueous layer was extracted by ethyl acetate $(3 \times 5.0 \mathrm{~mL})$. The organic layers were combined, and the combined organic layers were dried over sodium sulfate. The dried solution was filtered, and the filtrate was concentrated to dryness. The crude product was purified by flash-column chromatography (eluting with $50 \%$ ethyl acetate-hexanes, grading to $80 \%$ ethyl acetate-hexanes, two steps). The product was further purified with HPLC (eluting with $5 \%$ acetonitrile-water, grading to $95 \%$ acetonitrile-water over $25 \mathrm{~min}$, flow rate $=10 \mathrm{~mL} / \mathrm{min}$, retention time $=14.0 \mathrm{~min}$ ) afford the titled compound as a white solid (6, $14.8 \mathrm{mg}, 40 \%)$.

$\mathrm{R}_{f}=0.24$ (80\% ethyl acetate-hexane; UV, ninhydrin). ${ }^{1} \mathrm{H}$ NMR $\left(500 \mathrm{MHz}\right.$, methanol- $\left.d_{4}\right) \delta 7.26$ $\left(\mathrm{dd}, J=7.5,7.5 \mathrm{~Hz}, 1 \mathrm{H}, \mathrm{H}_{2}\right), 7.13\left(\mathrm{dd}, J=7.5,1.0 \mathrm{~Hz}, 1 \mathrm{H}, \mathrm{H}_{3}\right), 6.92(\mathrm{dd}, J=7.5,1.0 \mathrm{~Hz}, 1 \mathrm{H}$, $\left.\mathrm{H}_{1}\right), 5.16\left(\mathrm{dd}, J=13.5,5.0 \mathrm{~Hz}, 1 \mathrm{H}, \mathrm{H}_{5}\right), 4.34\left(\mathrm{~d}, J=16.5 \mathrm{~Hz}, 1 \mathrm{H}, \mathrm{H}_{4}\right), 4.27(\mathrm{~d}, J=16.5 \mathrm{~Hz}$, $\left.1 \mathrm{H}, \mathrm{H}_{4}\right), 3.86\left(\mathrm{dt}, J=13.5,7.5 \mathrm{~Hz}, 1 \mathrm{H}, \mathrm{H}_{8}\right), 3.81\left(\mathrm{dt}, J=13.5,7.5 \mathrm{~Hz}, 1 \mathrm{H}, \mathrm{H}_{8}\right), 3.02-2.88(\mathrm{~m}$, $2 \mathrm{H}, \mathrm{H}_{7}$ ), 2.48 (dddd, $J=13.0,13.0,13.0,5.5 \mathrm{~Hz}, 1 \mathrm{H}, \mathrm{H}_{6}$ ), 2.27 (t, $J=2.5 \mathrm{~Hz}, 1 \mathrm{H}, \mathrm{H}_{12}$ ), 2.18 (dddd, $J=13.0,13.0,5.0,3.0 \mathrm{~Hz}, 1 \mathrm{H}, \mathrm{H}_{6}$ ), 2.03 (dt, $J=7.5,2.5 \mathrm{~Hz}, 2 \mathrm{H}, \mathrm{H}_{11}$ ), 1.68 (dt, $J=15.0$, $7.5 \mathrm{~Hz}, 1 \mathrm{H}, \mathrm{H}_{10}$ ), 1.65 (dt, J = 15.0, 7.5 Hz, $1 \mathrm{H}, \mathrm{H}_{10}$ ), 1.60 (ddd, $J=14.5,7.5,7.5 \mathrm{~Hz}, 1 \mathrm{H}, \mathrm{H}_{9}$ ), 1.56 (ddd, $\left.J=14.5,7.5,7.5 \mathrm{~Hz}, 1 \mathrm{H}, \mathrm{H}_{9}\right) .{ }^{13} \mathrm{C}$ NMR $(125 \mathrm{MHz}$, chloroform-d): $\delta 171.1$ (C), 170.2 (C), $169.9(\mathrm{C}), 141.3(\mathrm{C}), 132.4(\mathrm{C}), 129.7(\mathrm{CH}), 126.4(\mathrm{C}), 118.3(\mathrm{CH}), 114.6(\mathrm{CH}), 82.8(\mathrm{C})$, $69.5(\mathrm{CH}), 52.7(\mathrm{CH}), 45.1\left(\mathrm{CH}_{2}\right), 36.1\left(\mathrm{CH}_{2}\right), 32.3\left(\mathrm{CH}_{2}\right), 31.6\left(\mathrm{CH}_{2}\right), 31.5\left(\mathrm{CH}_{2}\right), 27.2(\mathrm{C})$, $22.8\left(\mathrm{CH}_{2}\right), 13.4\left(\mathrm{CH}_{2}\right)$. IR (ATR-FTIR), cm ${ }^{-1}$ : 3352 (br), 3290 (br), 2921 (br), 1672 (s), 1491 $(\mathrm{m}), 1349(\mathrm{~m}), 1146(\mathrm{~m}), 750(\mathrm{~m})$. HRMS-ESI $(\mathrm{m} / \mathrm{z})$ : $[\mathrm{M}+\mathrm{Na}]^{+}$calculated for $\mathrm{C}_{20} \mathrm{H}_{21} \mathrm{~N}_{5} \mathrm{NaO}_{3}$, 402.1537; found, 402.1539 . 

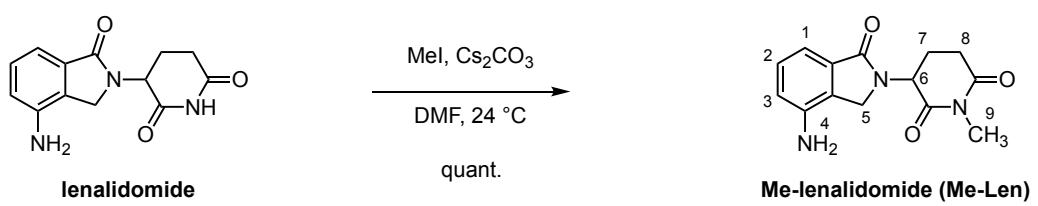

Me-lenalidomide (Me-Len)

\section{Synthesis of Me-Len:}

Methyl iodide (11.2 $\mu \mathrm{L}, 180 \mu \mathrm{mol}, 1.50$ equiv.) and cesium carbonate $(43.0 \mathrm{mg}, 132 \mu \mathrm{mol}, 1.10$ equiv) were added in the order in a single portion to a stirred solution of lenalidomide (31.1 $\mathrm{mg}, 120 \mu \mathrm{mol}, 1.00$ equiv.) in $\mathrm{N}, \mathrm{N}$-dimethylformamide $(1.2 \mathrm{~mL})$ at $24{ }^{\circ} \mathrm{C}$ and the reaction mixture was stirred for $2 \mathrm{~h}$ at $24{ }^{\circ} \mathrm{C}$. Additional methyl iodide $(3.7 \mu \mathrm{L}, 60 \mu \mathrm{mol}, 0.5$ equiv.) was added to the reaction mixture was stirred for $1 \mathrm{~h}$ at $24{ }^{\circ} \mathrm{C}$ The product mixture was diluted with ethyl acetate $(5.0 \mathrm{~mL}$ ) and transferred to a separatory funnel. The diluted product mixture was washed with saturated aqueous sodium chloride $(5.0 \mathrm{~mL})$. The aqueous layer was extracted by ethyl acetate $(6 \times 5 \mathrm{~mL})$. The combined organic layers were dried over sodium sulfate and concentrated to dryness. The crude product was purified by flash-column chromatography (eluting with 3\% methanol-chloroform, grading to $4 \%$ methanol-chloroform in one step) to afford the titled compound as a colorless solid (Me-lenalidomide, $33.1 \mathrm{mg}$, quant.).

$\mathrm{R}_{f}=0.20$ (5\% methanol-chloroform; UV, nihydrin). ${ }^{1} \mathrm{H}$ NMR $\left(600 \mathrm{MHz}\right.$, methanol- $\left.d_{4}\right) \delta 7.26$ (dd, $\left.J=7.7,7.7 \mathrm{~Hz}, 1 \mathrm{H}, \mathrm{H}_{2}\right), 7.13\left(\mathrm{dd}, J=7.7,0.9 \mathrm{~Hz}, 1 \mathrm{H}, \mathrm{H}_{1} / \mathrm{H}_{3}\right), 6.92(\mathrm{dd}, J=7.7,0.9 \mathrm{~Hz}$, $\left.1 \mathrm{H}, \mathrm{H}_{1} / \mathrm{H}_{3}\right), 5.17\left(\mathrm{dd}, J=13.4,5.1 \mathrm{~Hz}, 1 \mathrm{H}, \mathrm{H}_{6}\right), 4.32\left(\mathrm{~d}, J=16.6 \mathrm{~Hz}, 1 \mathrm{H}, \mathrm{H}_{5}\right), 4.26(\mathrm{~d}, J=16.6$ $\mathrm{Hz}, 1 \mathrm{H}, \mathrm{H}_{5}$ ), $3.15\left(\mathrm{~s}, 3 \mathrm{H}, \mathrm{H}_{9}\right), 3.01-2.88\left(\mathrm{~m}, 2 \mathrm{H}, \mathrm{H}_{8}\right), 2.46$ (dddd, $J=13.2,13.2,13.2,5.2 \mathrm{~Hz}$, $\left.1 \mathrm{H}, \mathrm{H}_{7}\right), 2.17$ (dddd, $\left.J=13.2,5.2,5.2,2.6 \mathrm{~Hz}, 1 \mathrm{H}, \mathrm{H}_{7}\right) .{ }^{13} \mathrm{C}$ NMR $(126 \mathrm{MHz}$, chloroform-d) $\delta$ $171.4(\mathrm{C}), 170.4(\mathrm{C}), 169.9(\mathrm{C}), 141.4(\mathrm{C}), 132.4(\mathrm{C}), 129.6(\mathrm{CH}), 126.4(\mathrm{C}), 118.2(\mathrm{CH}), 114.4$ $(\mathrm{CH}), 52.5(\mathrm{CH}), 45.14\left(\mathrm{CH}_{2}\right), 32.1\left(\mathrm{CH}_{2}\right), 27.2\left(\mathrm{CH}_{3}\right), 22.9\left(\mathrm{CH}_{2}\right)$. IR (ATR-FTIR), $\mathrm{cm}^{-1}$ : 3358, 1673, 1492, 1415, 1359, 1285, 1118. HRMS-ESI $(\mathrm{m} / \mathrm{z})$ : $[\mathrm{M}+\mathrm{H}]^{+}$calculated for $\mathrm{C}_{14} \mathrm{H}_{16} \mathrm{~N}_{3} \mathrm{O}_{3}$, 274.1186; found, 274.1186. 


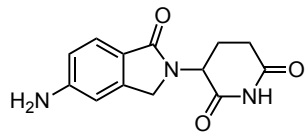

s1

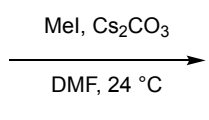

$61 \%$

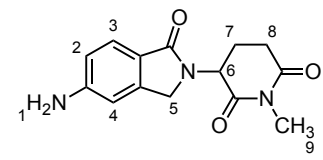

S18

\section{Synthesis of analogue S18:}

Methyl iodide ( $15.0 \mu \mathrm{L}, 241 \mu \mathrm{mol}, 1.50$ equiv.) and cesium carbonate $(57.5 \mathrm{mg}, 176 \mu \mathrm{mol}$, 1.10 equiv.) were added in the order in a single portion to a stirred solution of the compound S1 (41.6 mg, $160 \mu \mathrm{mol}, 1.00$ equiv.) in $\mathrm{N}, \mathrm{N}$-dimethylformamide $(1.6 \mathrm{~mL})$ at $24{ }^{\circ} \mathrm{C}$ and the reaction mixture was stirred for $2 \mathrm{~h}$ at $24{ }^{\circ} \mathrm{C}$. Additional methyl iodide $(15.0 \mu \mathrm{L}, 241 \mu \mathrm{mol}, 1.50$ equiv) was added to the reaction mixture was stirred for $6 \mathrm{~h}$ at $24{ }^{\circ} \mathrm{C}$ The product mixture was diluted with ethyl acetate $(5.0 \mathrm{~mL})$ and transferred to a separatory funnel. The diluted product mixture was washed with saturated aqueous sodium chloride $(5.0 \mathrm{~mL})$. The aqueous layer was extracted by ethyl acetate $(6 \times 5 \mathrm{~mL})$. The combined organic layers were dried over sodium sulfate and concentrated to dryness. The crude product was purified by flash-column chromatography (eluting with 3\% methanol-chloroform, grading to $4 \%$ methanol-chloroform in one step) to afford the titled compound as a colorless solid (S18, $26.8 \mathrm{mg}, 61 \%)$.

$\mathrm{R}_{f}=0.20$ (5\% methanol-chloroform; UV, nihydrin). ${ }^{1} \mathrm{H}$ NMR (400 MHz, DMSO-d6) $\delta 7.35$ (d, $\left.J=8.7 \mathrm{~Hz}, 1 \mathrm{H}, \mathrm{H}_{3}\right), 6.68-6.56\left(\mathrm{~m}, 2 \mathrm{H}, \mathrm{H} 2, \mathrm{H}_{4}\right), 5.82\left(\mathrm{~s}, 2 \mathrm{H}, \mathrm{H}_{1}\right), 5.07$ (dd, $J=13.4,5.2 \mathrm{~Hz}$, $\left.1 \mathrm{H}, \mathrm{H}_{6}\right), 4.24\left(\mathrm{~d}, J=16.6 \mathrm{~Hz}, 1 \mathrm{H}, \mathrm{H}_{5}\right), 4.10\left(\mathrm{~d}, J=16.6 \mathrm{~Hz}, 1 \mathrm{H}, \mathrm{H}_{5}\right), 3.01-2.92\left(\mathrm{~m}, 4 \mathrm{H}, \mathrm{H}_{9}, \mathrm{H}_{8}\right)$, 2.73 (ddd, $J=17.3,4.5,2.4 \mathrm{~Hz}, 1 \mathrm{H}, \mathrm{H}_{8}$ ), 2.32 (dddd, $J=13.2,13.2,13.2,4.5 \mathrm{~Hz}, 1 \mathrm{H}, \mathrm{H}_{7}$ ), 1.94 (dddd, $\left.J=13.2,5.2,5.2,2.4 \mathrm{~Hz}, 1 \mathrm{H}, \mathrm{H}_{7}\right) .{ }^{13} \mathrm{C}$ NMR (101 MHz, DMSO-d6) $\delta 172.0(\mathrm{C})$, $171.1(\mathrm{C}), 168.7(\mathrm{C}), 152.6(\mathrm{C}), 144.4(\mathrm{C}), 124.1(\mathrm{C}), 118.9(\mathrm{CH}), 113.6(\mathrm{CH}), 106.5(\mathrm{CH})$, $51.8(\mathrm{CH}), 46.6\left(\mathrm{CH}_{2}\right), 31.5\left(\mathrm{CH}_{2}\right), 26.6\left(\mathrm{CH}_{3}\right), 21.9\left(\mathrm{CH}_{2}\right)$. IR (ATR-FTIR), $\mathrm{cm}^{-1}: 3351,1667$, 1612, 1453, 1411, 1368, 1283, 1256, 1156, 1033, 771. HRMS-ESI $(\mathrm{m} / \mathrm{z}):[\mathrm{M}+\mathrm{H}]^{+}$calculated for $\mathrm{C}_{14} \mathrm{H}_{16} \mathrm{~N}_{3} \mathrm{O}_{3}, 274.1186$; found, 274.1187. 

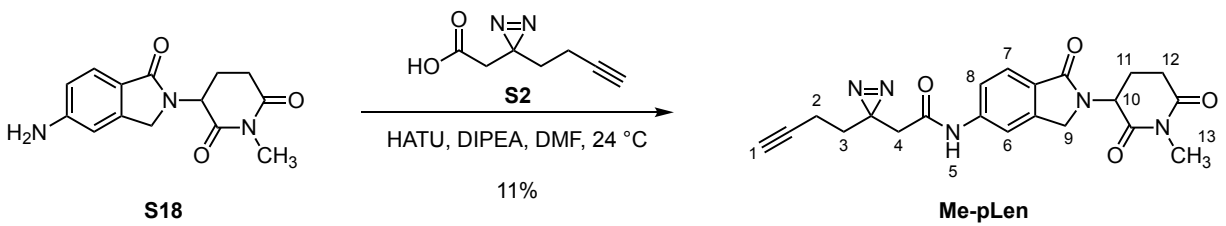

\section{Synthesis of Me-photolenalidomide (Me-pLen):}

The compound $\mathbf{S 1 8}(17.2 \mathrm{mg}, 63.1 \mu \mathrm{mol}, 1.00$ equiv.) was added in a single portion to a stirred solution of the carboxylic acid S2 (14.4 mg, $94.6 \mu \mathrm{mol}, 1.50$ equiv.) in $N, N$-dimethylformamide $(790 \mu \mathrm{L})$. HATU $(72.0 \mathrm{mg}, 189 \mu \mathrm{mol}, 3.00$ equiv.) and $N, N$-diisopropylethylamine $(33.0 \mu \mathrm{L}$, $189 \mu \mathrm{mol}, 3.00$ equiv) were added to the mixture in the order and the reaction mixture was stirred for $24 \mathrm{~h}$ at $24{ }^{\circ} \mathrm{C}$. The product mixture was diluted with ethyl acetate $(5.0 \mathrm{~mL})$ and transferred to a separatory funnel. The diluted product mixture was washed with saturated aqueous sodium chloride $(5.0 \mathrm{~mL})$ and $10 \%$ aqueous citric acid $(5.0 \mathrm{~mL})$. The layers were mixed and the aqueous layer was extracted by ethyl acetate $(3 \times 5 \mathrm{~mL})$. The combined organic layers were dried over sodium sulfate and concentrated to dryness. The crude product was purified by flash-column chromatography (eluting with $90 \%$ ethyl acetate-hexane). The obtained product mixture was further purified by HPLC (grading $95 \%$ to $5 \%$ water-acetonitrile over $30 \mathrm{~min}$, flow rate $=10 \mathrm{~mL} / \mathrm{min}$, retention time $=9.5 \mathrm{~min}$ ), to afford the titled compound as a colorless solid (Me-photolenalidomide, $2.7 \mathrm{mg}, 11 \%$ ).

$\mathrm{R}_{f}=0.60$ (100\% ethyl acetate; UV). ${ }^{1} \mathrm{H}$ NMR $\left(600 \mathrm{MHz}\right.$, acetone-d6) $\delta 9.51\left(\mathrm{~s}, 1 \mathrm{H}, \mathrm{H}_{5}\right), 8.07$ (d, $\left.J=1.8 \mathrm{~Hz}, 1 \mathrm{H}, \mathrm{H}_{8}\right), 7.67\left(\mathrm{~d}, J=8.2 \mathrm{~Hz}, 1 \mathrm{H}, \mathrm{H}_{7}\right), 7.56\left(\mathrm{dd}, J=8.2,1.8 \mathrm{~Hz}, 1 \mathrm{H}, \mathrm{H}_{6}\right), 5.17$ (dd, $\left.J=13.5,5.1 \mathrm{~Hz}, 1 \mathrm{H}, \mathrm{H}_{10}\right), 4.48\left(\mathrm{~d}, J=16.6 \mathrm{~Hz}, 1 \mathrm{H}, \mathrm{H}_{9}\right), 4.41\left(\mathrm{~d}, J=16.6 \mathrm{~Hz}, 1 \mathrm{H}, \mathrm{H}_{9}\right)$, 3.06 (s, 3H, $\mathrm{H}_{13}$ ), 3.02 (ddd, $J=17.4,13.7,5.4 \mathrm{~Hz}, 1 \mathrm{H}, \mathrm{H}_{12}$ ), 2.88 (ddd, $J=17.4,4.5,2.4 \mathrm{~Hz}$, $\left.1 \mathrm{H}, \mathrm{H}_{12}\right), 2.56\left(\mathrm{~s}, 2 \mathrm{H}, \mathrm{H}_{4}\right), 2.56-2.47\left(\mathrm{~m}, 1 \mathrm{H}, \mathrm{H}_{11}\right), 2.40\left(\mathrm{t}, J=2.7 \mathrm{~Hz}, 1 \mathrm{H}, \mathrm{H}_{1}\right), 2.17$ (dddd, $J$ $\left.=12.8,5.4,5.4,2.4 \mathrm{~Hz}, 1 \mathrm{H}, \mathrm{H}_{11}\right), 2.13\left(\mathrm{td}, J=7.6,2.7 \mathrm{~Hz}, 2 \mathrm{H}, \mathrm{H}_{2}\right), 1.82(\mathrm{z}, J=7.6 \mathrm{~Hz}, 2 \mathrm{H}$, $\left.\mathrm{H}_{3}\right) .{ }^{13} \mathrm{C}$ NMR (101 MHz, Acetone) $\delta 172.3(\mathrm{C}), 171.5(\mathrm{C}), 168.8(\mathrm{C}), 167.7(\mathrm{C}), 144.5(\mathrm{C})$, $143.1(\mathrm{C}), 128.3(\mathrm{C}), 124.7(\mathrm{CH}), 119.7(\mathrm{CH}), 114.4(\mathrm{CH}), 83.5(\mathrm{C}), 70.7(\mathrm{CH}), 53.3(\mathrm{CH}), 47.9$ $\left(\mathrm{CH}_{2}\right), 42.2\left(\mathrm{CH}_{2}\right), 33.2\left(\mathrm{CH}_{3}\right), 32.5\left(\mathrm{CH}_{2}\right), 27.2(\mathrm{C}), 27.0\left(\mathrm{CH}_{2}\right), 23.2\left(\mathrm{CH}_{2}\right), 13.6\left(\mathrm{CH}_{2}\right)$. IR (ATR-FTIR), $\mathrm{cm}^{-1}: 3286,1664,1550,1414,1358,1116,772,708$. HRMS-ESI $(\mathrm{m} / \mathrm{z}):[\mathrm{M}+\mathrm{H}]^{+}$ calculated for $\mathrm{C}_{21} \mathrm{H}_{22} \mathrm{~N}_{5} \mathrm{O}_{4}, 408.1666$; found, 408.1667 . 


\section{Antibodies and biological reagents.}

Antibodies included anti-IKZF1 (Cell Signaling Technology, Cat \# 9034S), anti-IKZF3 (Novus Bio, Cat \# NBP2-16938), anti-CRBN (Cell Signaling Technology, Cat \# 71810S, Sigma-Aldrich, Cat \# HPA045910), anti-Flag (Sigma-Aldrich, Cat \# F3165), anti- $\beta$-actin (Santa Cruz, Cat \# sc-47778), anti-GSPT1 (Proteintech, Cat \# 10763-1-AP), anti-CK1 $\alpha$ (Abcam, Cat \# ab206652), anti-elF3i (Proteintech, Cat \# 10086-380), anti-DDB1 (Bethyl Laboratories, Cat \# A300-462A), anti-CUL4A (Bethyl Laboratories, Cat \# A300-739A).

Antibody-conjugated beads for immunoprecipitation included Anti-Flag M2 magnetic beads (Sigma-Aldrich, Cat \# M8823), anti-EPEA CaptureSelect ${ }^{\mathrm{TM}} \mathrm{C}$-tag affinity matrix (Thermo Scientific, Cat \# 191307005), and Pierce Anti-HA magnetic beads (Pierce, Cat \# PI88836). Protein A/G magnetic beads (Bimake, Cat \# B23202) were used for endogenous protein immunoprecipitation. For AlphaScreen assay, nickel chelate acceptor beads (PerkinElmer, Cat \# AS101D) and glutathione donor beads (PerkinElmer, Cat \#6765300) were used. For flow cytometry staining, PE rat anti-human IL-2 antibody (BD Biosciences, 559334), FITC mouse anti-human CD69 antibody (BD Biosciences, 555530, Clone FN50) and FITC rat antimouse TNF- $\alpha$ antibody (eBioscience ${ }^{\mathrm{TM}}$, MP6-XT22) were used. Cell lysis buffer was prepared by diluting from 10X cell lysis buffer (Cell Signaling Technology, Cat \# 9803S). TBS buffer was prepared by diluting from 10X TBS buffer solution (Quality Biological, Cat \# 10128-546). To quantify DNA-binding affinity, TransAM DNA-binding ELISA kit was purchased (Active Motif, Cat \# 40096). For qRT-PCR, RNeasy Plus Mini kit (Qiagen, Cat \# 74134), PrimeScript RT reagent kit (TakaraBio Cat \# RR037A), and QuantiTect SYBR Green PCR kit (Qiagen, Cat \# 204143) were used. Sequencing-grade modified trypsin (Promega, Cat \# V5111), sequencinggrade chymotrypsin (Promega, Cat \# V1061) and mini Bio-Spin columns (Bio-Rad, Cat \# 7326207) were purchased.

\section{Cell culture and transfection.}

HEK293T cells (a gift from the Bertozzi lab, Stanford University), HEK293T cells stably expressing Flag-CRBN (HEK293T-CRBN) and HEK293T cells with CRBN knock-down by lentiviral shRNA (a gift from the Deshaies lab, California Institute of Technology), HEK293T cells stably transfected with NLucP/NF-kB vector (HEK293T-NF-kB, a gift from the Choudhary lab, Broad Institute), and RAW264.7 cells (a gift from the Mitchison lab, Harvard Medical School) were cultured in Dulbecco's Modified Eagle Medium (DMEM, Cat \# 11995073), supplemented with penicillin $(50 \mu \mathrm{g} / \mathrm{mL})$, streptomycin $(50 \mu \mathrm{g} / \mathrm{mL})$, and $10 \%(\mathrm{v} / \mathrm{v}) \mathrm{FBS}$. HEK293T-CRBN cell line was established by transducing cells with the corresponding lentiviral particles with transduction efficiency monitored by GFP signals via flow cytometry. HEK293T-NF-KB were maintained under the selection of $1 \mu \mathrm{g} / \mu \mathrm{L}$ hygromycin. MM.1S cells (ATCC), U937 cells (ATCC), Jurkat cells (ATCC) and MOLM13 cells (a gift from the Shair lab, Harvard University) were cultured in RPMI1640 (Cat \#45000-396) supplemented with penicillin $(50 \mu \mathrm{g} / \mathrm{mL})$, streptomycin $(50 \mu \mathrm{g} / \mathrm{mL})$ and $10 \%(\mathrm{v} / \mathrm{v})$ FBS.

Transfection was performed using TransIT-PRO (Mirus Bio, Cat \# MIR 5740) according to the manufacturer's instructions. Cells were lysed with $1 \mathrm{X}$ cell lysis buffer diluted from 10X cell lysis buffer supplemented with 1X cOmplete protease inhibitor cocktail (PI, Cat \# 11697498001) unless otherwise noted.

\section{Plasmids and subcloning.}

Human CRBN with a Flag tag, human elF3i with a His tag, and HA-tagged ubiquitin were obtained as detailed in the table below. IKZF1 with an EPEA tag was subcloned into the pcDNA3.1 vector. Empty pcDNA3.1 vector was used as a negative control for transfection.

Genetic constructs and primers used in this study are listed below: 


\begin{tabular}{|c|c|c|c|c|}
\hline No. & Plasmid name & $\begin{array}{l}\text { Abbreviated } \\
\text { name }\end{array}$ & Details & Source \\
\hline 1 & pcDNA3.1 & Ctrl & $\begin{array}{l}\text { Empty vector for mammalian } \\
\text { expression }\end{array}$ & Invitrogen, Cat \# V79020 \\
\hline 2 & $\begin{array}{l}\text { pCDH-Flag-CRBN-WT-T2AcGFP- } \\
\text { MSCV }\end{array}$ & Flag-CRBN & Full length CRBN with a Flag tag & A gift from Deshaies lab \\
\hline 3 & pcDNA3.1-IKZF1-EPEA & IKZF1-EPEA & & Made in house \\
\hline 4 & pNL3.2.NF-kB-RE[NlucP/NF-kB-RE & Hygro] Vector & NanoLuc NF-kB reporter gene & Promega, Cat \# N111A \\
\hline 5 & pCMV3-His-elF3i & His-elF3i & $\mathrm{N}-\mathrm{His}$ tagged human elF3i & $\begin{array}{l}\text { Sino Biological, Cat \# HG16651- } \\
\mathrm{NH}\end{array}$ \\
\hline 6 & pcDNA3.1-HA-Ubiquitin & & & Addgene, Cat \# 18712 \\
\hline 7 & pET28a-His-CULT & & $\begin{array}{l}\text { His tagged human CULT for } \\
\text { bacterial expression }\end{array}$ & Made in house \\
\hline
\end{tabular}

\begin{tabular}{|c|c|c|c|}
\hline No. & Primer name & Details & Sequence \\
\hline 1 & ZL_hRT001F & qRT-PCR primer for human IRF4 & GAACGAGGAGAAGAGCATCTTCC \\
\hline 2 & ZL_hRT001R & qRT-PCR primer for human IRF4 & CGATGCCTTCTCGGAACTTTCC \\
\hline 3 & ZL_hRT002F & qRT-PCR primer for human NF-KB & GCAGCACTACTTCTTGACCACC \\
\hline 4 & ZL_hRT002R & qRT-PCR primer for human NF-KB & TCTGCTCCTGAGCATTGACGTC \\
\hline 5 & ZL_hRT003F & qRT-PCR primer for human TNF- $\alpha$ & СTCTTCTGCCTGCTGCACTTTG \\
\hline 6 & ZL_hRT003R & qRT-PCR primer for human TNF- $\alpha$ & ATGGGCTACAGGCTTGTCACTC \\
\hline 7 & ZL_hRT022F & qRT-PCR primer for human elF3i & CTGTGGCCAAGGACCCTATC \\
\hline 8 & ZL_hRT022R & qRT-PCR primer for human elF3i & CCAGTGAGGACATGCTTGGT \\
\hline 9 & ZL_hRT025F & qRT-PCR primer for human GAPDH & GTCTCCTCTGACTTCAACAGCG \\
\hline 10 & ZL_hRT025R & qRT-PCR primer for human GAPDH & ACCACCCTGTTGCTGTAGCCAA \\
\hline 11 & ZL_mRT004F & qRT-PCR primer for mouse NF-kB & GCTGCCAAAGAAGGACACGACA \\
\hline 12 & ZL_mRT004R & qRT-PCR primer for mouse NF-KB & GGCAGGCTATTGCTCATCACAG \\
\hline 13 & ZL_mRT005F & qRT-PCR primer for mouse TNF- $\alpha$ & GGTGCCTATGTCTCAGCCTCTT \\
\hline 14 & ZL_mRT005R & qRT-PCR primer for mouse TNF- $\alpha$ & GCCATAGAACTGATGAGAGGGAG \\
\hline 15 & ZL_mRT006F & qRT-PCR primer for mouse GAPDH & CATCACTGCCACCCAGAAGACTG \\
\hline 16 & ZL_mRT006R & qRT-PCR primer for mouse GAPDH & ATGCCAGTGAGCTTCCCGTTCAG \\
\hline 17 & ZL_mRT007F & qRT-PCR primer for mouse IRF4 & GAACGAGGAGAAGAGCGTCTTC \\
\hline 18 & ZL_mRT007R & qRT-PCR primer for mouse IRF4 & GTAGGAGGATCTGGCTTGTCGA \\
\hline 19 & CULT-F & pET28a-CULT cloning & GGAATTCCATATGATGAACAAGTGTACGAGCCTGT \\
\hline 20 & CULT-R & pET28a-CULT cloning & GAGCTCGAATTCTTACAGGCAC \\
\hline
\end{tabular}

\section{Overexpression and purification of CULT.}

pET28a-CULT was constructed by extracting the region encoding the CULT domain from a E. coli codon-optimized gene encoding human cereblon (synthesized by Atum Bio) using primers CULT-F and CULT-R. The fragment was then introduced into pET28a using restriction enzyme cloning with Ndel and EcoRI. BL21 (DE3) cells transformed with pET28a-CULT were used to inoculate overnight cultures of LB with $50 \mu \mathrm{g} / \mathrm{mL}$ kanamycin, which were incubated at $30{ }^{\circ} \mathrm{C}$ with shaking at $200 \mathrm{rpm}$ for approximately $16 \mathrm{~h}$. For each large-scale overexpression, kanamycin was added to $750 \mathrm{~mL}$ autoclaved LB to a final concentration of $50 \mu \mathrm{g} / \mathrm{mL}$ and the culture was inoculated with overnight culture diluted 1:100. The overexpression cultures were incubated at $30^{\circ} \mathrm{C}$ with shaking at $200 \mathrm{rpm}$ until the OD600 was approximately 0.4 , at which point isopropyl isopropyl $\beta$-D-1-thiogalactoside (IPTG) was added to a final concentration of $0.1 \mathrm{mM}$ and the temperature was reduced to $25^{\circ} \mathrm{C}$. The cultures were incubated for $4-6 \mathrm{~h}$ 
prior to collecting the cells by centrifugation, flash freezing with liquid nitrogen, and storing at $-80^{\circ} \mathrm{C}$.

Up to 6 pellets, each from a $750 \mathrm{~mL}$ overexpression culture, were purified simultaneously. To purify protein, cell pellets were thawed on ice or in cool water, then resuspended in $5 \mathrm{~mL}$ of $25 \mathrm{mM}$ imidazole, $1 \times$ protease inhibitor, 1\% Triton-X 100/PBS per pellet. Up to 3 pellets were combined and sonicated ( $30 \mathrm{~s}$ on, $10 \mathrm{~s}$ off, $5 \mathrm{~min}$ total, $25 \%$ amplitude) on ice together. Lysates were clarified by centrifugation $\left(15,000 \times \mathrm{g}, 4^{\circ} \mathrm{C}, 10 \mathrm{~min}\right)$ and syringe filtration $(0.45 \mu \mathrm{m})$. The His-tagged protein was crudely purified on a Ni-bound $1 \mathrm{~mL}$ HiTrap Chelating HP column according to the manufacturer's instructions. The column was equilibrated and washed with $25 \mathrm{mM}$ imidazole/PBS before the proteins were eluted with a gradient to $500 \mathrm{mM}$ imidazole/PBS. Protein-containing fractions, as determined by A280, were taken directly to further purification on a HiLoad 16/600 Superdex 200 pg column, pre-equilibrated and run with PBS. Protein-containing fractions not eluting with the dead volume were collected and concentrated to approximately $100 \mu \mathrm{M}$ with a $10 \mathrm{kDa}$ MWCO spin concentrator, with protein concentration was determined by measuring the $A 280\left(\varepsilon=27,960 \mathrm{M}^{-1} \mathrm{~cm}^{-1}\right.$ with reduced cysteines). The protein was aliquoted, flash frozen with liquid nitrogen, and stored at $-80^{\circ} \mathrm{C}$.

\section{Structural proteomics mass spectrometry sample preparation.}

For each analysis, $20 \mu \mathrm{M}$ CULT and $22 \mu \mathrm{M}$ of the indicated compound in $0.1 \%$ Triton-X 100 in PBS was prepared ( $34 \mu \mathrm{L}, 10 \mu \mathrm{g}$ of CULT) with the compounds added from $4 \mathrm{mM}$ stock in DMSO. The sample was incubated for $30 \mathrm{~min}$ at $25^{\circ} \mathrm{C}$, followed by photoirradiation for $30 \mathrm{~s}$ at $4{ }^{\circ} \mathrm{C}$ via Dymax. A stock solution of $10 \%$ RapiGest/PBS was added to the photoirradiated sample to a final concentration of $1 \%(3.8 \mu \mathrm{L})$. The denatured proteins were tagged with an isotopically coded, cleavable biotin picolyl-azide probe ${ }^{4}$ via CuAAC by addition of a pre-mixed cocktail (final concentrations: $100 \mu \mathrm{M}$ cleavable biotin picolyl-azide probe, $250 \mu \mathrm{M}$ THPTA, $250 \mu \mathrm{M} \mathrm{CuSO}_{4}, 2.5 \mathrm{mM}$ freshly dissolved sodium ascorbate) and incubated for $1.5 \mathrm{~h}$ at $25^{\circ} \mathrm{C}$ with inversion. Protein was precipitated by the addition of cold acetone $(160 \mu \mathrm{L})$ and incubated for $30 \mathrm{~min}$ at $-80^{\circ} \mathrm{C}$. The precipitated protein was pelleted by centrifugation $\left(4{ }^{\circ} \mathrm{C}, 10 \mathrm{~min}\right.$, $21,300 \mathrm{xg}$ ). The supernatant was discarded, and the protein pellet was air dried for $10 \mathrm{~min}$ at $25^{\circ} \mathrm{C}$. The protein was resuspended in $100 \mathrm{mM}$ TEAB $(50 \mu \mathrm{L}, \mathrm{pH}=8.5)$ and $100 \mathrm{mM}$ DTT/PBS $(1.5 \mu \mathrm{L}$, final concentration $3 \mathrm{mM})$ was added before incubating for 30 min at $25{ }^{\circ} \mathrm{C}$ with inversion. $500 \mathrm{mM}$ iodoacetamide/PBS $(1.0 \mu \mathrm{L}$, final concentration $10 \mathrm{mM})$ was then added to the sample and incubated for $30 \mathrm{~min}$ at $25^{\circ} \mathrm{C}$ in the dark with inversion. The protein was then digested by adding sequence-grade chymotrypsin $(0.5 \mu \mathrm{g})$ in $1 \mathrm{mM} \mathrm{HCl}$ and incubating for $15-18 \mathrm{~h}$ at $25^{\circ} \mathrm{C}$ with inversion. The digested protein was acidified by formic acid $(1.0 \mu \mathrm{L})$ and incubated for $30 \mathrm{~min}$ at $25^{\circ} \mathrm{C}$ with inversion to cleave the cleavable biotin picolyl-azide probe. The sample was desalted using a C18 Ziptip according to the manufacture's protocol with elution in $0.1 \%$ formic acid $/ 70 \%$ acetonitrile/water and the desalted peptides were dried on a Speed-Vac. The dried sample was re-suspended in $20 \mu \mathrm{L} 0.1 \%$ formic acid/water for analysis.

\section{Structural proteomics mass spectrometry procedures.}

The sample was loaded onto a microcapillary trapping column (C18 Reprosil resin, Dr. Maisch, $5 \mu \mathrm{m}$ particle size, $100 \AA$ pore size, $30 \mathrm{~mm}$ length, $100 \mu \mathrm{m}$ internal diameter) and then separated on an analytical column (50 cm $\mu$ PACTM column, PharmaFluidics) at $200 \mathrm{~nL} / \mathrm{min}$. The column temperature was maintained at $40^{\circ} \mathrm{C}$. Peptides were eluted with a water/acetonitrile gradient (buffer $A=0.1 \%$ formic acid/water, buffer $B=0.1 \%$ formic acid/acetonitrile; flow rate $200 \mathrm{~nL} / \mathrm{min}$; gradient: hold at $2 \% \mathrm{~B}$ for $5 \mathrm{~min}$, increase to $6 \% \mathrm{~B}$ over $1 \mathrm{~min}$, increase to $35 \%$ B over $29 \mathrm{~min}$, increase to $95 \%$ B over $5 \mathrm{~min}$, hold at $95 \%$ B for 20 $\mathrm{min}$ ). Electrospray ionization was enabled through applying a voltage of $1.8 \mathrm{kV}$ using a homemade electrode junction at the end of the microcapillary column and sprayed from stainlesssteel needle (PepSep, Denmark). The Lumos Orbitrap was operated in data-dependent mode 
and survey scans of peptide precursors were performed at $120 \mathrm{~K}$ FWHM resolution over a $\mathrm{m} / \mathrm{z}$ range of 400-1800, an AGC setting of 1,000,000, and the maximum ion accumulation time of $50 \mathrm{~ms}$. Tandem MS was performed on the 10 most abundant precursors with an AGC setting of 10,000 and fragmentation energy of $35 \%$ for CID, at $15 \mathrm{~K}$ FWHM resolution, an AGC setting of 10,000 and fragmentation energy of $37 \%$ for HCD. An isolation window of $3 \mathrm{~m} / \mathrm{z}$ used to ensure capture of isotopically coded species. With a mass tolerance of $10 \mathrm{ppm}$, selected precursors were excluded from further fragmentation for $60 \mathrm{~s}$.

\section{Structural proteomics data analysis procedures.}

The raw data were analyzed using Proteome Discoverer 2.4.1.15. Assignment of MS/MS spectra was performed using the Sequest HT algorithm by searching the data against a protein sequence database of CULT and common contaminant proteins. Search parameters included: mass tolerance of $10 \mathrm{ppm}$ for the precursor, 0.02 Da for HCD fragment ions, 0.6 Da for CID fragment ions, semi-specific digestion, 2 missed cleavages, static modification of carbamidomethylation on cysteine residues $(+57.0214 \mathrm{Da})$, a dynamic oxidation on methionine residues (+15.9949 Da), a dynamic deamidation on asparagine and glutamine residues $(+0.9847 \mathrm{Da})$ and a dynamic modification of the compounds on any amino acid residues (photolenalidomide: $+588.2355 \mathrm{Da}$, analogue 1: +602.2512 Da). Peptide spectral matches (PSMs) were validated with the Target Decoy PSM Validator. Spectra assigned as photolenalidomide/analogue 1-conjugated peptides were manually validated by evaluating the isotopic coding embedded in the MS1 precursor.

\section{Structural modeling details.}

Docking study was performed with MOE version 2020.09. A ligand molecule in the indicated crystal structure was adapted to the indicated compounds and the adapted complex was subjected to energy minimization with Amber10:EHT force field followed by preparation with Protonate 3D. Ligand conformations were generated with the bond rotation method and the docking was performed with the Triangle Matcher placement method and scored with the London dG scoring function. The lowest energy conformations were rendered and 30 of them were submitted to a refinement step with the Induced Fit method and rescored with the GBVI/WSA dG scoring function. A conformation of each complex with the lowest S score given by the GBVI/WSA dG scoring function was analyzed for the ligand interactions.

\section{Immunoprecipitation assays in live cells.}

For immunoprecipitation of proteins with the Flag tag, cell lysates with equal amounts of protein were diluted with the lysis buffer and incubated with Anti-Flag M2 magnetic beads (prewashed three times with TBS) for $2 \mathrm{~h}$ at $4{ }^{\circ} \mathrm{C}$ with rotation. The beads were washed three times with TBS buffer (50 mM Tris $\mathrm{HCl} \mathrm{pH} \mathrm{7.4,} 150 \mathrm{mM} \mathrm{NaCl}$ ). The enriched proteins were eluted with SDS sample buffer for SDS-PAGE or $50 \mu \mathrm{L} 3 \mathrm{X}$ Flag peptide solution (final concentration $150 \mathrm{ng} / \mu \mathrm{l}$ ), according to the manufacturer's instructions.

For immunoprecipitation of proteins with the EPEA tag, cell lysates with equal amounts of protein were diluted with PBS and incubated with C-tag affinity matrix (pre-washed three times with PBS) for $2 \mathrm{~h}$ at $25^{\circ} \mathrm{C}$. The beads were washed three times with PBS buffer. The enriched proteins were eluted with SDS sample buffer for SDS-PAGE or $50 \mu \mathrm{L}$ neutral $\mathrm{pH}$-based elution buffer (20 mM Tris, $2 \mathrm{M} \mathrm{MgCl}_{2}, \mathrm{pH} 7.0$ ), according to the manufacturer's instructions.

For endogenous protein immunoprecipitation using protein $A / G$ beads, cell lysates with equal amounts of protein were diluted with PBS and incubated with protein A/G beads (pre-washed three times with binding buffer, $50 \mathrm{mM}$ Tris, $150 \mathrm{mM} \mathrm{NaCl}, 0.2 \%$ Triton, $\mathrm{pH}=7.5$ ) for $2 \mathrm{~h}$ at 4 ${ }^{\circ} \mathrm{C}$ along with the protein-specific antibody at the vendor-suggested dilution. The beads were washed three times with binding buffer (50 mM Tris, $150 \mathrm{mM} \mathrm{NaCl}, 0.2 \%$ Triton, $\mathrm{pH}=7.5)$. The 
enriched proteins were eluted with acidic elution buffer (100 mM glycine, $0.1 \%$ Triton, $\mathrm{pH}=2.8$ ) before neutralizing with $1 \mathrm{M}$ Tris $(\mathrm{pH}=8)$, according to the manufacturer's instructions.

\section{AlphaScreen assay for binding interaction analysis}

AlphaScreen buffer was freshly made containing $50 \mathrm{mM}$ HEPES $200 \mathrm{mM} \mathrm{NaCl}, 0.1 \% \mathrm{w} / \mathrm{v} \mathrm{BSA}$, $1 \mathrm{mM}$ TCEP $(\mathrm{pH}=7.4)$ right before the assay. Compounds at the concentrations indicated were mixed in AlphaScreen buffer along with indicated proteins at their respective concentrations (His-CRBN/DDB1 $50 \mathrm{nM}$, GST-IKZF1 $100 \mathrm{nM}$, GST-elF3i $100 \mathrm{nM}$ ), and $10 \mu \mathrm{L}$ of the reaction mixture was incubated at $25{ }^{\circ} \mathrm{C}$ for $1 \mathrm{~h}$ in a 384-well white opaque OptiPlate384 plate. Following the incubation, $5 \mu \mathrm{L}$ of the detection mixture containing nickel chelate acceptor beads (PerkinElmer, Cat \# AS101D) and glutathione donor beads (PerkinElmer, Cat \#6765300) were added to each well in dark. After incubation at $25{ }^{\circ} \mathrm{C}$ for $1 \mathrm{~h}$ in dark, luminescent signals were detected using a multimode microplate reader SpectraMax i3x.

\section{Cycloheximide (CHX) treatment.}

HEK293T cells were treated with DMSO or indicated drug $1 \mathrm{~h}$ before the addition of $50 \mu \mathrm{g} / \mathrm{mL}$ $\mathrm{CHX}$ for up to $24 \mathrm{~h}$. At the indicated time points, cells were harvested and washed with PBS twice before lysis with $1 \mathrm{X}$ cell lysis buffer with $1 \mathrm{X}$ PI. The samples were then subjected to Western blot analysis.

\section{Photoaffinity labeling experiment and in-gel fluorescent imaging.}

Cells were plated on a 6-well plate and treated with the indicated drugs for $1 \mathrm{~h}$ at $37^{\circ} \mathrm{C}$ before photoirradiation for $120 \mathrm{~s}$ at $4{ }^{\circ} \mathrm{C}$ via Dymax. After immunoprecipitation, the eluents were then tagged with AlexaFluor ${ }^{\circledR} 488$ azide via CuAAC by addition of a pre-mixed cocktail (final concentrations: $25 \mu \mathrm{M}$ AlexaFluor ${ }^{\circledR} 488$ azide, $100 \mu \mathrm{M}$ THPTA, 1 mM CuSO 4 , 2 mM freshly dissolved sodium ascorbate) and incubated for $1 \mathrm{~h}$ at $25^{\circ} \mathrm{C}$ with inversion. The reactions were quenched by the addition of pre-chilled acetone and incubated for $20 \mathrm{~min}$ at $-80{ }^{\circ} \mathrm{C}$. The protein precipitates were isolated by centrifugation $\left(10 \mathrm{~min}, 4{ }^{\circ} \mathrm{C}, 21,300 \times \mathrm{g}\right)$ and the supernatant was discarded. The protein pellet was dried for $10 \mathrm{~min}$ at $25{ }^{\circ} \mathrm{C}$ and then resuspended in $50 \mu \mathrm{L} 1 \%$ RapiGest/PBS by sonication ( $2 \mathrm{~s}$ on, $5 \mathrm{~s}$ off, $10 \%$ amplitude, $10 \mathrm{~s}$ total) before visualization by SDS-PAGE.

\section{Cell viability assay (MTT).}

Cells were treated with the indicated compound in triplicate on a 96-well plate at $1 \times 10^{6}$ cells $/ \mathrm{mL}$ in $100 \mu \mathrm{L}$ per well for 5 days at $37^{\circ} \mathrm{C}$. 3-(4,5-Dimethylthiazol-2-yl)-2,5diphenyltetrazolium bromide (MTT) solution (10 $\mu \mathrm{L}$, freshly dissolved in PBS at $4 \mathrm{mg} / \mathrm{mL}$ ) was added to each well and the cells were incubated for $4 \mathrm{~h}$ at $37^{\circ} \mathrm{C}$. After incubation, MTT solubilization solution ( $100 \mu \mathrm{L}, 10 \%$ SDS with $0.04 \mathrm{~N} \mathrm{HCl})$ was added to each well and incubated for $16 \mathrm{~h}$ at $37^{\circ} \mathrm{C}$. The absorbance was measured at a wavelength of $570 \mathrm{~nm}$ on a multimode microplate reader FilterMax. Background absorbance signal from media-only blank wells was subtracted and the absorbance signal was normalized to that of untreated cells.

\section{Flow cytometry (FACS).}

Cells were fixed with $4 \%$ formaldehyde for $10 \mathrm{~min}$ at $37^{\circ} \mathrm{C}$, and permeabilized by $90 \% \mathrm{MeOH}$ at $4{ }^{\circ} \mathrm{C}$ for $30 \mathrm{~min}$. The samples were then washed three times with PBS, blocked in $1 \%$ BSA for $15 \mathrm{~min}$ at $25^{\circ} \mathrm{C}$, and incubated with FITC- or PE-conjugated antibodies in $1 \%$ BSA for 30 min at $4{ }^{\circ} \mathrm{C}$ covered with foil. The samples were washed three times with PBS and analyzed. Cells were gated with SSC-A/FSC-A for live population and FSC-H/FSC-A for singlets, and 100,000 cells were analyzed from each sample. Relative number of FITC- or PE-expressing 
cells for different treatment conditions was calculated by BD FACSDiva Software and analyzed by Flowjo.

\section{RNA isolation and qRT-PCR experiment.}

Cells were treated as indicated before RNA isolation. Qiagen RNeasy-Plus Mini kits were used to purify RNA from cells, according to the instructions from the manufacturer. Isolated RNA then underwent two-step real-time RT-PCR by using PrimeScript RT reagent kit for reverse transcription and QuantiTect SYBR Green PCR kit.

\section{Luciferase reporter assay.}

HEK293T cells transfected with NLucP/NF-KB vector were incubated for $6 \mathrm{~h}$ in medium without serum prior to use. The cells were treated with either DMSO or the indicated compound and induced with $10 \mu \mathrm{M}$ TNF- $\alpha$ for $5 \mathrm{~h}$ at $37^{\circ} \mathrm{C}$ in the incubator before luciferase activity detection. Luciferase reporter assays were performed using the Luciferase Assay System (Promega, Cat \# N1111) according to manufacturer's protocols.

\section{In vivo thermal shift assay.}

Cells were harvested and suspended in PBS supplemented with $1 \mathrm{XPI}$ at $2 \times 10^{7} \mathrm{cells} / \mathrm{mL}$. The cells were treated with either DMSO or the indicated compound for $1 \mathrm{~h}$ at $37^{\circ} \mathrm{C}$. Each fraction $(90 \mu \mathrm{L})$ was incubated at the indicated temperature for $5 \mathrm{~min}$, followed by a 3 min incubation on ice. ${ }^{12}$ Cells were then lysed by addition of $10 \mu \mathrm{L} 10 \mathrm{X}$ cell lysis buffer with $50 \mathrm{X} \mathrm{PI}$ to a final concentration of $1 \mathrm{X}$ before the samples were centrifuged in a mini centrifuge at $21,000 \mathrm{~g}$ for 10 min at $4{ }^{\circ} \mathrm{C}$. Supernatants were separated from the pellet and collected for Western blot analysis.

\section{Photoaffinity labeling and enrichment of lenalidomide targets in living cells and on- bead digestion.}

Cells were plated on a 6-well plate and treated with the indicated compound for $1 \mathrm{~h}$ at $37^{\circ} \mathrm{C}$ before photoirradiation for $120 \mathrm{~s}$ at $4{ }^{\circ} \mathrm{C}$ via Dymax. After washing with PBS, cells were collected and lysed using $1 \mathrm{X}$ cell lysis buffer with $1 \mathrm{X} \mathrm{PI}$. The cleared lysates were then tagged with a cleavable biotin azide probe via CuAAC by addition of a pre-mixed cocktail (final concentrations: $100 \mu \mathrm{M}$ cleavable biotin azide probe, $100 \mu \mathrm{M}$ THPTA, $1 \mathrm{mM} \mathrm{CuSO}_{4}, 2 \mathrm{mM}$ freshly dissolved sodium ascorbate) and incubated for $3 \mathrm{~h}$ at $25{ }^{\circ} \mathrm{C}$ with inversion. The reactions were quenched by the addition of pre-chilled acetone and incubated for 20 min at $-20{ }^{\circ} \mathrm{C}$ to precipitate the proteins. Followed by centrifugation $\left(10 \mathrm{~min}, 4{ }^{\circ} \mathrm{C}, 21,300 \times \mathrm{g}\right)$, the protein was pelleted with supernatant discarded, and dried for 10 min at $25{ }^{\circ} \mathrm{C}$ to full dryness. The protein pellet was re-suspended in 1\% RapiGest/PBS by sonication ( $2 \mathrm{~s}$ on, $5 \mathrm{~s}$ off, $10 \%$ amplitude, $10 \mathrm{~s}$ total). The protein solutions were then diluted to the same concentrations after BCA assay, and $50 \mu \mathrm{L}$ of the solutions were taken and saved as input samples for Western blot analysis. Streptavidin beads slurry ( $80 \mu \mathrm{L}$ for each sample) was pre-washed three times with $400 \mu \mathrm{L}$ PBS in Bio-Spin columns before the protein solutions were added, followed by incubation for $16 \mathrm{~h}$ at $25{ }^{\circ} \mathrm{C}$ with inversion. The beads were then washed with $1 \mathrm{~mL}$ freshly dissolved $8 \mathrm{M}$ urea for three times, $1 \mathrm{~mL} 1 \%$ RapiGest/PBS for three times, and $1 \mathrm{~mL}$ PBS for three times. After the wash, $200 \mu \mathrm{L}$ PBS was added and $25 \mu \mathrm{L}$ of the bead slurry was taken out from the columns and saved as biotin enrichment samples for Western blot analysis. Rest of the bead slurry were subjected to on-bead digestion for mass spectrometry analysis.

In order to perform on-bead digestion, $25 \mu \mathrm{L}$ of $10 \mathrm{mM}$ DTT/PBS was added to each sample for 30 min at $25^{\circ} \mathrm{C}$ with inversion. The solution was discarded using the vacuum manifold and another $200 \mu \mathrm{L}$ of PBS was added together with $10 \mu \mathrm{L}$ of $500 \mathrm{mM}$ iodoacetamide/PBS to each Bio-Spin column to alkylate reduced cysteines for $30 \mathrm{~min}$ at $25^{\circ} \mathrm{C}$ with inversion. The solutions 
were removed using the vacuum manifold and the beads were washed with $1 \mathrm{~mL}$ PBS and 1 $\mathrm{mL} 50 \mathrm{mM}$ HEPES $(\mathrm{pH}=8.5)$ for one time before $100 \mu \mathrm{L} 0.5 \mathrm{M}$ guanidinium chloride $(\mathrm{GdnHCl})$ in $50 \mathrm{mM}$ HEPES ( $\mathrm{pH}=8.5$ ) was added into each column. Proteins on the beads were then digested by adding sequence-grade trypsin $(3 \mu \mathrm{g}, 6 \mu \mathrm{L}$ of a $0.5 \mathrm{mg} / \mathrm{mL}$ stock solution) for 16 $\mathrm{h}$ at $37{ }^{\circ} \mathrm{C}$ with inversion. The flow-through of the Bio-Spin columns after centrifugation containing the digested peptides were collected. The beads were washed with $200 \mu \mathrm{L} 0.5 \mathrm{M}$ $\mathrm{GdnHCl}$ in $50 \mathrm{mM} \mathrm{HEPES}$ once and $200 \mu \mathrm{L} 50 \%$ acetonitrile/ water once with the flow-through all combined with the digested peptides. The eluted peptides were then dried on the SpeedVac to dryness and subjected to mass spectrometry sample preparation.

\section{Quantitative mass spectrometry sample preparation.}

Digested peptides were resuspended in $50 \mu \mathrm{L}$ of $50 \mathrm{mM} \mathrm{TEAB}$ buffer $(\mathrm{pH}=8.5)$, treated with $5 \mu \mathrm{L}$ of the TMT 16-plex reagents $(10 \mu \mathrm{g} / \mu \mathrm{L})$ and then incubated for $1 \mathrm{~h}$ at $25^{\circ} \mathrm{C}$. The TMT labeling was quenched with $2 \mu \mathrm{L}$ of $5 \%$ hydroxylamine solution for $15 \mathrm{~min}$ at $25^{\circ} \mathrm{C}$. The TMTlabeled peptides were then combined and dried on the Speed-Vac. The peptides were

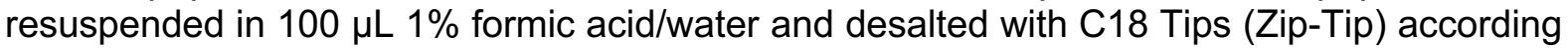
to the manufacturer's instructions and stored at $-20{ }^{\circ} \mathrm{C}$ until analysis.

\section{ERLIC separation and mass spectrometry analysis.}

The sample was separated using the Pierce ${ }^{\mathrm{TM}}$ High $\mathrm{pH}$ Reversed-Phase Peptide Fractionation Kit (Thermo Fischer) according to manufacturer's instructions. After separation, each fraction was submitted for a single LC-MS/MS experiment that was performed on a Lumos Tribrid Orbitrap equipped with an UltiMate 3000 RSLCnano System. Peptides were separated onto a $100 \mu \mathrm{m}$ inner diameter microcapillary trapping column packed first with approximately $3 \mathrm{~cm}$ of $\mathrm{C} 18$ Reprosil resin $(5 \mu \mathrm{m}, 100 \AA$, Dr. Maisch $\mathrm{GmbH}$, Germany) followed by analytical column $50 \mathrm{~cm}$ microcapillary based PharmaFluidics (Belgium). Separation was achieved through applying a gradient from $5-27 \%$ acetonitrile in $0.1 \%$ formic acid in water

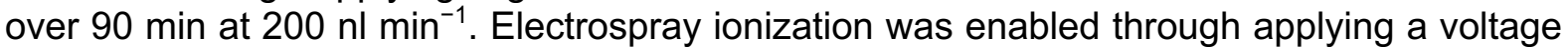
of $1.8 \mathrm{kV}$ using a home-made electrode junction at the end of the microcapillary column and sprayed from stainless-steel needle (PepSep, Denmark). The Lumos Orbitrap was operated in data-dependent mode for the mass spectrometry methods. The mass spectrometry survey scan was performed in the Orbitrap in the range of $400-1,800 \mathrm{~m} / \mathrm{z}$ at a resolution of $1.2 \times 10^{5}$, followed by the selection of the 10 most intense ions (TOP10) for CID-MS2 fragmentation in the lon trap using a precursor isolation width window of $2 \mathrm{~m} / \mathrm{z}$, an AGC setting of 10,000 , and a maximum ion accumulation of $200 \mathrm{~ms}$. Singly charged ion species were not subjected to CID fragmentation. Normalized collision energy was set to $35 \mathrm{~V}$ and an activation time of 10 $\mathrm{ms}$. Ions in a 10-ppm $\mathrm{m} / \mathrm{z}$ window around ions selected for MS2 were excluded from further selection for fragmentation for $60 \mathrm{~s}$. The same TOP10 ions were subjected to HCD MS2 event in Orbitrap part of the instrument. The fragment ion isolation width was set to $0.8 \mathrm{Da}$, with 0.3 Da offset, AGC was set to 50,000 , the maximum ion time was $100 \mathrm{~ms}$, normalized collision energy was set to $34 \mathrm{~V}$ and an activation time of $1 \mathrm{~ms}$ for each HCD MS2 scan.

\section{Quantitative mass spectrometry data analysis.}

The raw data were analyzed using Proteome Discoverer 2.4.1.15. Assignment of MS/MS spectra was performed using the Sequest $\mathrm{HT}$ algorithm by searching the data against a protein sequence database including all entries from the Human Uniprot database (Aug 19, 2016, 20,156 SwissProt) and a list of common contaminant proteins. Search parameters included: a 10-ppm precursor ion tolerance and 0.02 Da fragment ion tolerance for HCD or 0.6 Da fragment ion tolerance for CID, full tryptic protease specificity with up to two missed cleavages, a static modification of 16-plex TMTpro tags on peptide $\mathrm{N}$-termini and lysine residues $(+304.2071 \mathrm{Da})$, a static modification of cysteine carbamidomethylation (+57.0214 Da), a dynamic modification of methionine oxidation (+15.9949 Da), a dynamic modification of 
deamidation on asparagine and glutamine residues $(+0.9847 \mathrm{Da})$ and a dynamic modification of acetylation on peptide N-termini $(+42.0106 \mathrm{Da})$. Peptide spectral matches (PSMs) were filtered for a false discovery rate (FDR) of $1 \%$ following a target-decoy analysis with Percolator. Reporter ions were quantified using a $0.02 \mathrm{~m} / \mathrm{z}$ window centered on the theoretical $\mathrm{m} / \mathrm{z}$ value and the intensity of the signal closest to the theoretical $\mathrm{m} / \mathrm{z}$ value was recorded. Reporter ion intensities were exported in result file of Proteome Discoverer search engine as an excel tables. The total signal intensity across all peptides quantified was summed and normalized for each TMT channel. Quantified proteins were required to have at least three unique peptides and four separate PSMs. All TMT-based experiments were performed with four replicates.

\section{In vivo ubiquitylation assay.}

In vivo ubiquitylation assay was performed according to the method of Jaffrey and coworkers. ${ }^{13}$ HEK293T or HEK293T CRBN-KD cells ${ }^{14}$ transiently transfected with HA-Ub were treated with either DMSO or the indicated compound for $1 \mathrm{~h}$ before $10 \mu \mathrm{M}$ MG132 was added. Treated cells were incubated for $6 \mathrm{~h}$ at $37{ }^{\circ} \mathrm{C}$ and collected. Ubiquitylated proteins were immunoprecipitated by the indicated antibody according to the manufacturer's instructions. Samples were then eluted and subjected to Western blot analysis.

\section{Software.}

Data were analyzed and visualized using Microsoft Excel (v16.22) and GraphPad Prism (v8.0.1), in addition to software listed by experiment. NMR data were analyzed using MestReNova (v14.0.1). DNA and protein sequences were analyzed using Geneious (v10.0.7). FACS data were analyzed by FlowJo (v10.6.1). Proteomics data were analyzed by Proteome Discoverer (v2.4.1.15). Images were made using ImageJ $(\mathrm{NIH}, \mathrm{v} 1.50 \mathrm{i})$, ImageStudioLite (v5.2.5), and Adobe Illustrator (v22.1). Docking studies were performed with Molecular Operating Environment (MOE, v2020.09) and the obtained structures were analyzed with PyMOL (v2.3.1).

\section{Statistical analysis.}

Statistical analyses (unpaired Student's $t$-tests) were performed using GraphPad Prism. Data were derived from at least three biological replicate experiments and presented as the mean \pm s.d., ${ }^{*} P<0.0332,{ }^{* *} P<0.0021,{ }^{* * *} P<0.0002,{ }^{* * * *} P<0.0001$ and n.s., not significant. 


\section{REFERENCES}

1. Still, W. C.; Kahn, M.; Mitra, A., Rapid chromatographic technique for preparative separations with moderate resolution. J. Org. Chem. 1978, 43 (14), 2923-2925.

2. Li, Z.; Hao, P.; Li, L.; Tan, C. Y.; Cheng, X.; Chen, G. Y.; Sze, S. K.; Shen, H. M.; Yao, S. Q., Design and synthesis of minimalist terminal Alkyne-Containing diazirine photo-crosslinkers and their incorporation into kinase inhibitors for cell-and tissue-based proteome profiling. Angew. Chem. Int. Ed. 2013, 125 (33), 8713-8718. 3. Gao, J.; Mfuh, A.; Amako, Y.; Woo, C. M., Small molecule interactome mapping by photoaffinity labeling reveals binding site hotspots for the NSAIDs. J. Am. Chem. Soc. 2018, 140 (12), 4259-4268.

4. Miyamoto, D. K.; Flaxman, H. A.; Wu, H. Y.; Gao, J.; Woo, C. M., Discovery of a Celecoxib Binding Site on Prostaglandin E Synthase (PTGES) with a Cleavable Chelation-Assisted Biotin Probe. ACS Chem. Biol. 2019, 14 (12), 2527-2532.

5. Pangborn, A. B.; Giardello, M. A.; Grubbs, R. H.; Rosen, R. K.; Timmers, F. J., Safe and convenient procedure for solvent purification. Organometallics 1996, 15 (5), 1518-1520.

6. Muller, G. W.; Chen, R.; Huang, S.-Y.; Corral, L. G.; Wong, L. M.; Patterson, R. T.; Chen, Y.; Kaplan, G.; Stirling, D. I., Amino-substituted thalidomide analogs: potent inhibitors of TNF-a production. Bioorganic Med. Chemi. Lett. 1999, 9 (11), 1625-1630.

7. Kleiner, P.; Heydenreuter, W.; Stahl, M.; Korotkov, V. S.; Sieber, S. A., A whole proteome inventory of background photocrosslinker binding. Angew. Chem. Int. Ed. 2017, 56 (5), 1396-1401.

8. $\quad$ Matyskiela, M. E.; Lu, G.; Ito, T.; Pagarigan, B.; Lu, C.-C.; Miller, K.; Fang, W.; Wang, N.-Y.; Nguyen, D.; Houston, J., A novel cereblon modulator recruits GSPT1 to the CRL4 CRBN ubiquitin ligase. Nature 2016, 535 (7611), 252-257.

9. Sebej, P.; Lim, B. H.; Park, B. S.; Givens, R. S.; Klán, P., The power of solvent in altering the course of photorearrangements. Org. Lett. 2011, 13 (4), 644-647.

10. Man, H.-W.; Muller, G. W.; Ruchelman, A. L.; Khalil, E. M.; Chen, R. S.-C.; Zhang, W. Arylmethoxy isoindoline derivatives and compositions comprising and methods of using the same. US2011/0196150A1, 2011. 11. Sievers, Q. L. P., G., Bunker, R. D.; Renneville, A.; Slabicki, M.; Liddicoat, B. J.; Abdulrahman, W.; Mikkelsen, T.; Ebert, B. L.; Thoma, N. H., Defining the human $\mathrm{C} 2 \mathrm{H} 2$ zinc finger degrome targeted by thalidomide analogs through CRBN. Science 2018, 362 (6414), eaat0572.

12. de Wispelaere, M.; Du, G.; Donovan, K. A.; Zhang, T.; Eleuteri, N. A.; Yuan, J. C.; Kalabathula, J.; Nowak, R. P.; Fischer, E. S.; Gray, N. S.; Yang, P. L., Small molecule degraders of the hepatitis C virus protease reduce susceptibility to resistance mutations. Nat. Commun. 2019, 10 (1), 3468.

13. Xu, G.; Jiang, X.; Jaffrey, S. R., A mental retardation-linked nonsense mutation in cereblon is rescued by proteasome inhibition. J. Biol. Chem. 2013, 288 (41), 29573-85.

14. Nguyen, T. V.; Lee, J. E.; Sweredoski, M. J.; Yang, S. J.; Jeon, S. J.; Harrison, J. S.; Yim, J. H.; Lee, S. G.; Handa, H.; Kuhlman, B.; Jeong, J. S.; Reitsma, J. M.; Park, C. S.; Hess, S.; Deshaies, R. J., Glutamine Triggers Acetylation-Dependent Degradation of Glutamine Synthetase via the Thalidomide Receptor Cereblon. Mol. Cell. 2016, 61 (6), 809-20. 


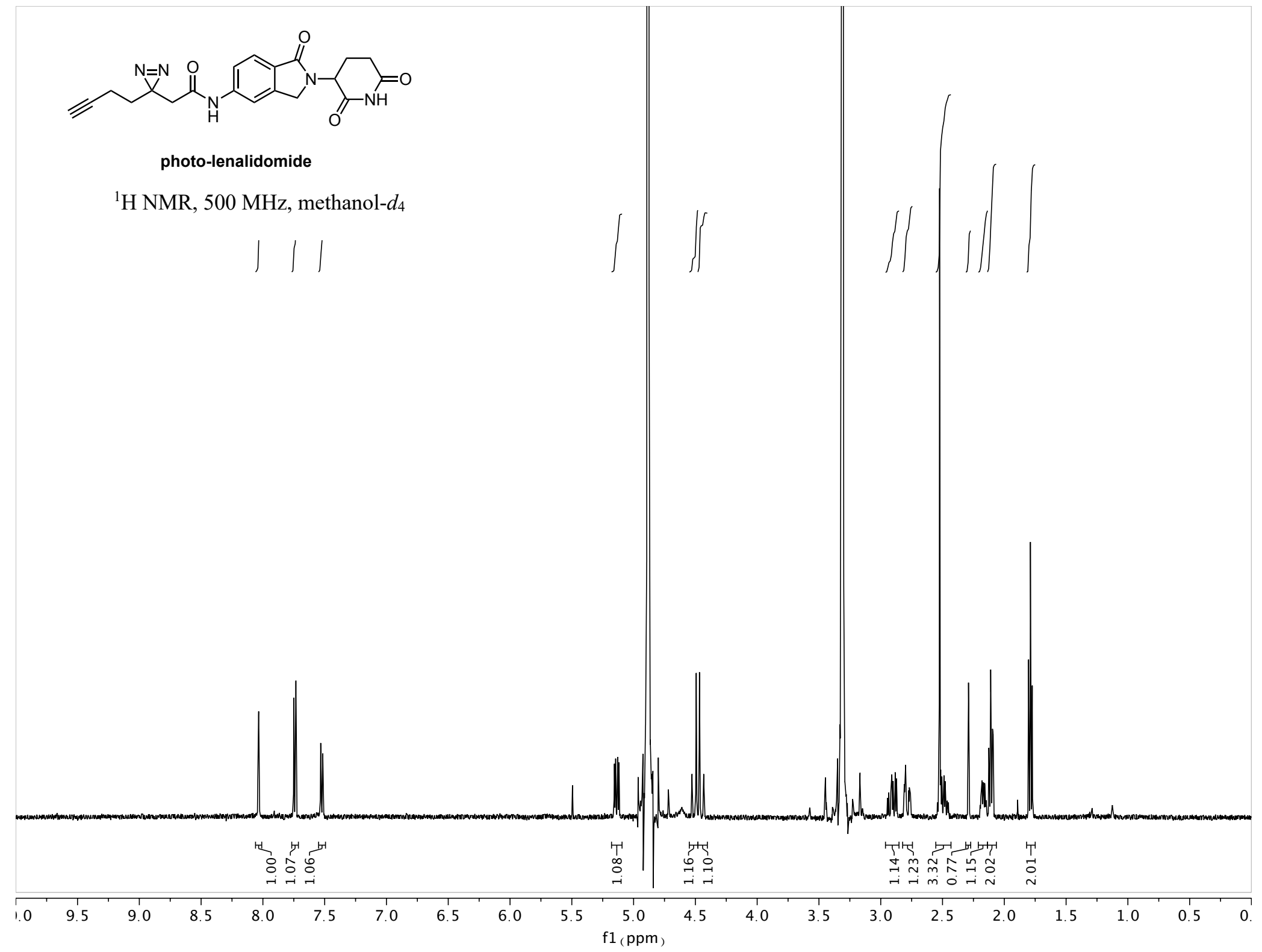




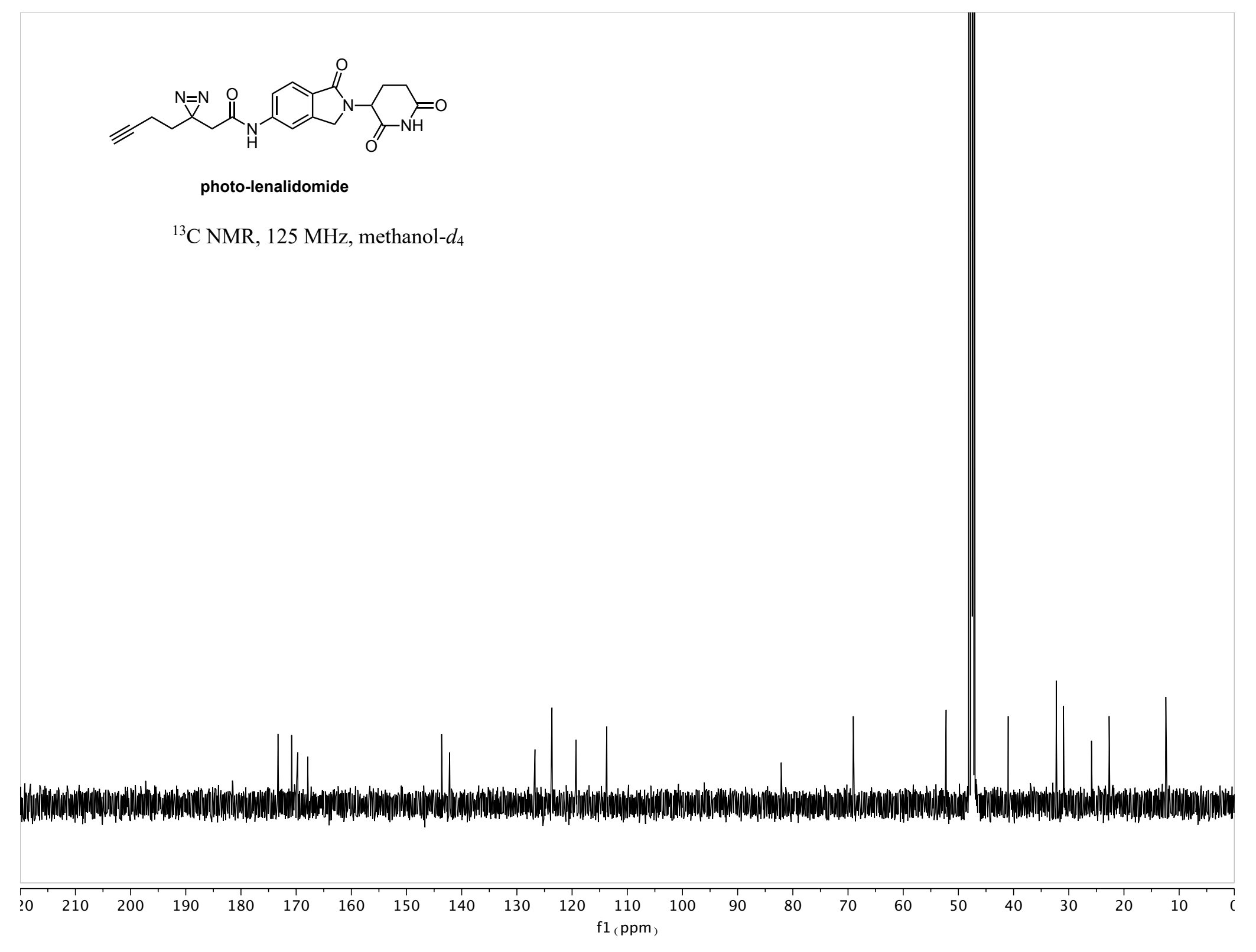



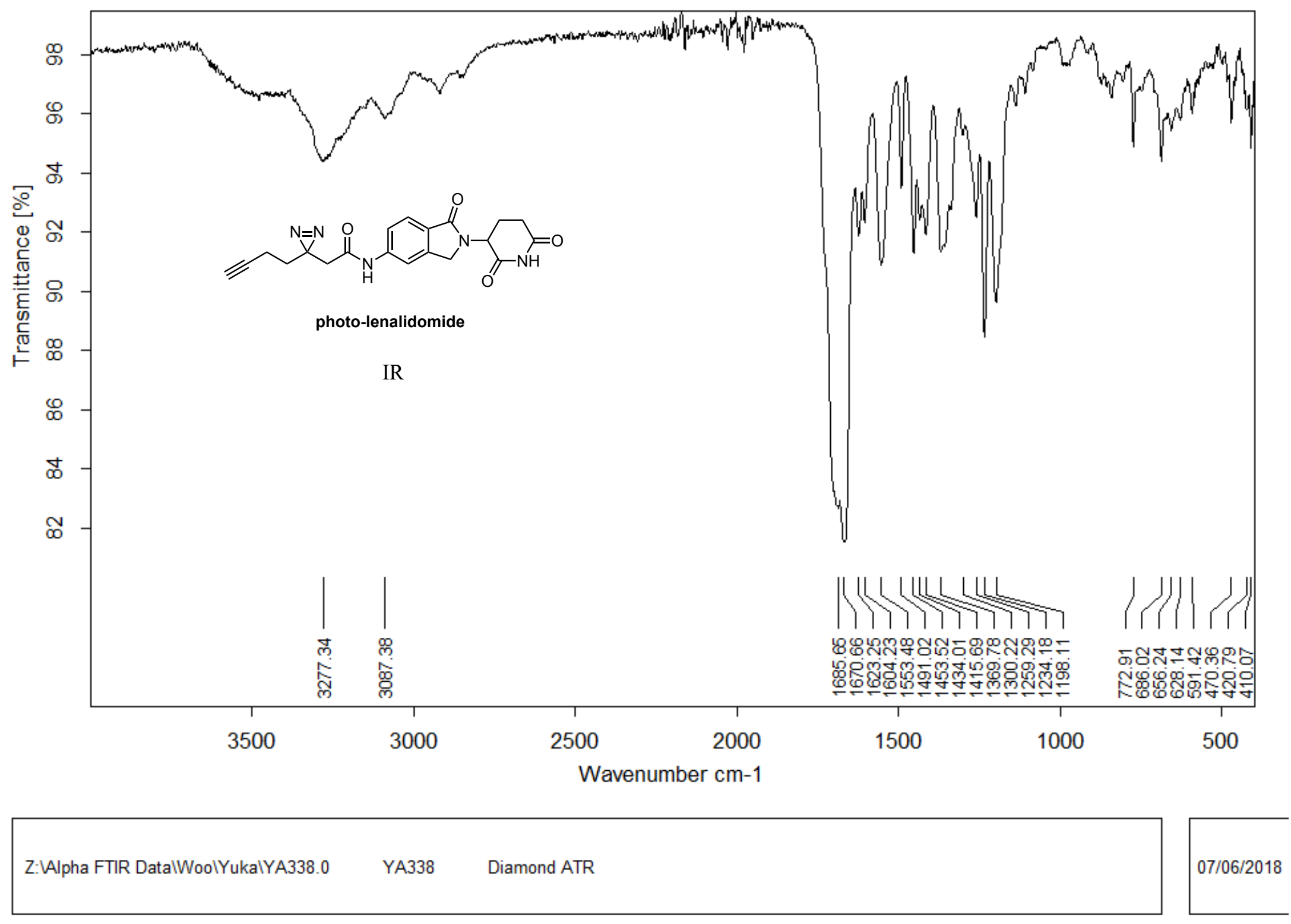

Page 1/2 


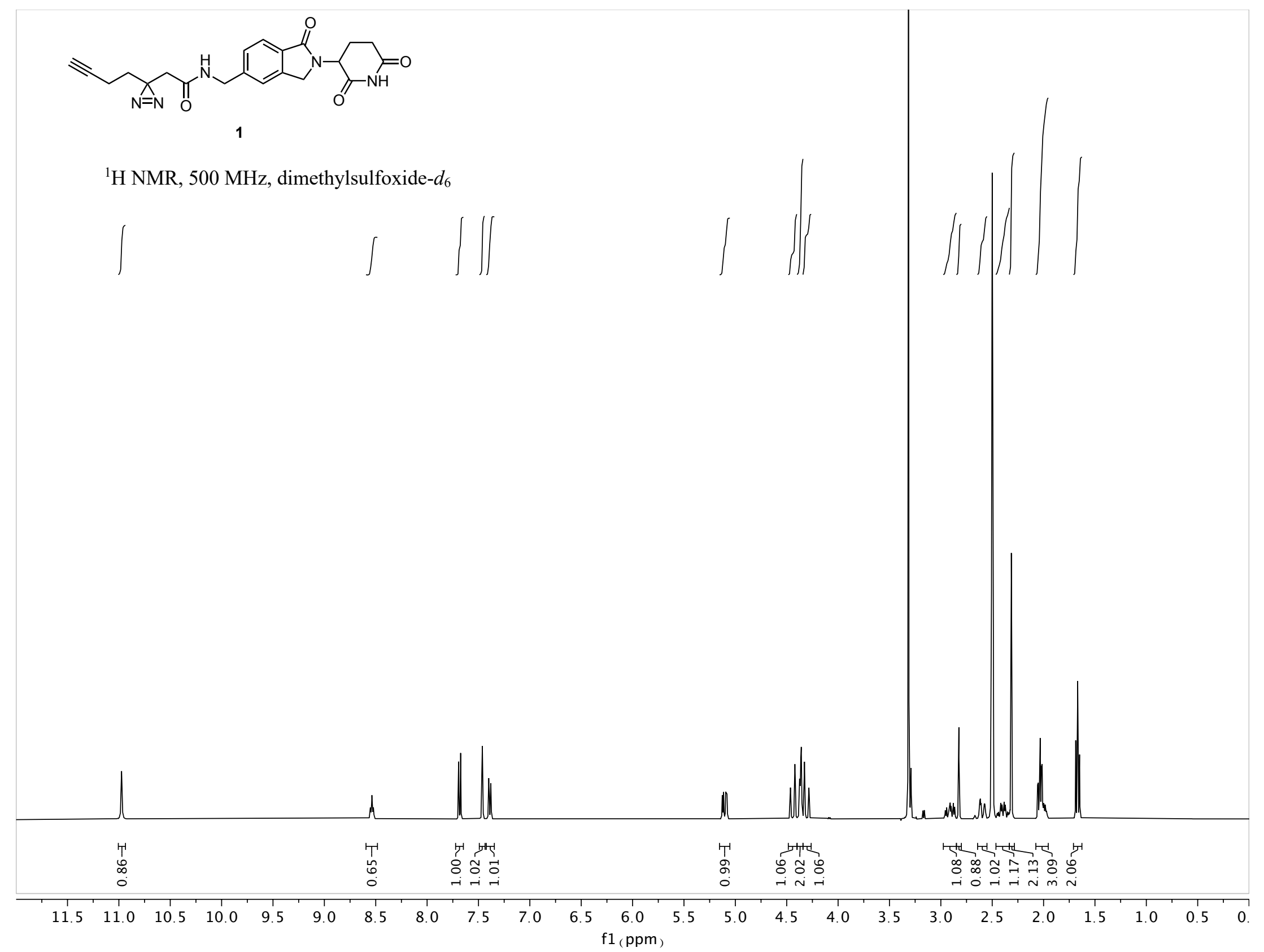




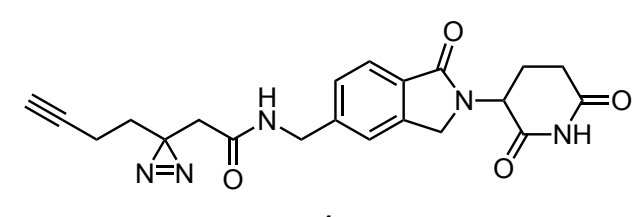

${ }^{13} \mathrm{C}$ NMR, $125 \mathrm{MHz}$, dimethylsulfoxide- $d_{6}$ 


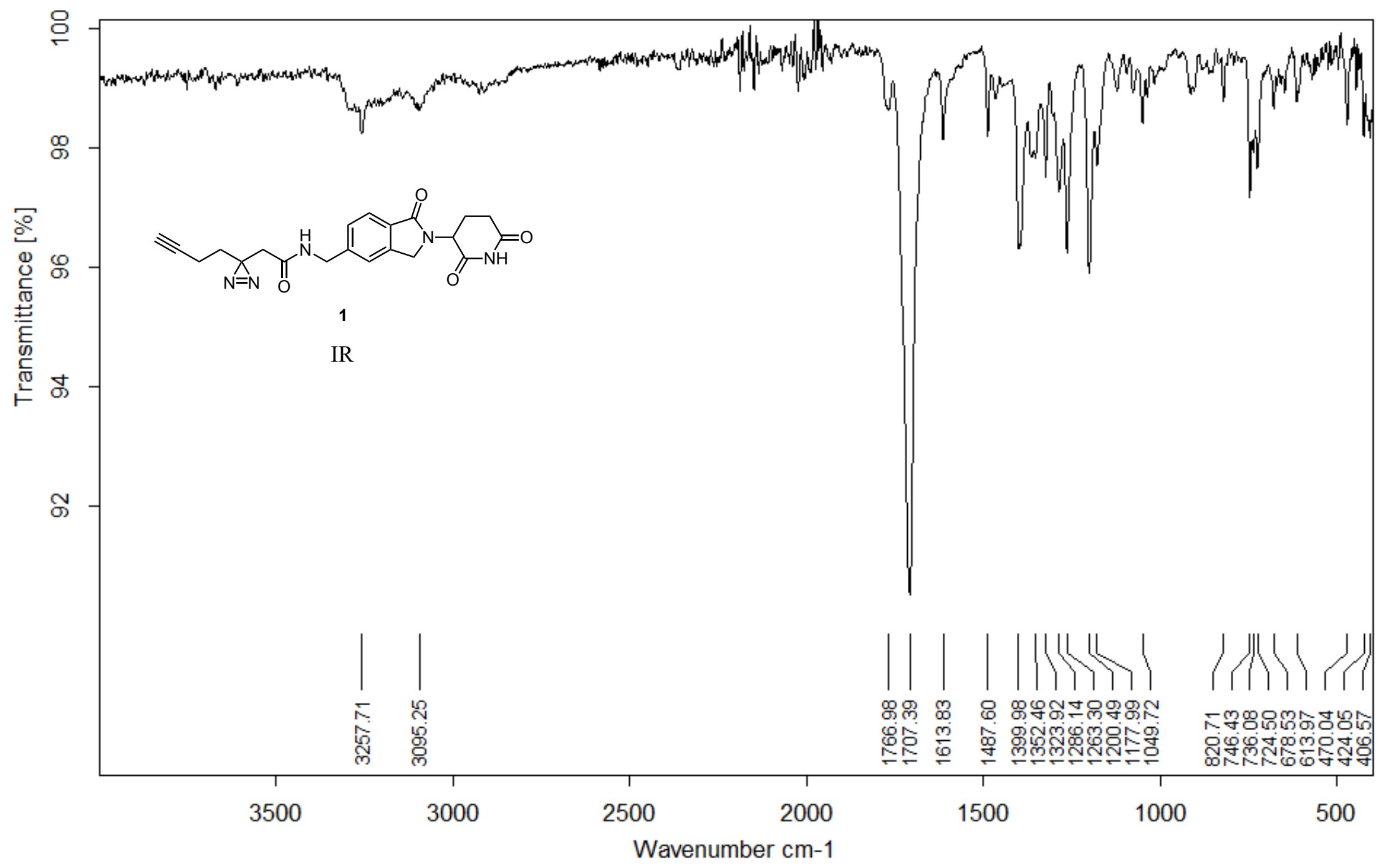

Z: Alpha FTIR DatalWoolYukalYA322.0 YA322 Diamond ATR 


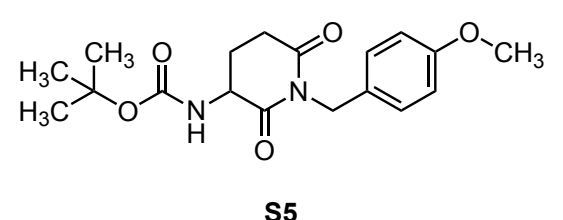

${ }^{1} \mathrm{H}$ NMR, $500 \mathrm{MHz}$, chloroform- $d$

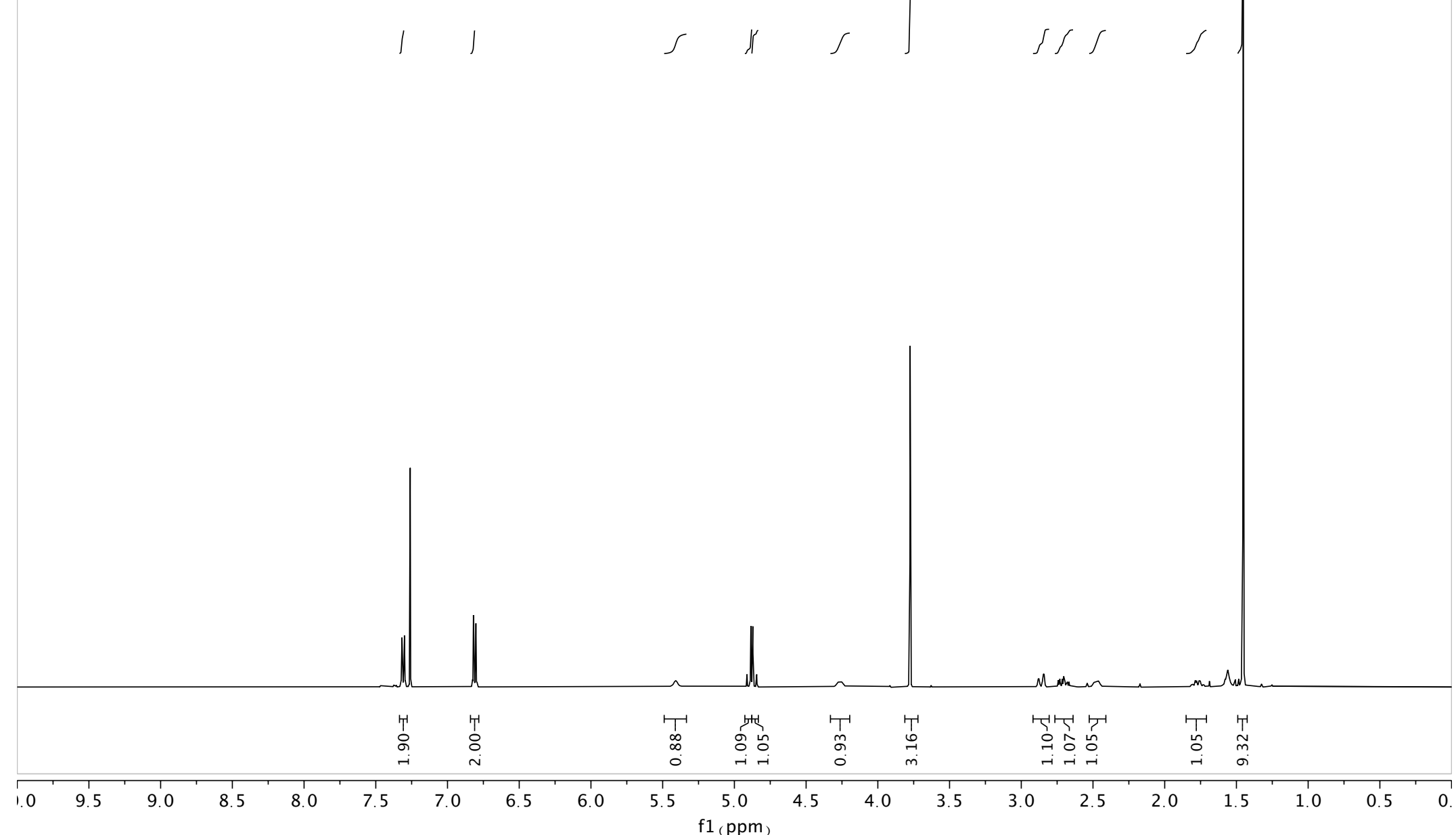




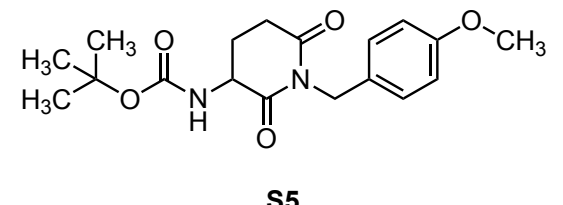

${ }^{13} \mathrm{C}$ NMR, $125 \mathrm{MHz}$, chloroform- $d$

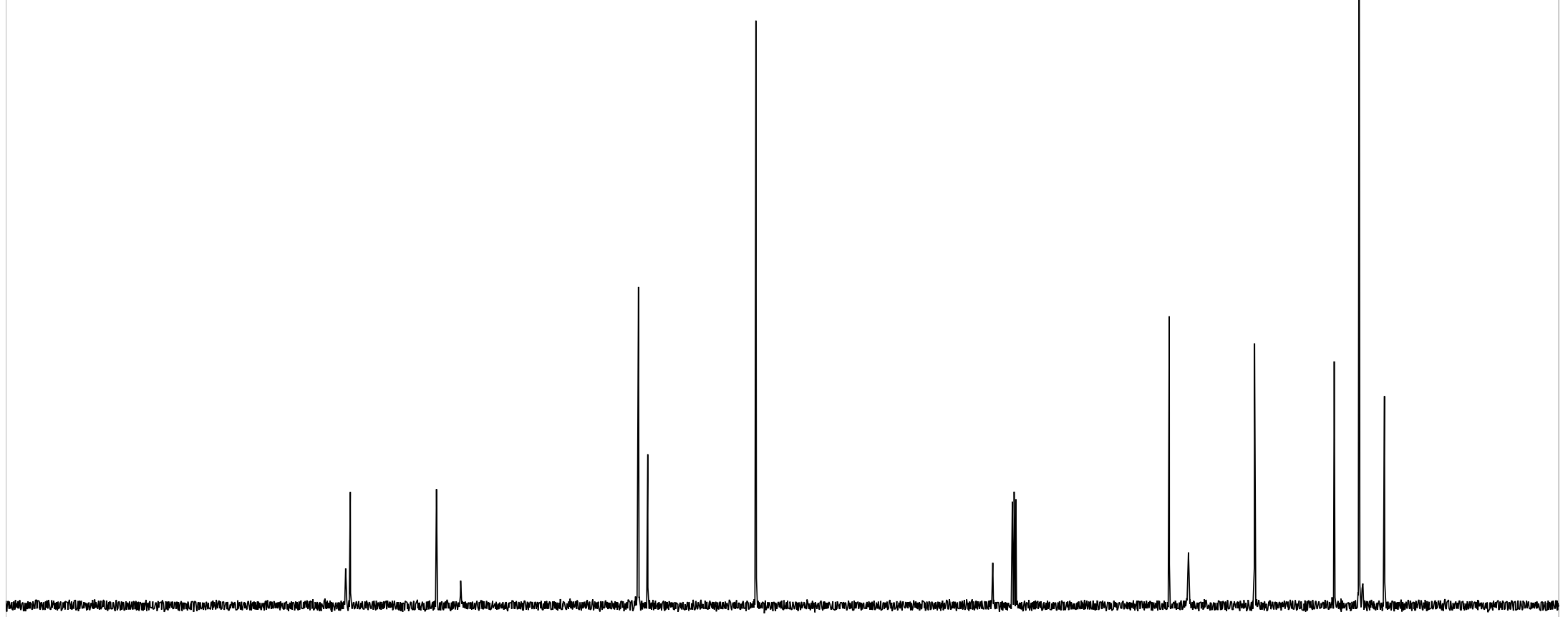




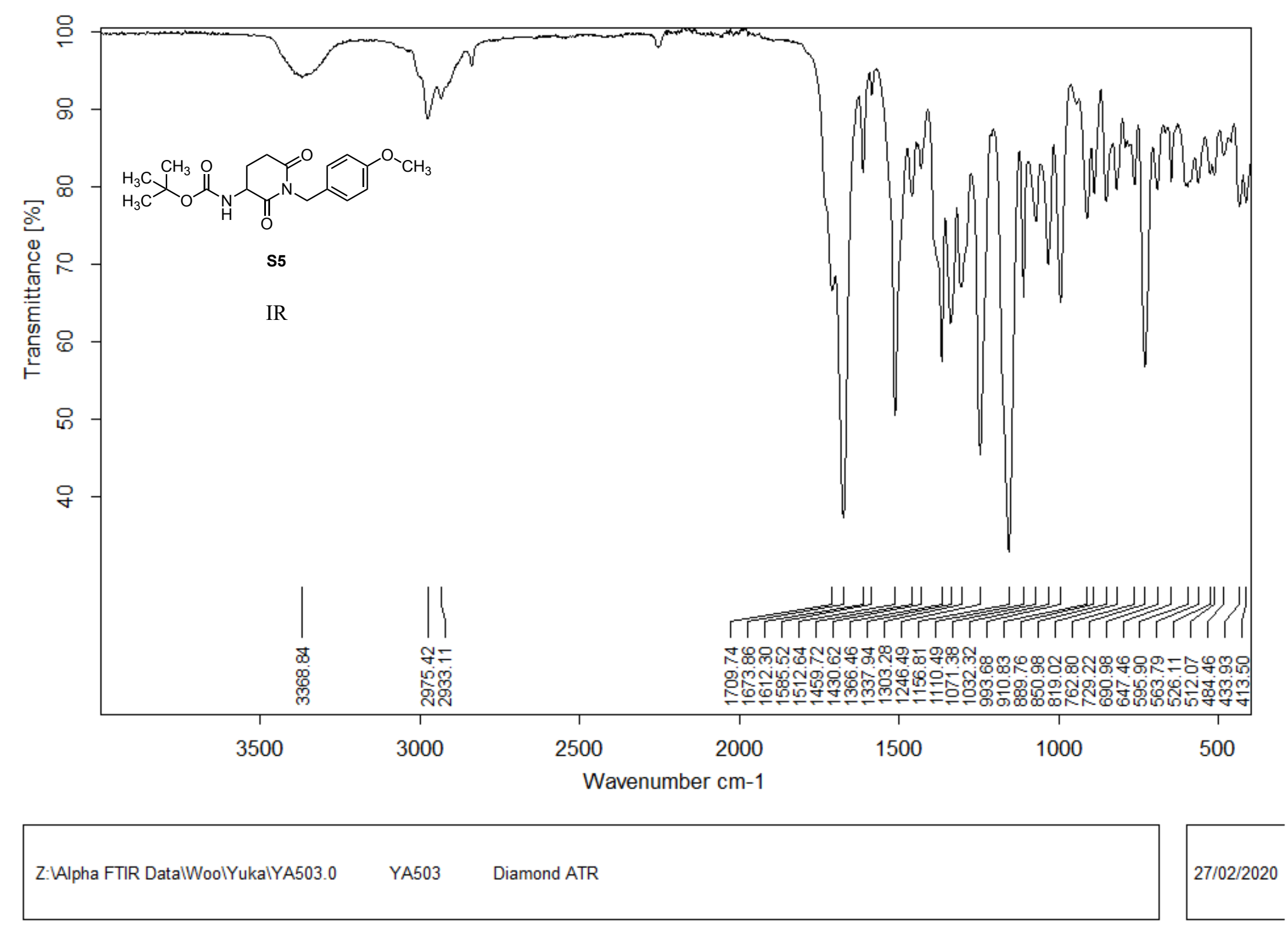

Page 1/2 


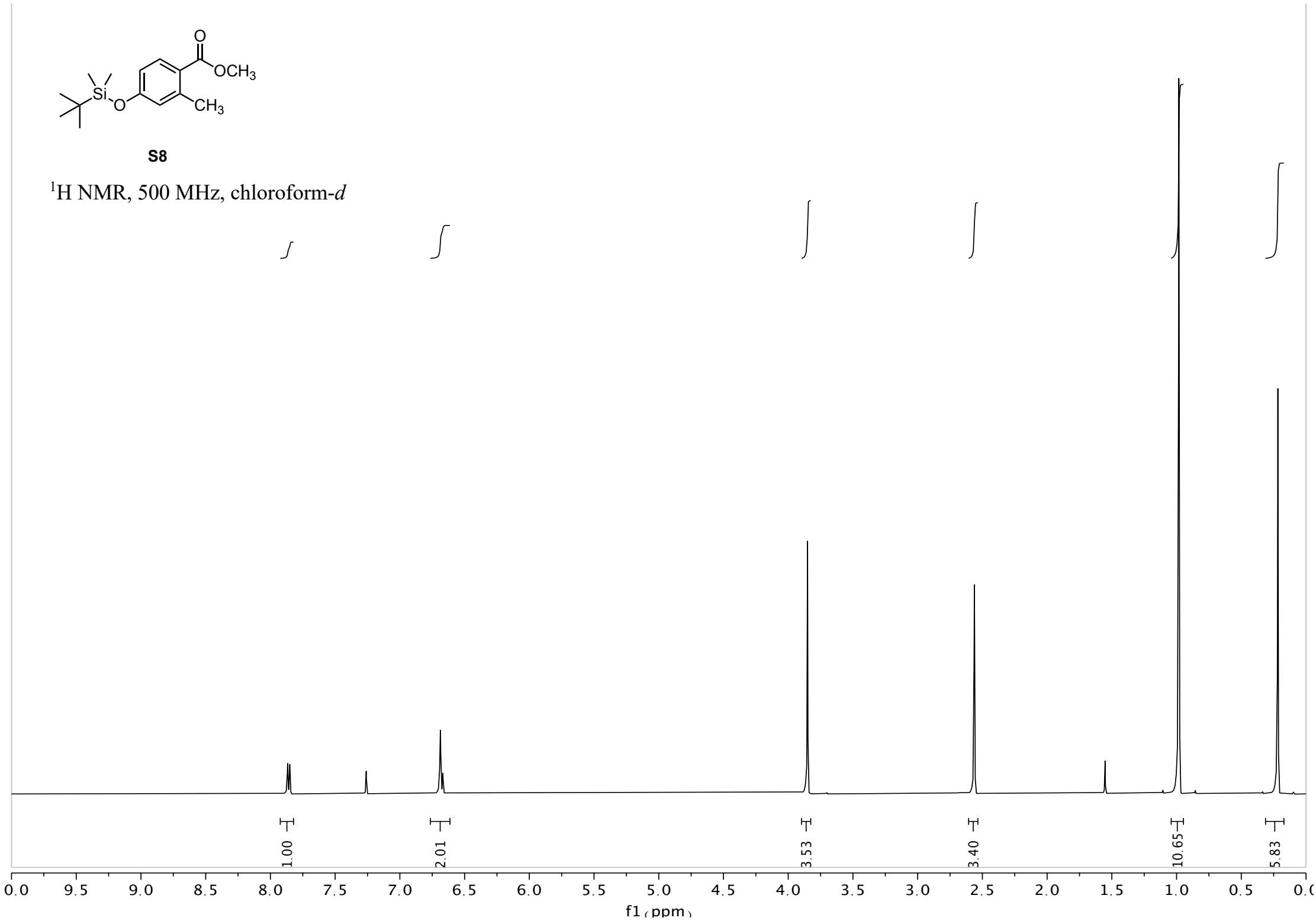




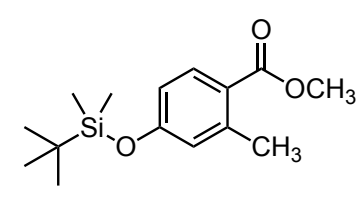

S8

${ }^{13} \mathrm{C}$ NMR, $125 \mathrm{MHz}$, chloroform-d

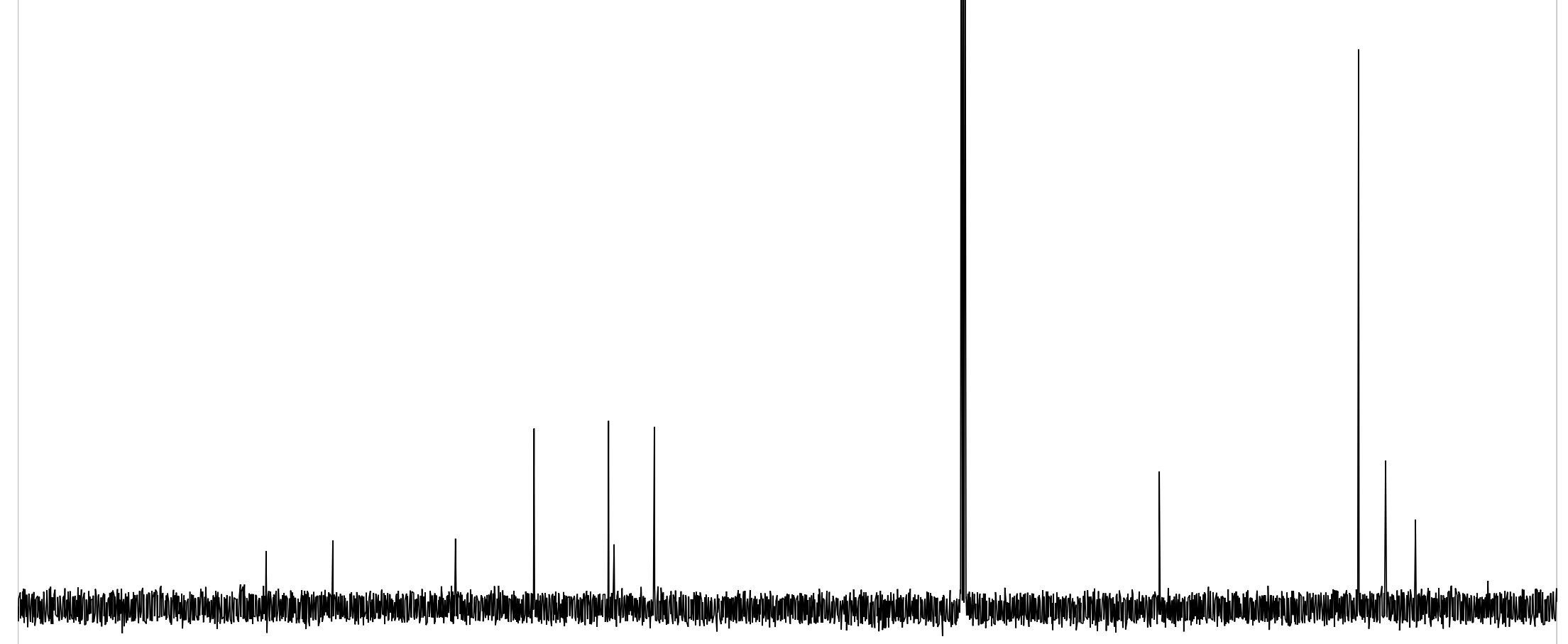

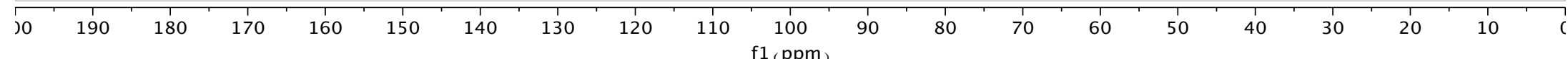






Z:VALPHA FTIR DATAIWoolYukalYA513.0 YA513 Diamond ATR 


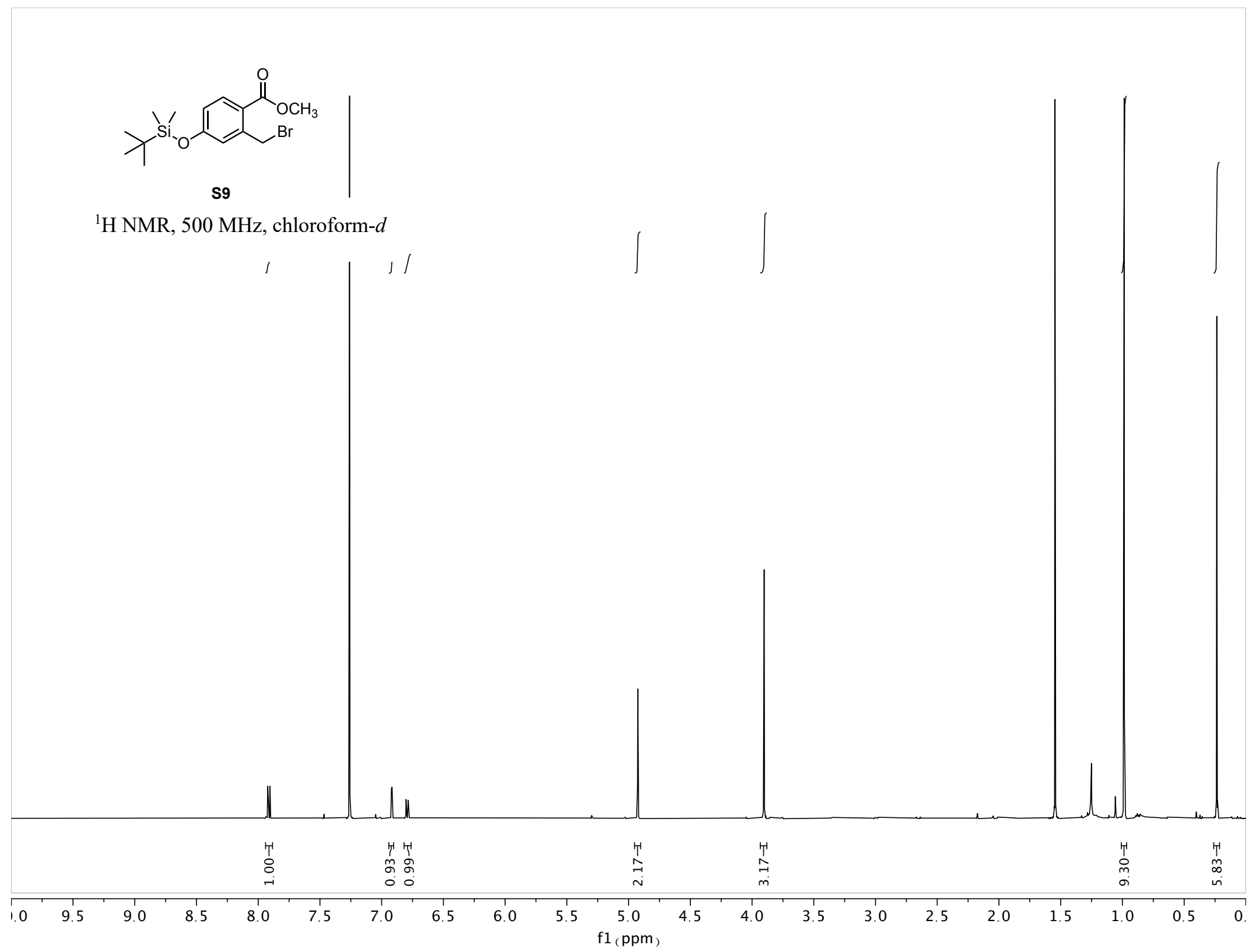

S58 


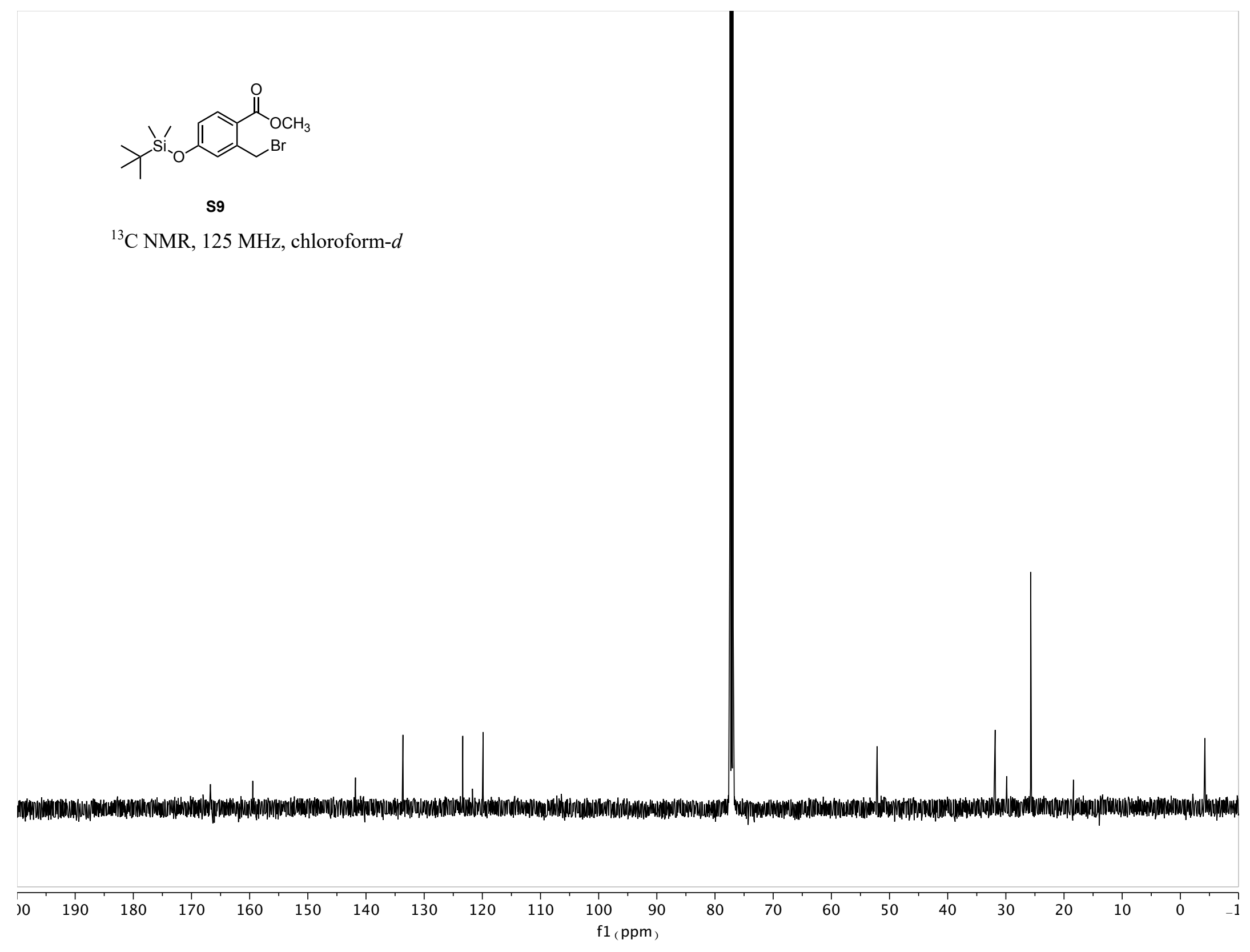




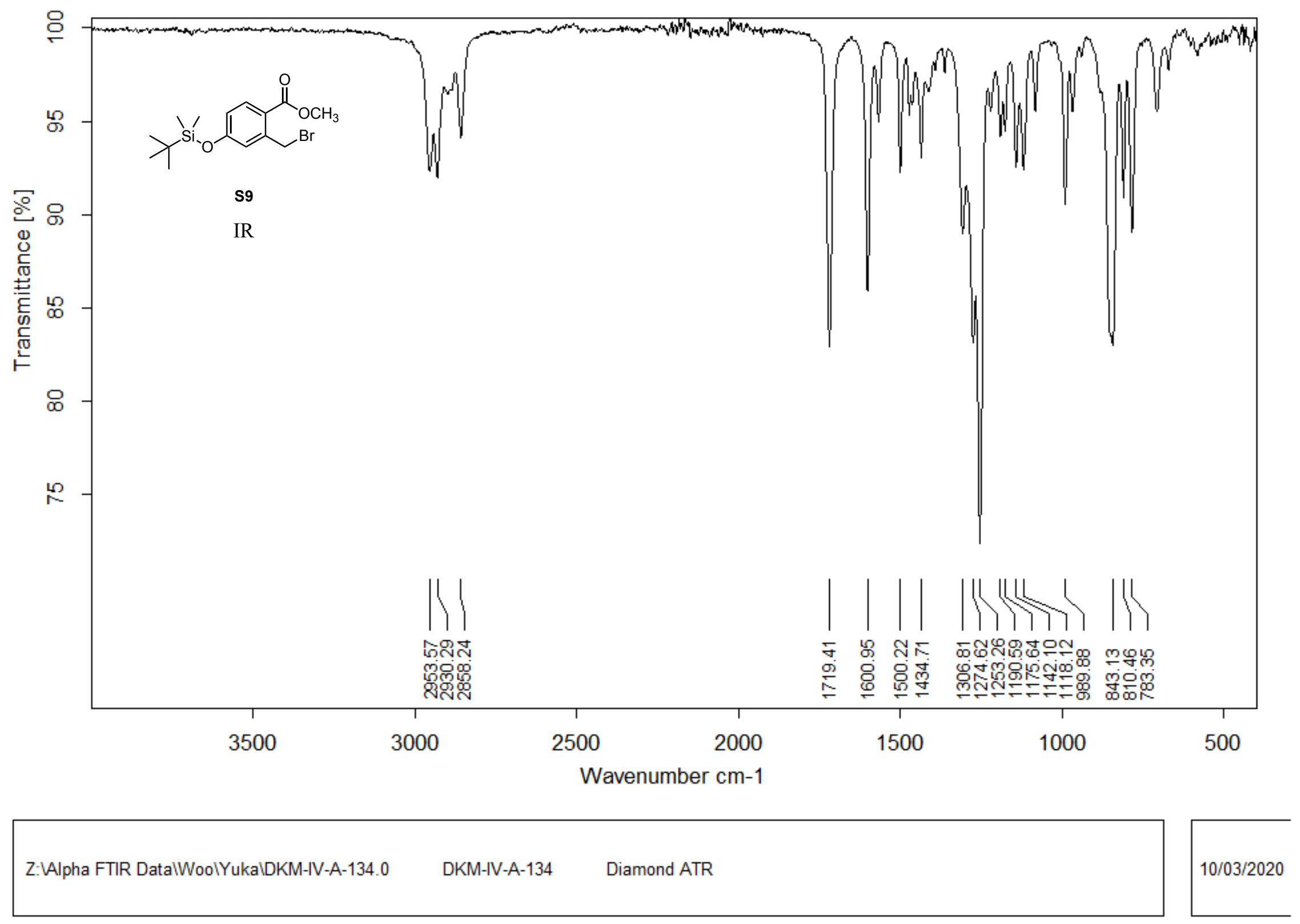

Page 1/2 


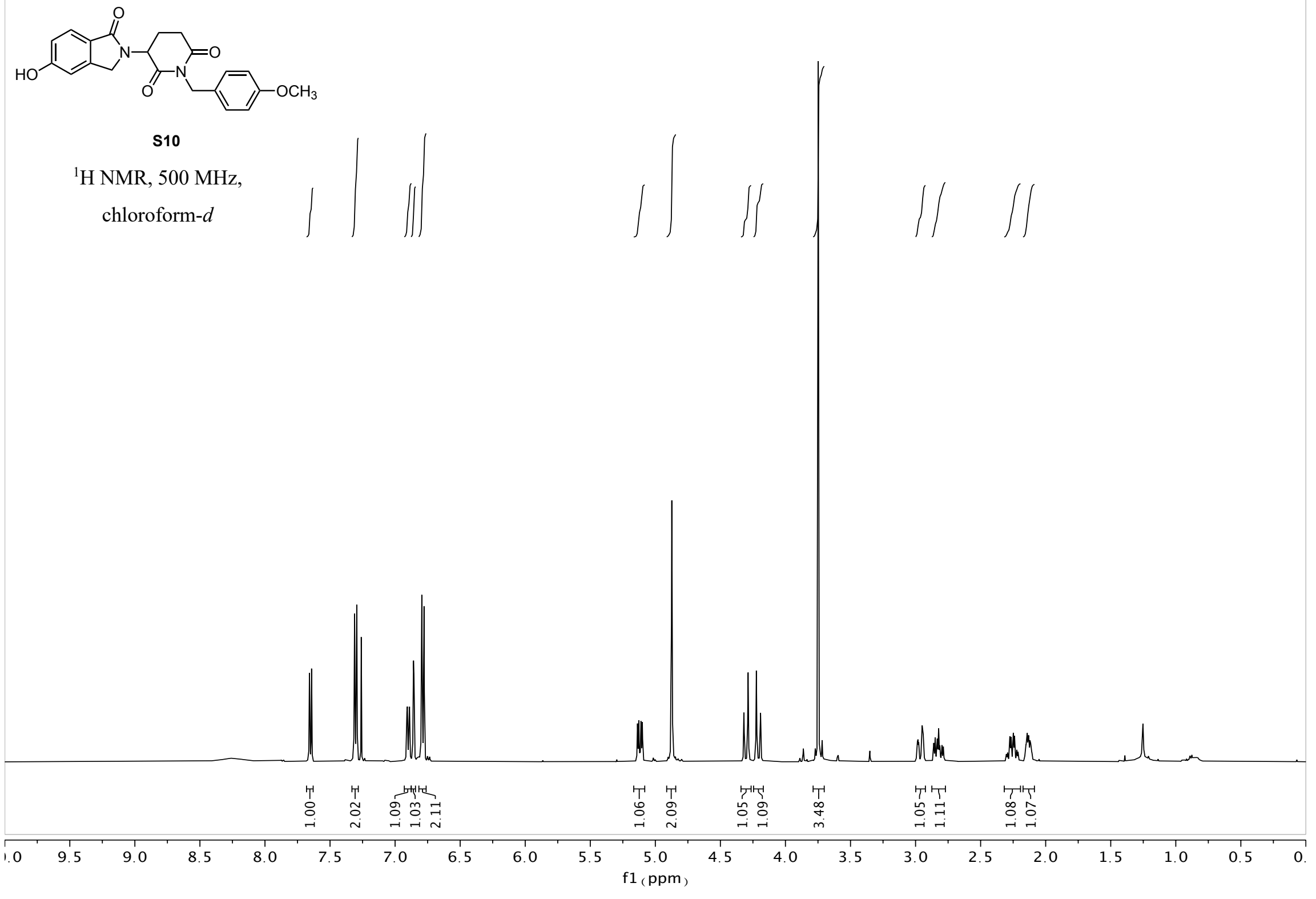




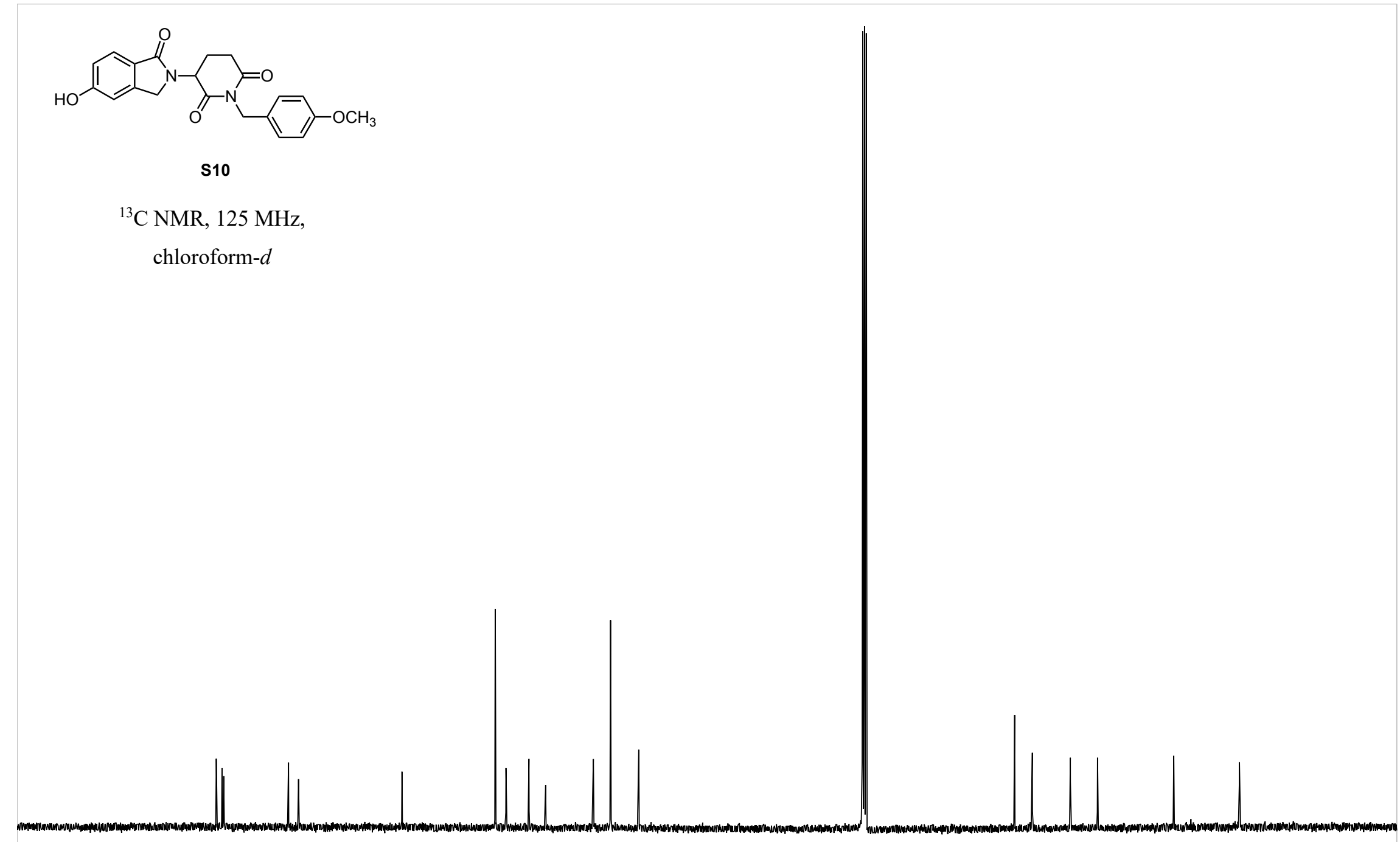

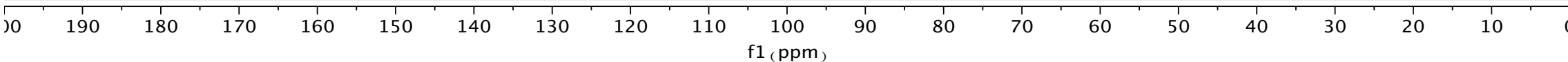




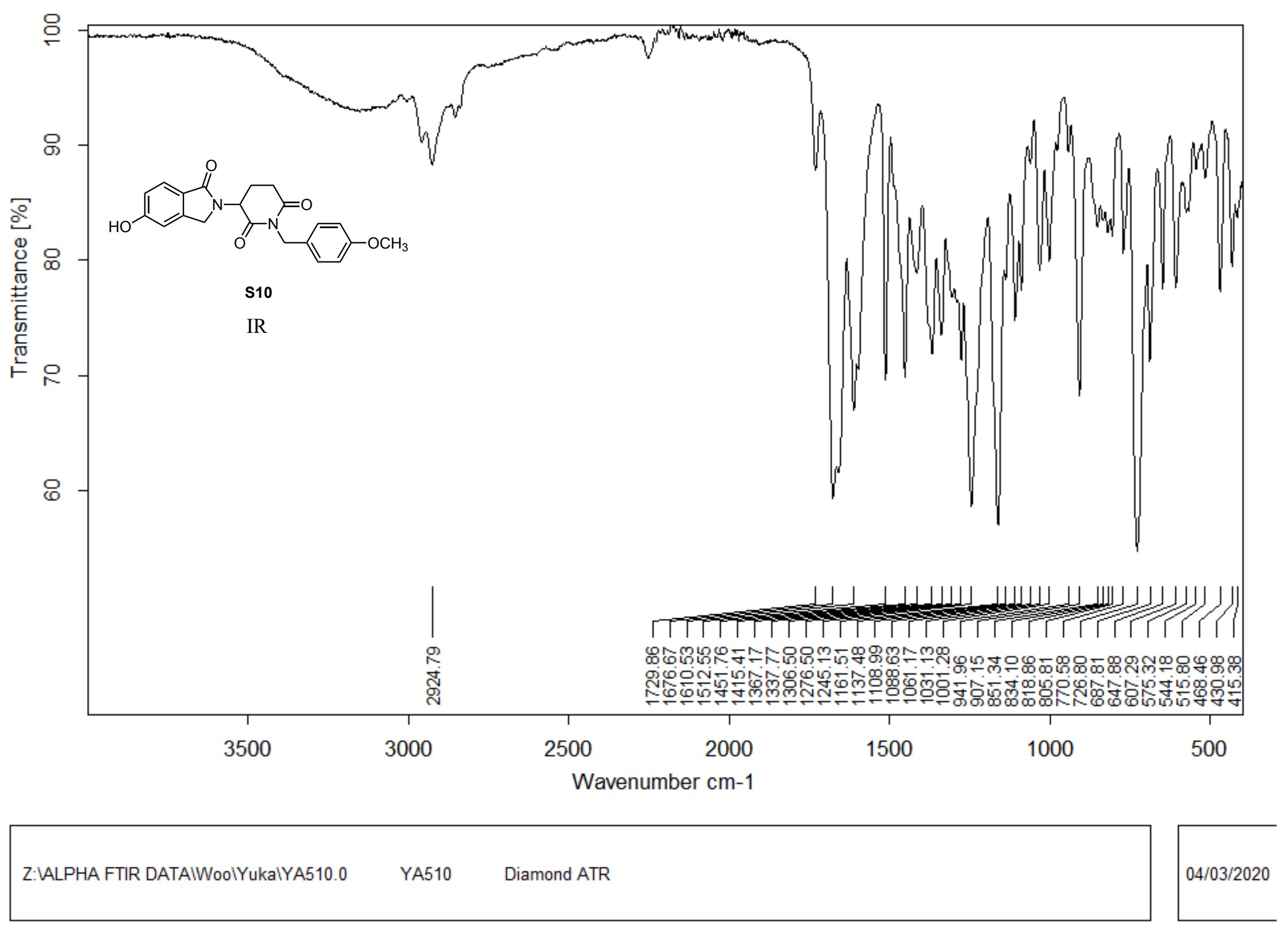

Page 1/2 


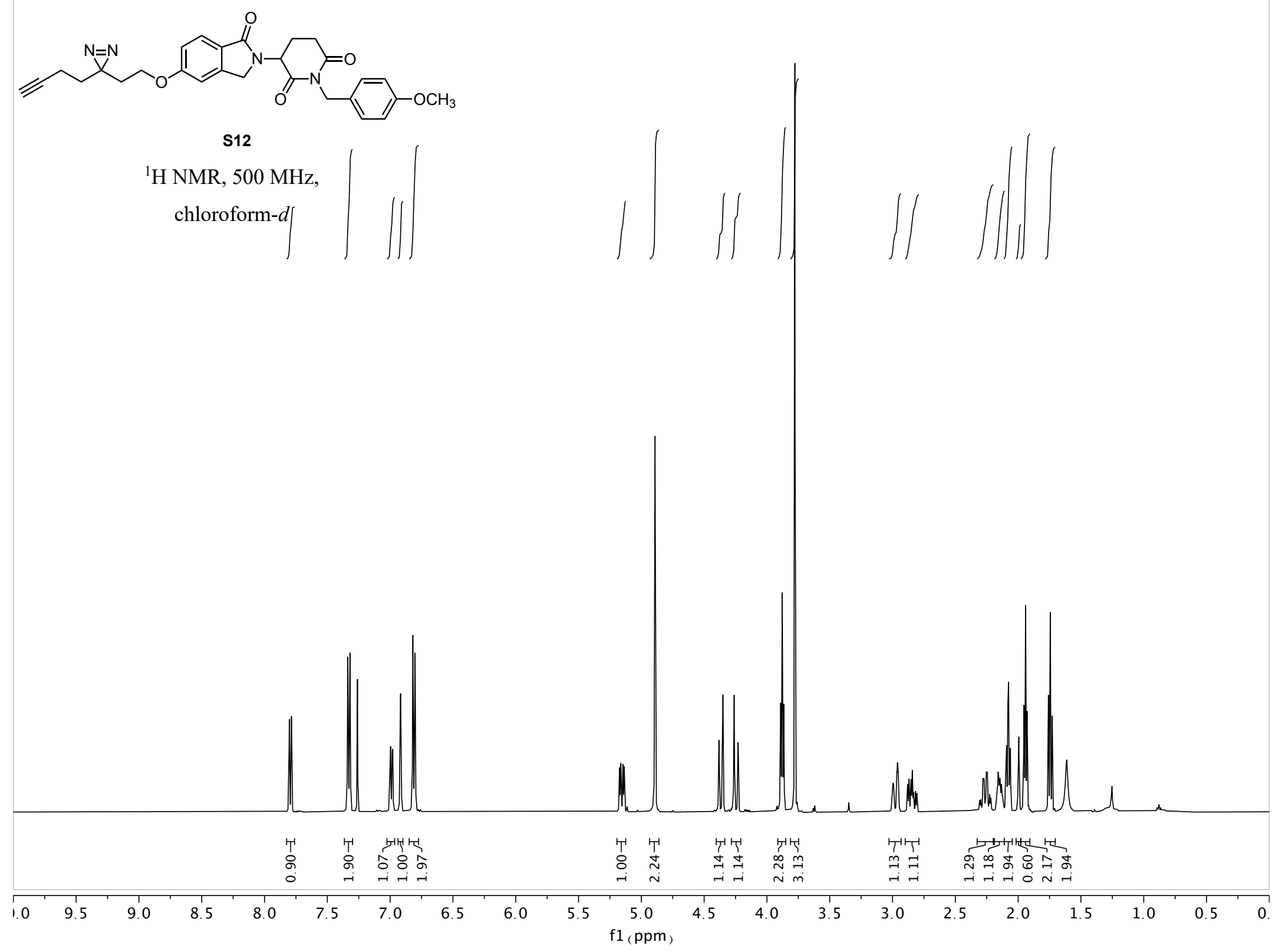




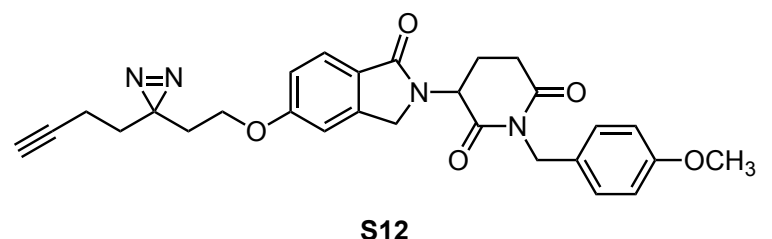

${ }^{13} \mathrm{C}$ NMR, $125 \mathrm{MHz}$,

chloroform- $d$

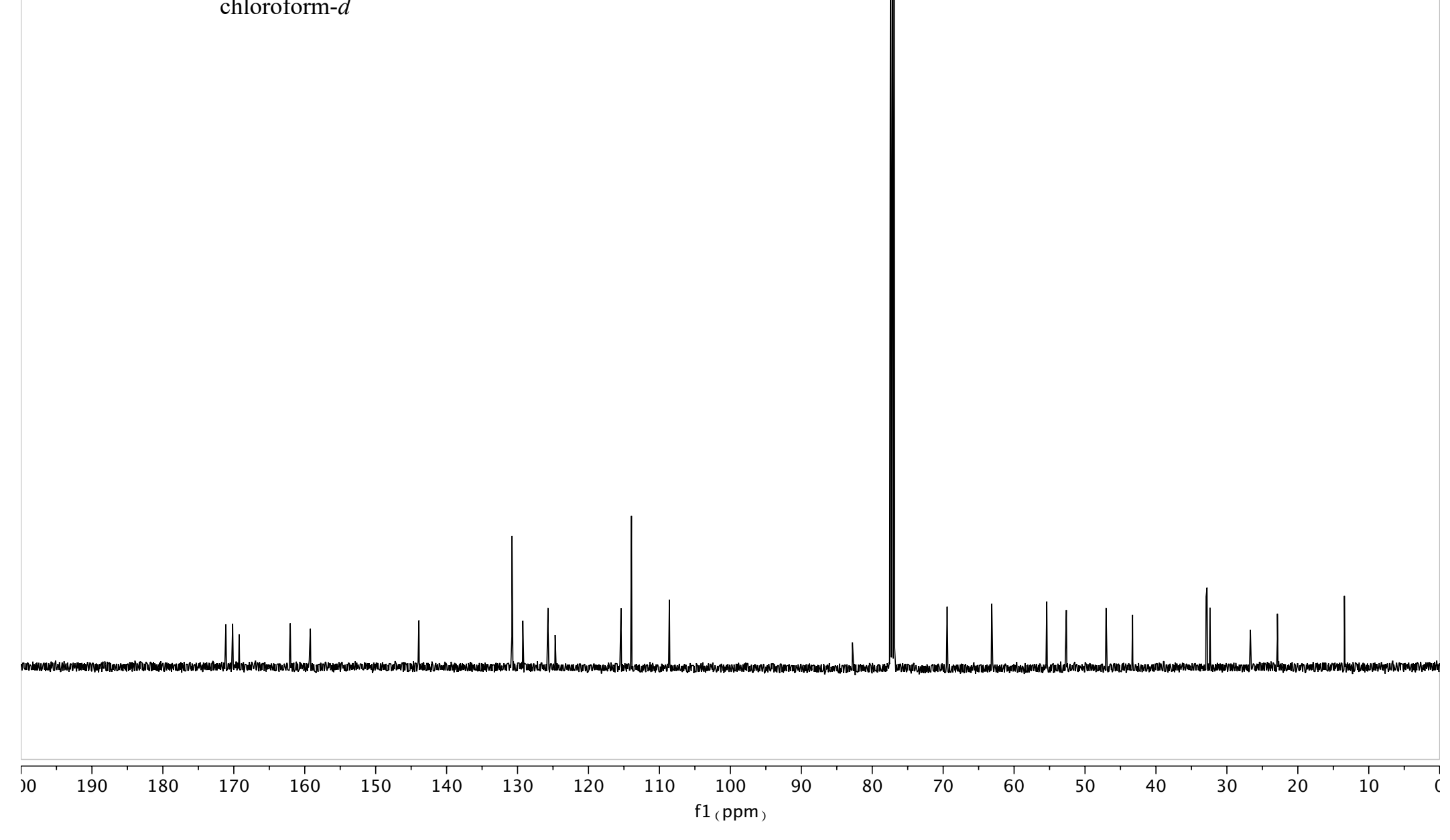




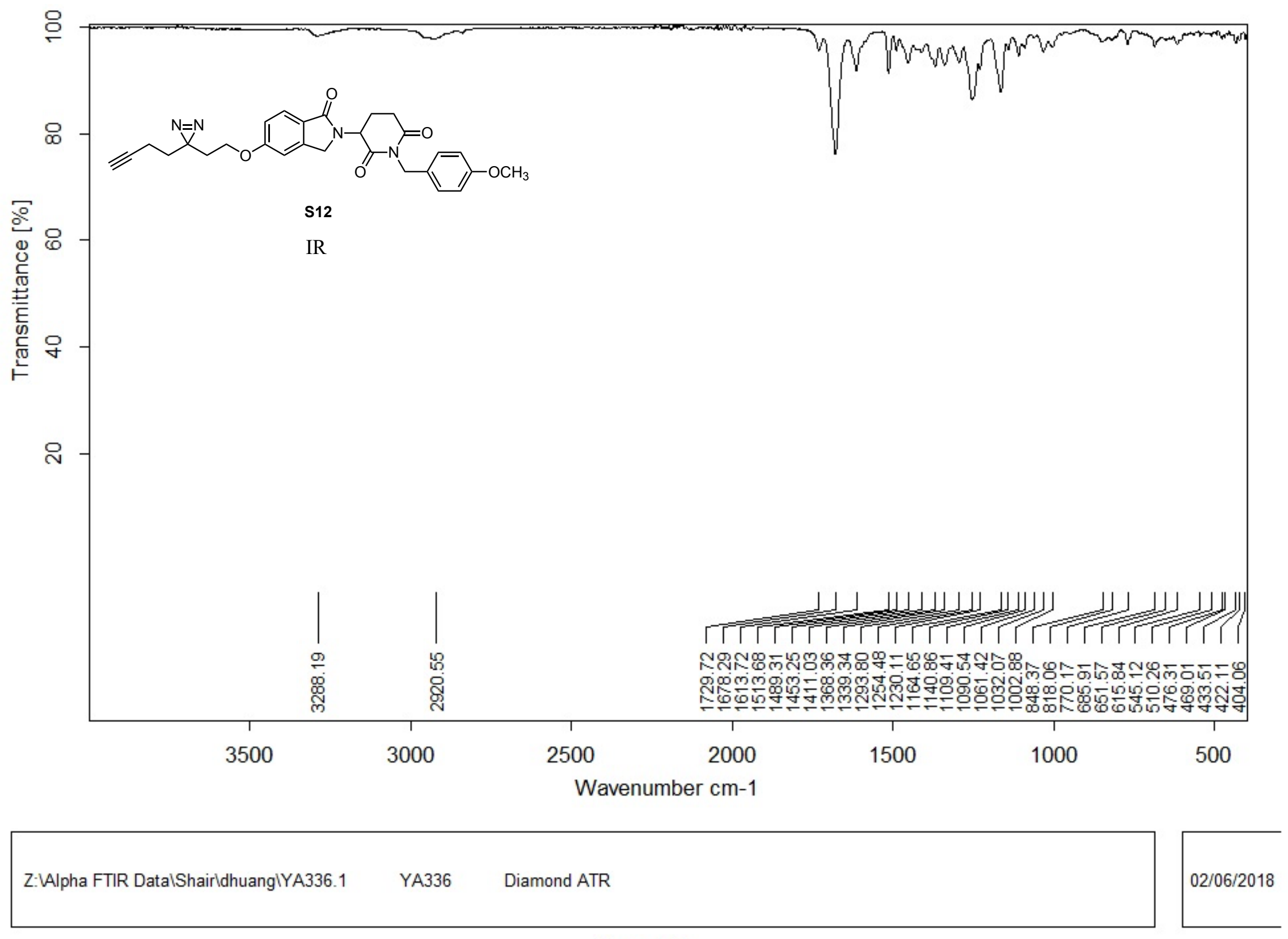

Page 1/2 


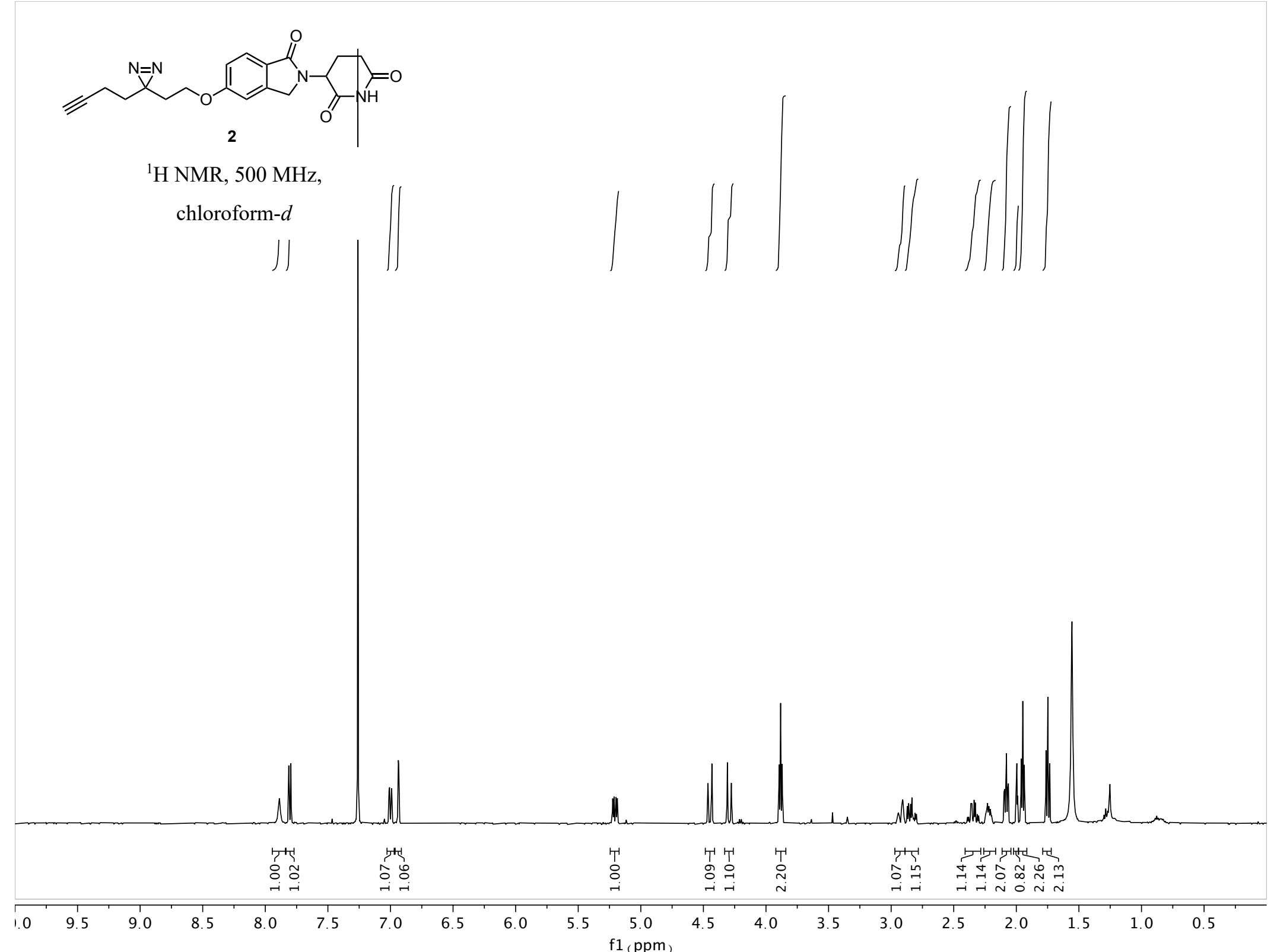




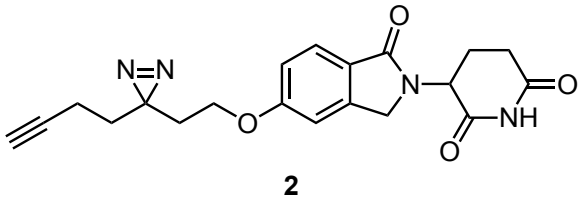

${ }^{13} \mathrm{C}$ NMR, $125 \mathrm{MHz}$,

chloroform- $d$
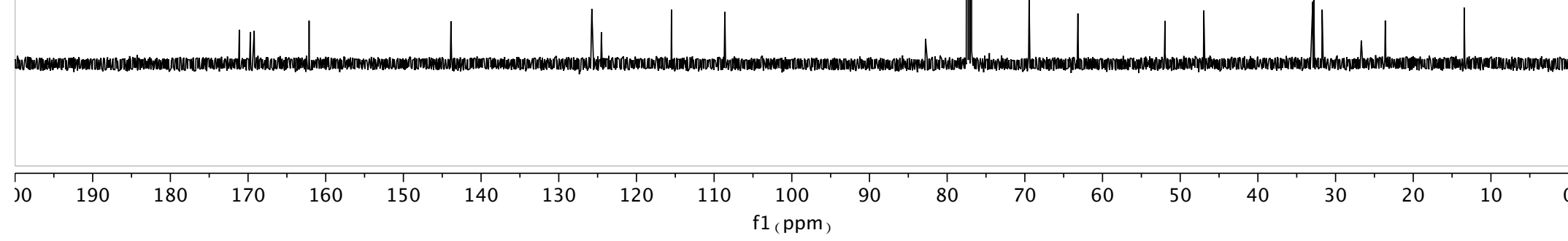


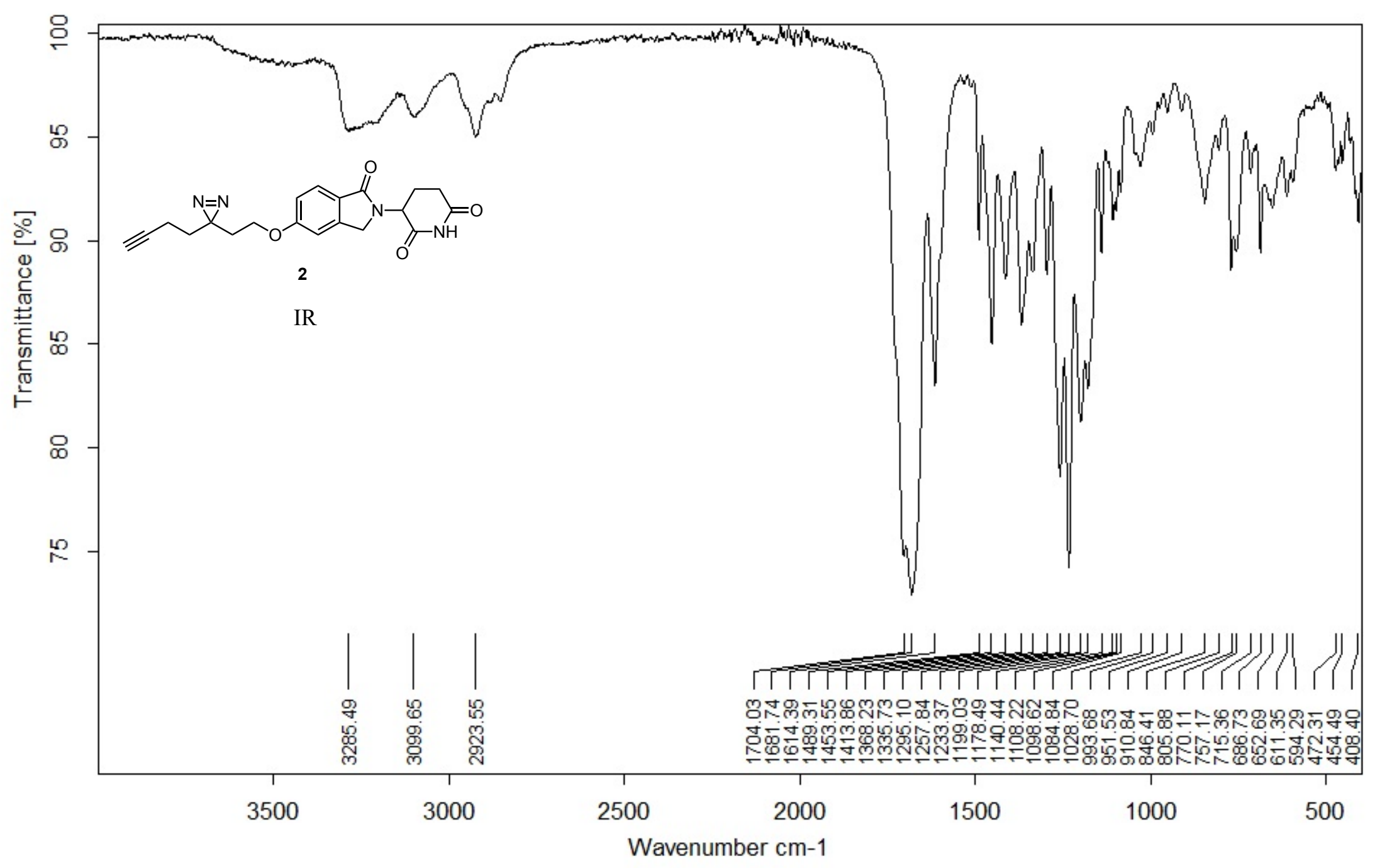

Z: :Alpha FTIR DatalWoolYukalYA340.0

YA340

Diamond ATR 


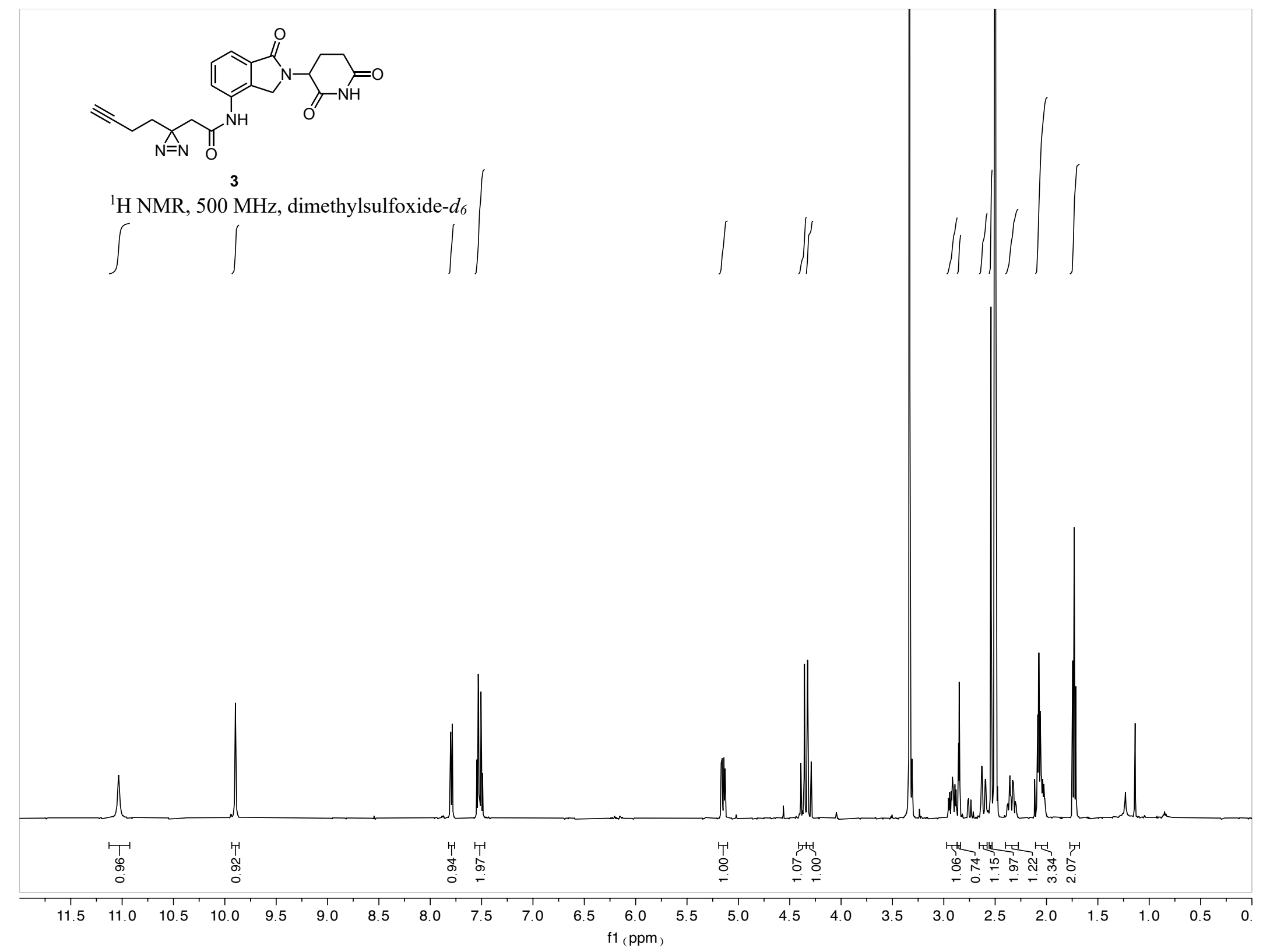




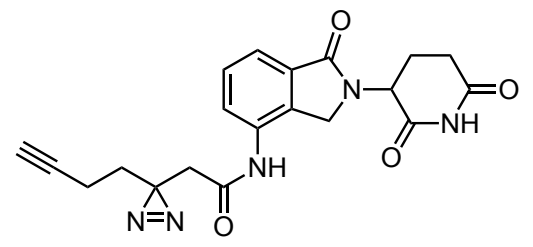

3

${ }^{13} \mathrm{C}$ NMR, $125 \mathrm{MHz}$, dimethylsulfoxide- $d_{6}$

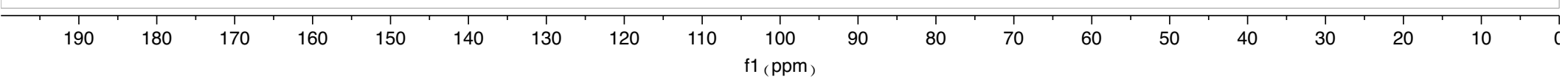




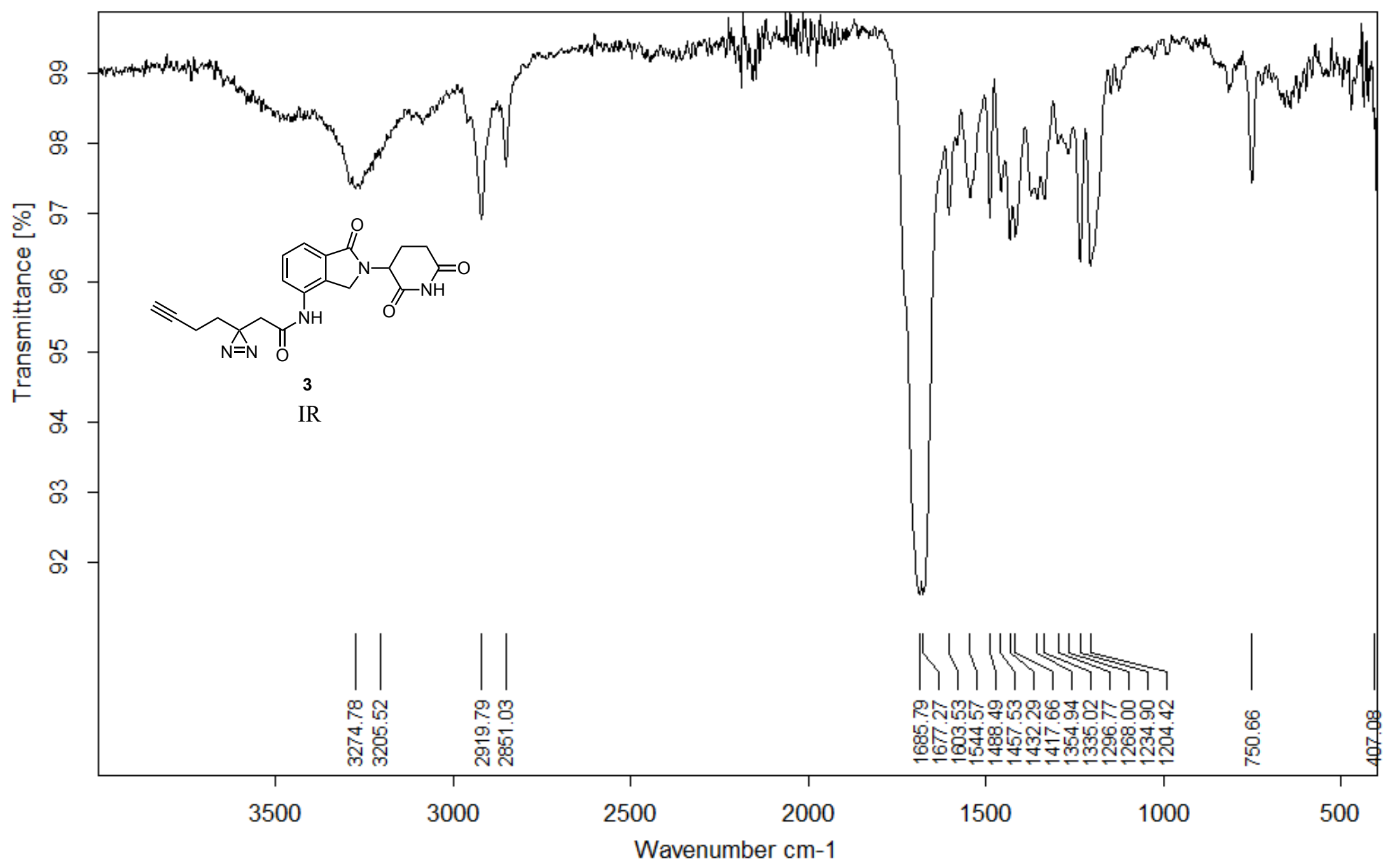

Z:Alpha FTIR DatalWoolYukalYA241 NpLEN.1 YA241 NpLEN

Diamond ATR

09/06/2018 


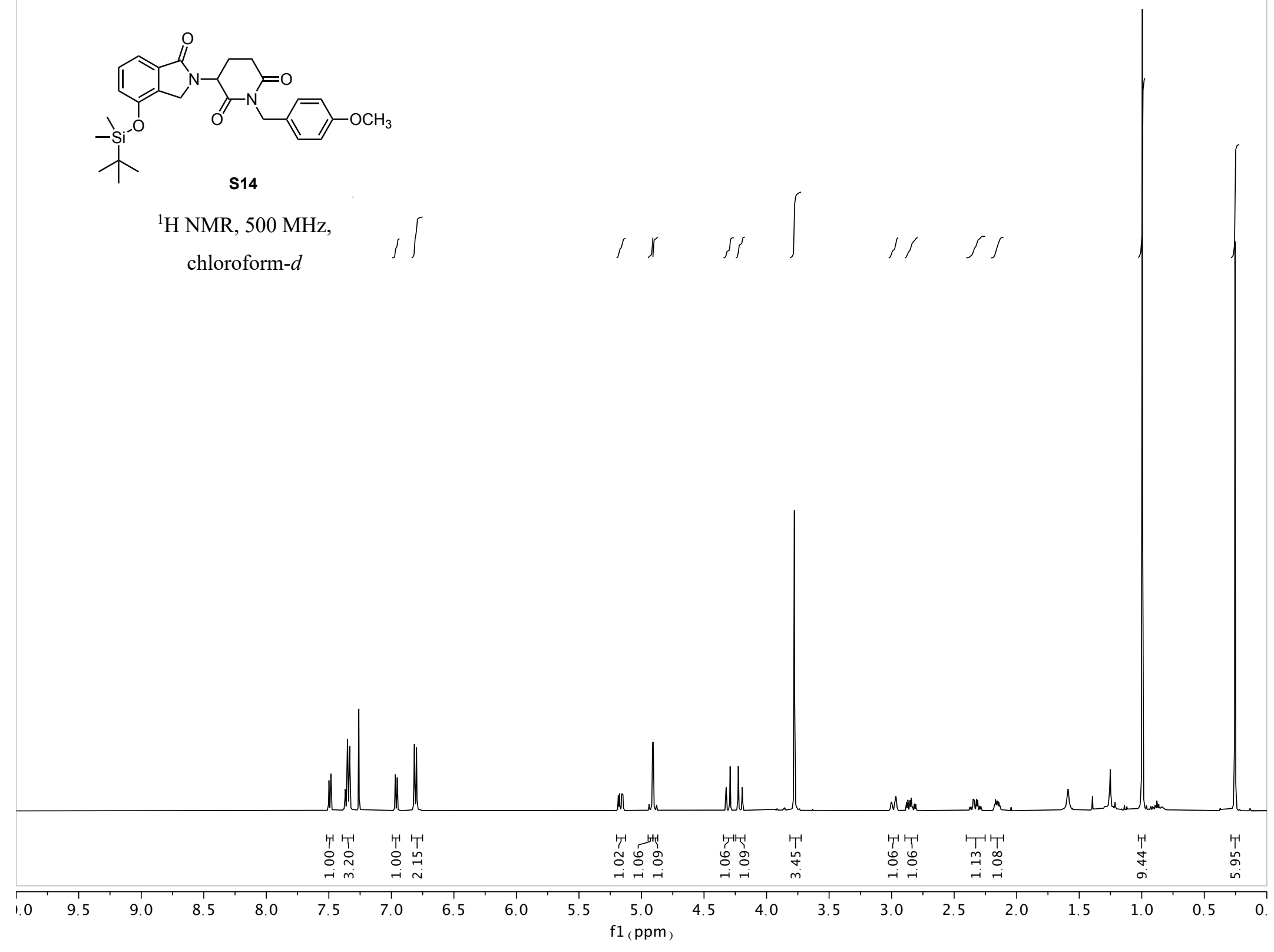




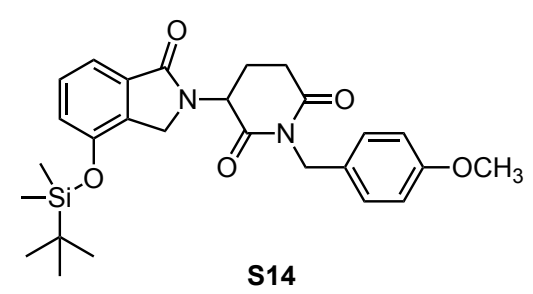

${ }^{13} \mathrm{C}$ NMR, $125 \mathrm{MHz}$,

chloroform- $d$ 


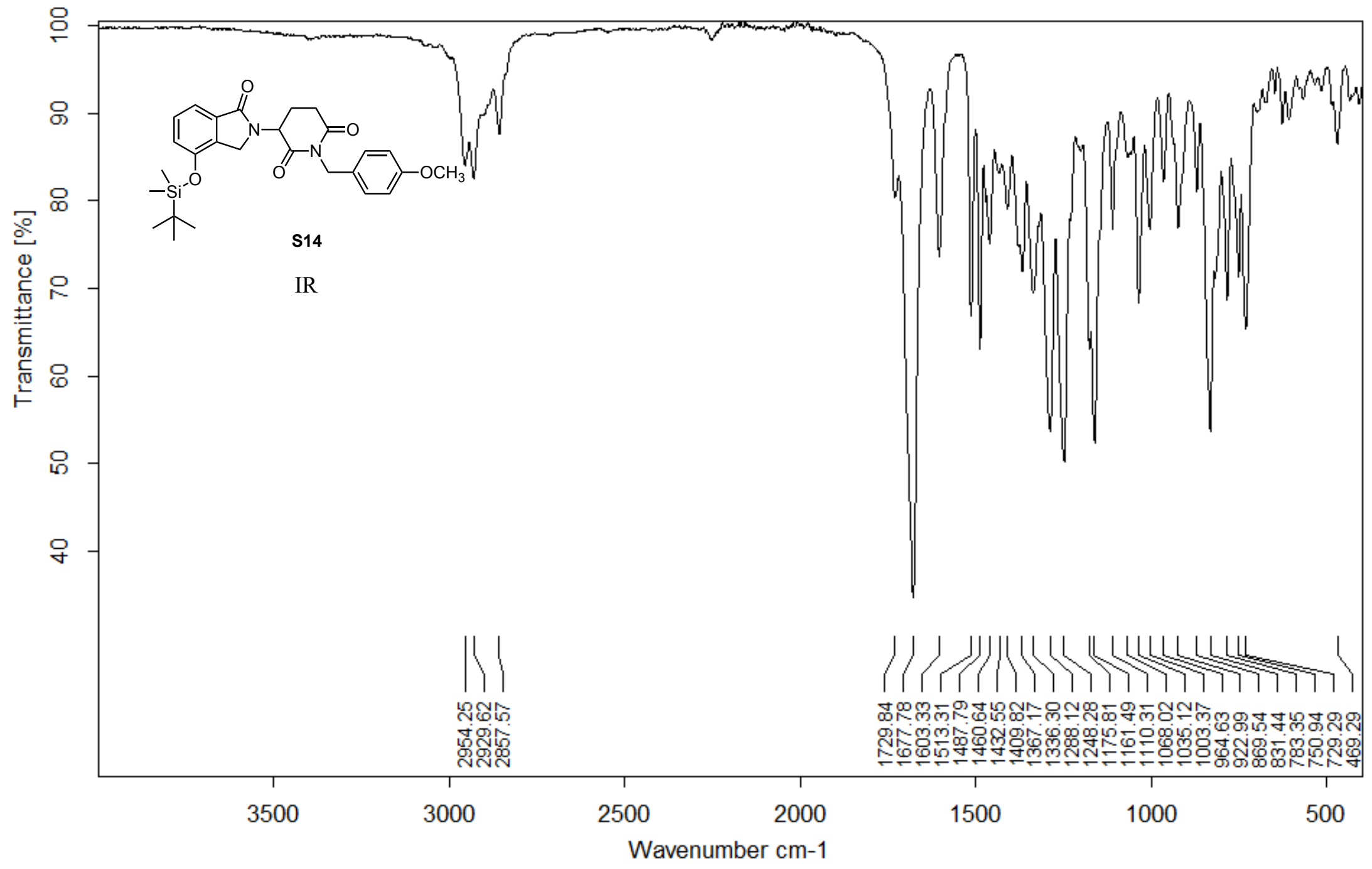

Z:MLPHA FTIR DATAIWoolYukalYA511.0 YA511 Diamond ATR 


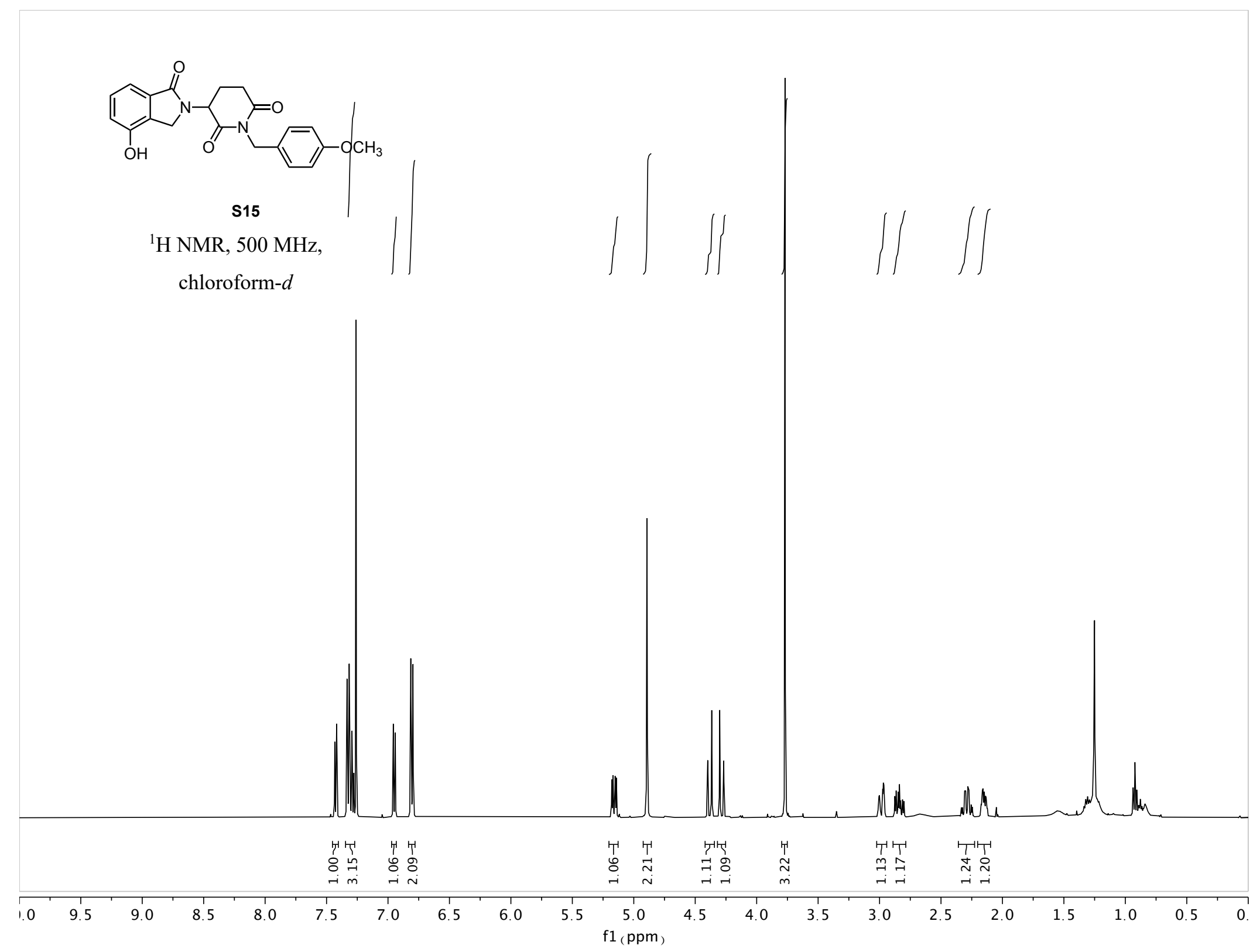



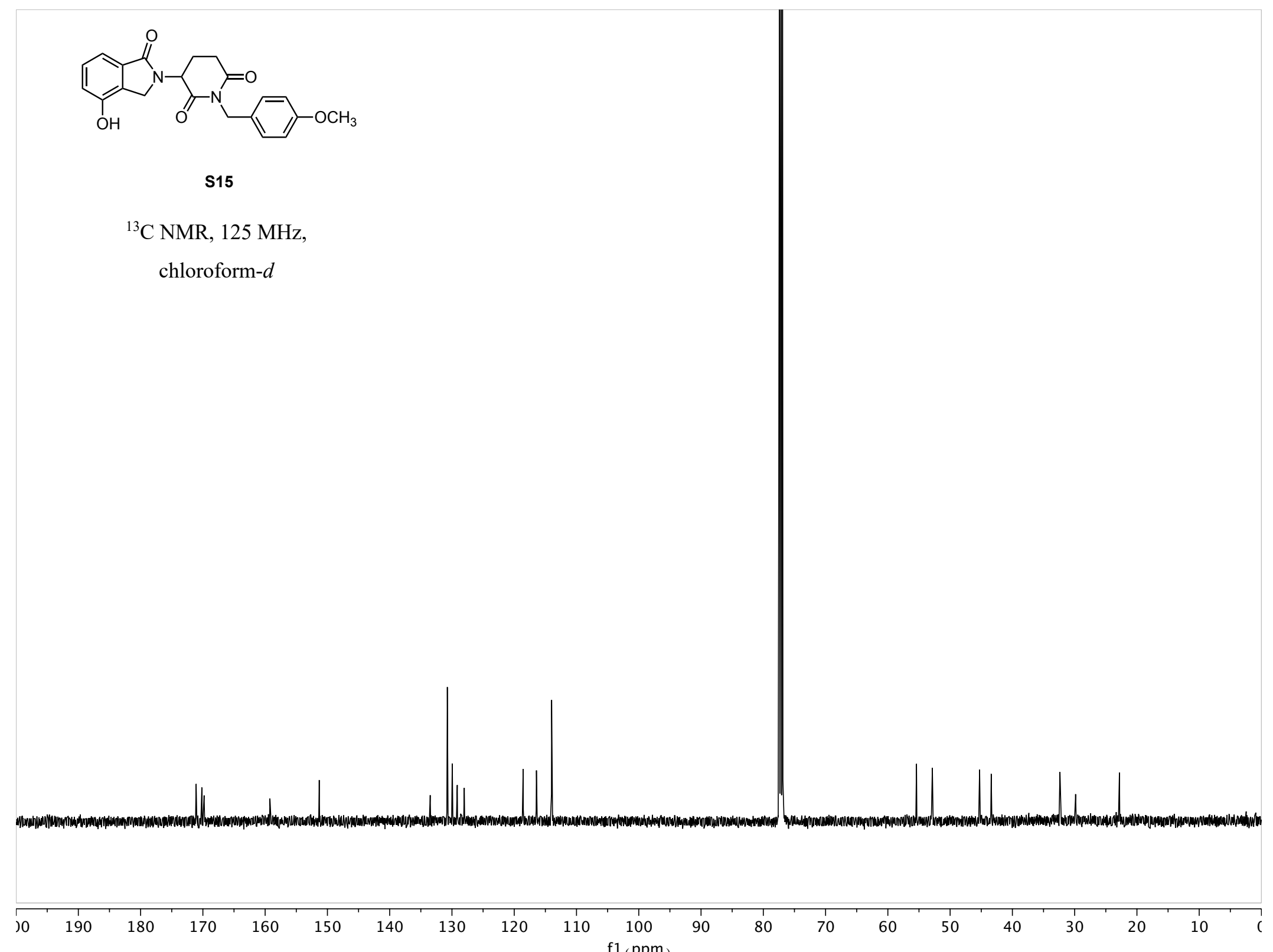


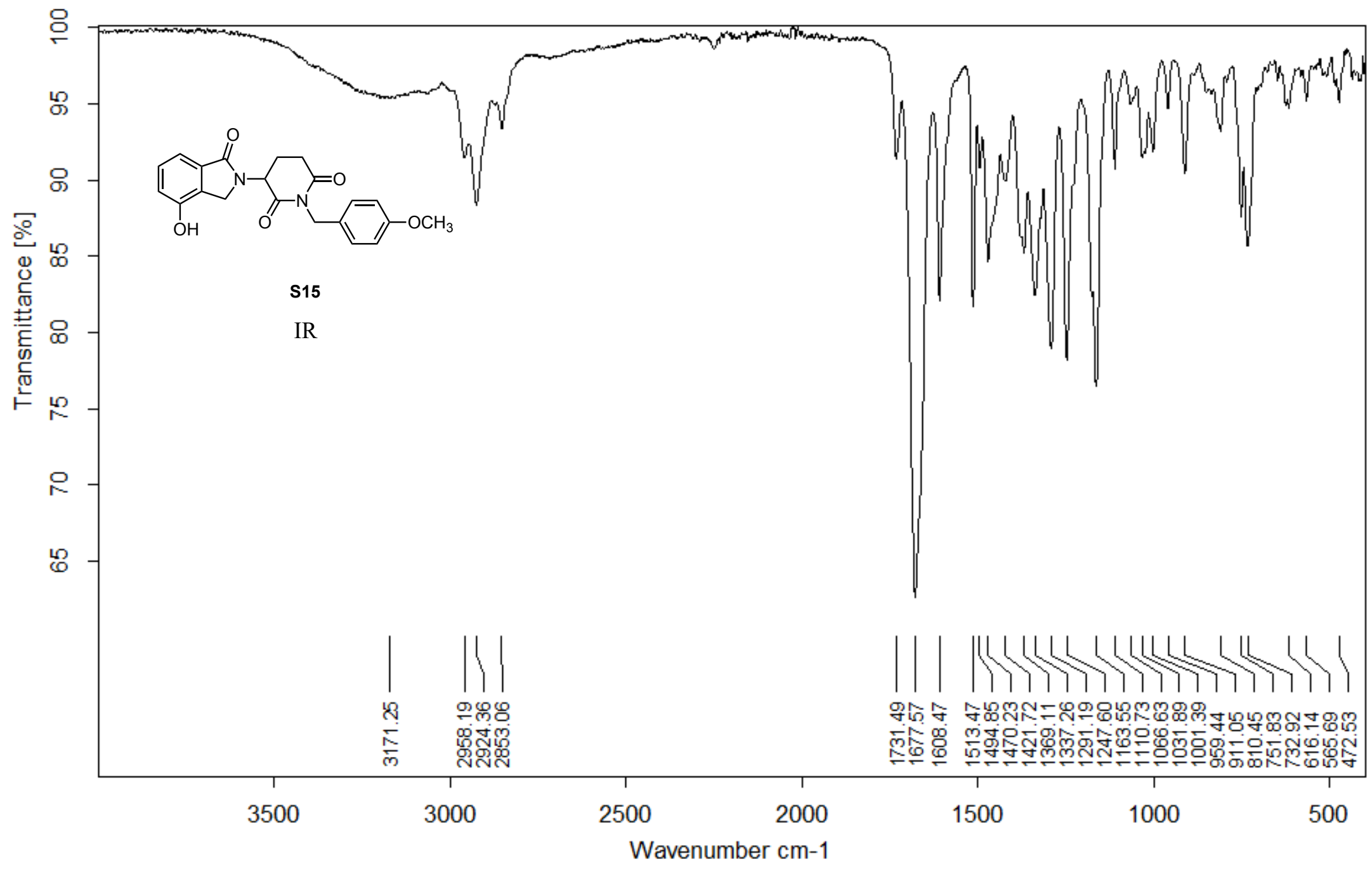

Z:VALPHA FTIR DATAIWoolYukalYA520.0 $\quad$ YA520 Diamond ATR




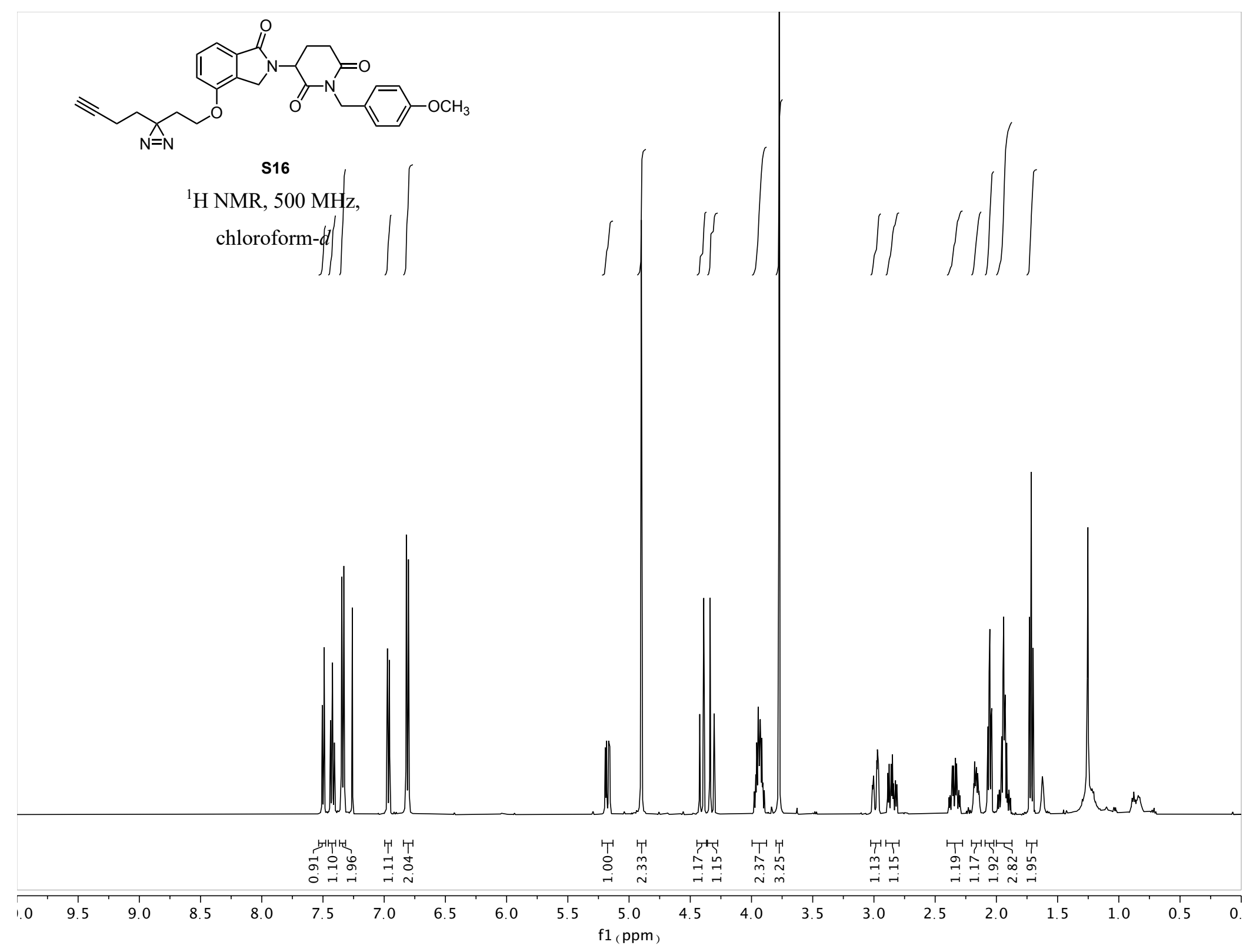




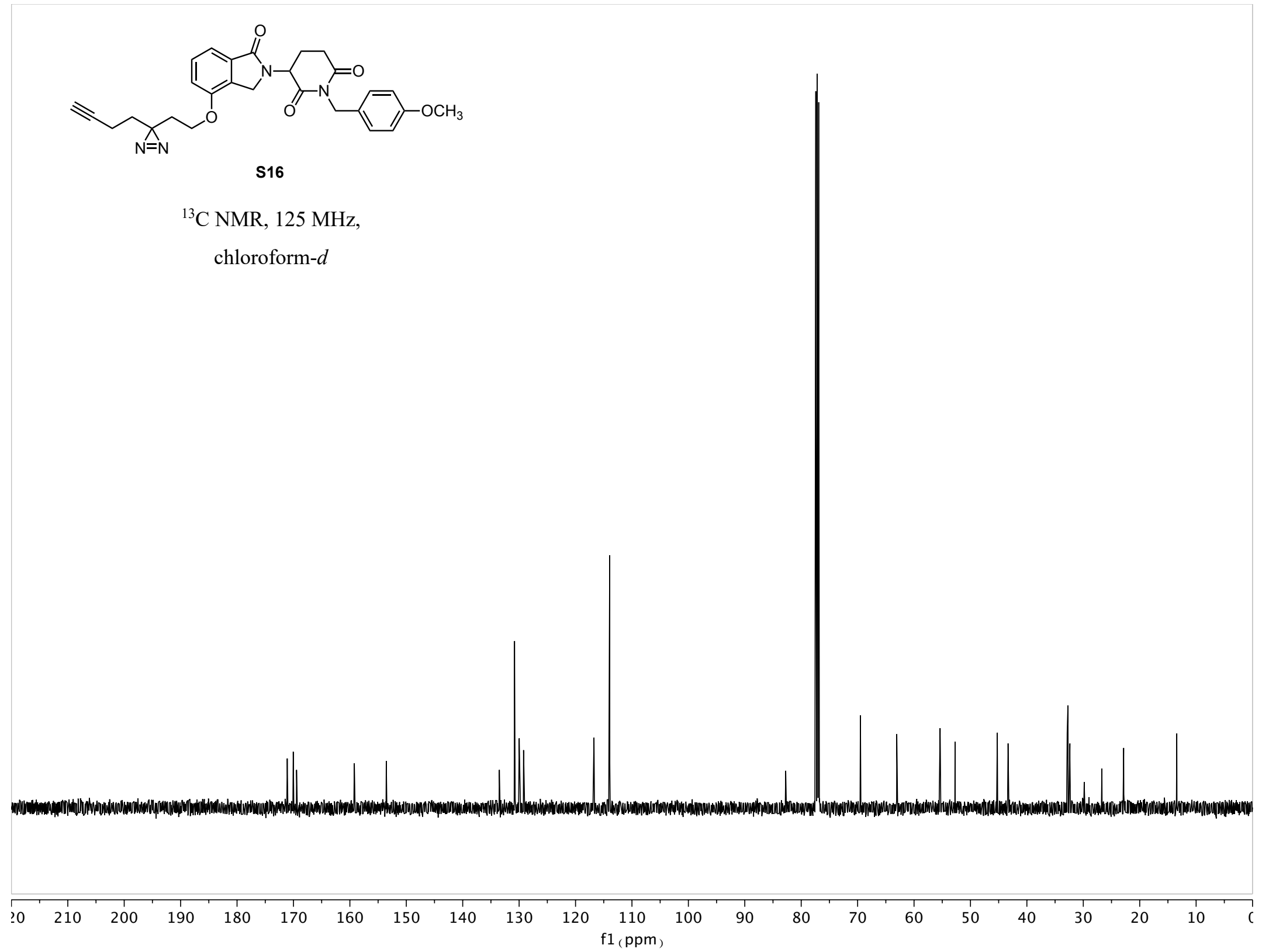

S80 


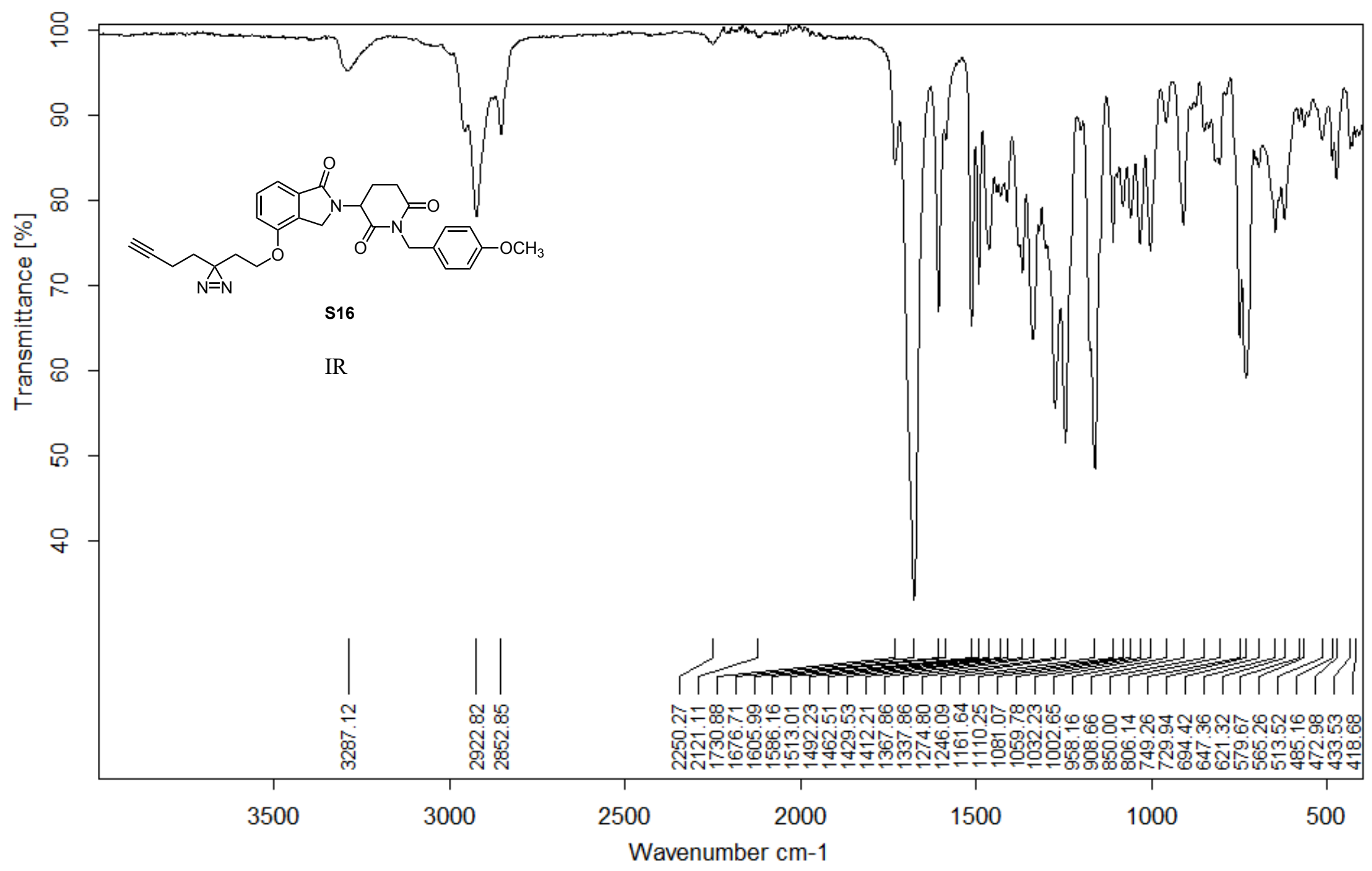

Z: Alpha FTIR DatalWoolYukalYA225-tm.0 YA225-tm Diamond ATR 


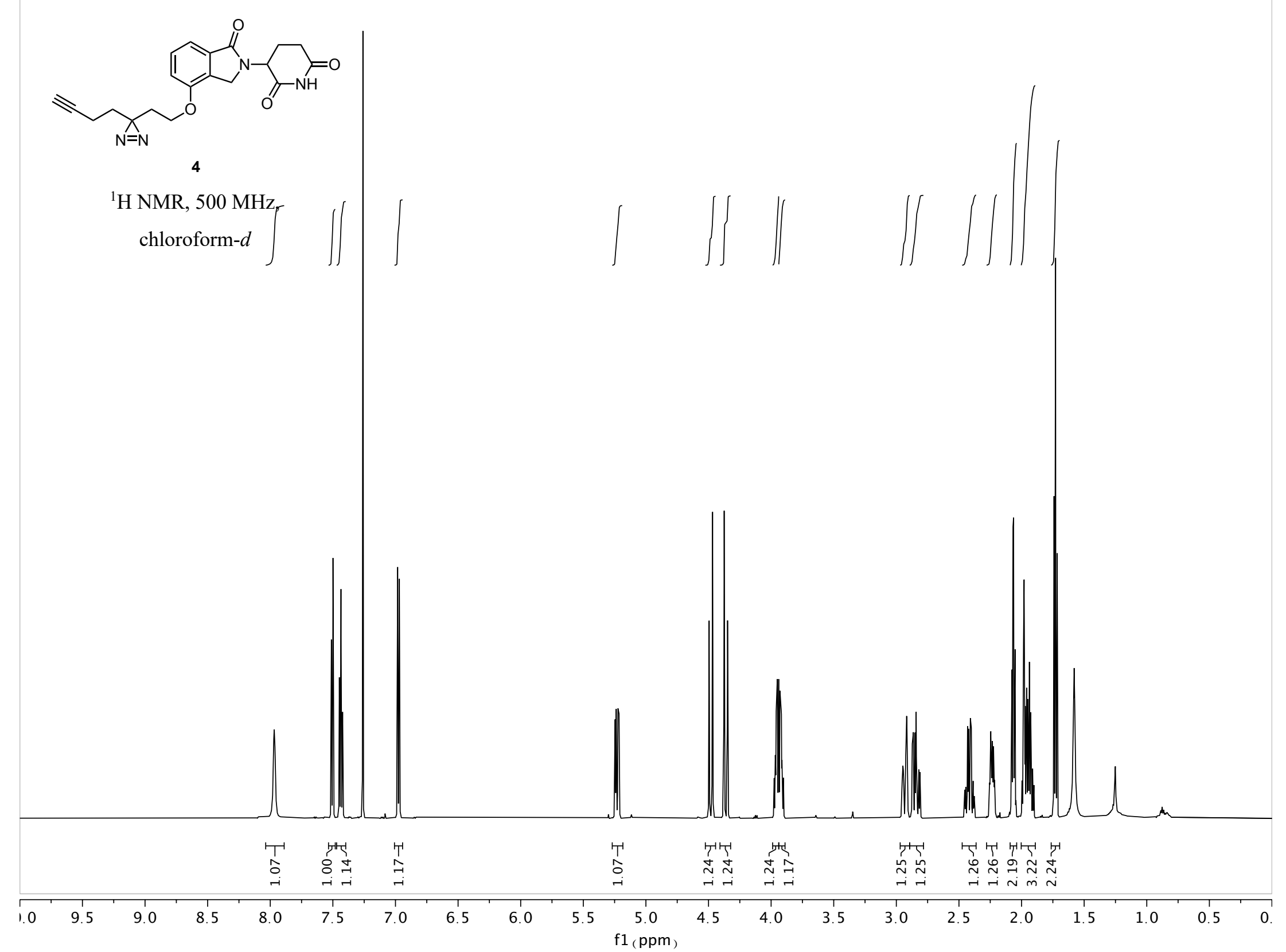




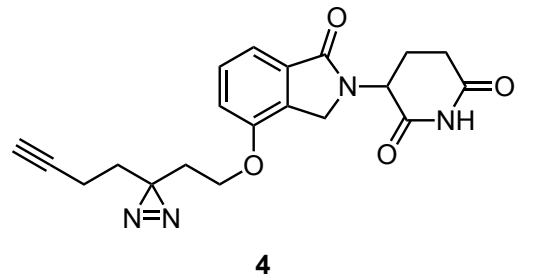

${ }^{13} \mathrm{C} \mathrm{NMR}, 125 \mathrm{MHz}$,

chloroform-d
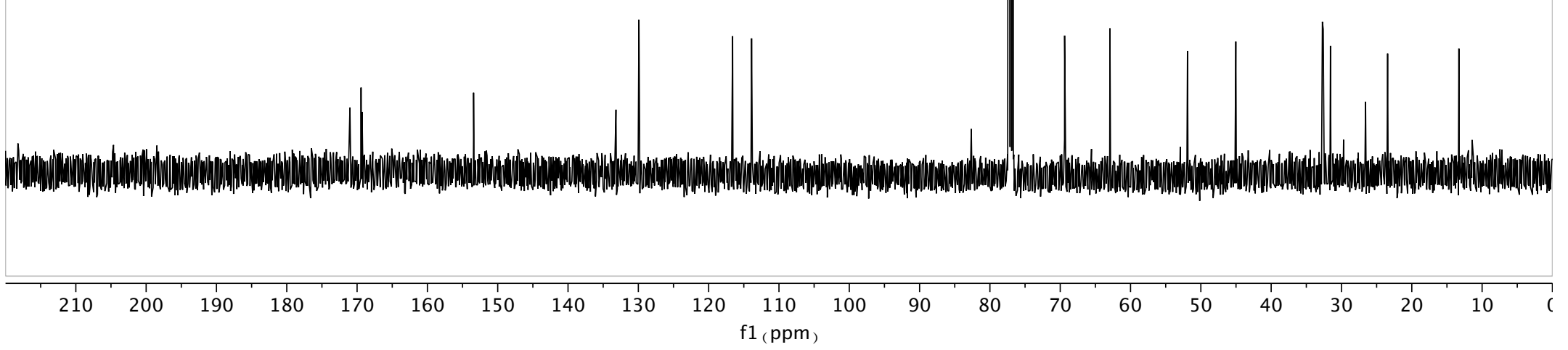


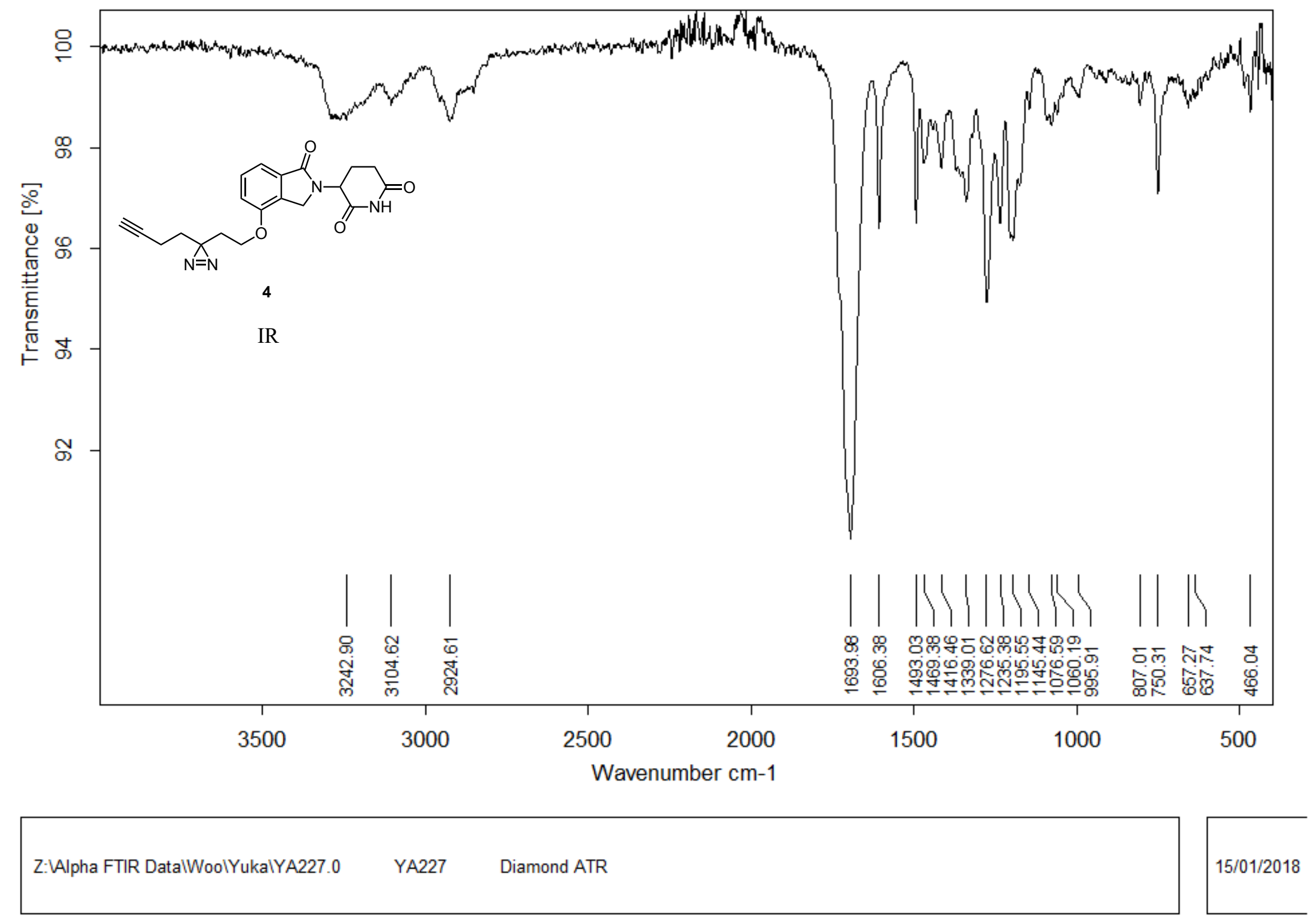

Page 1/2 


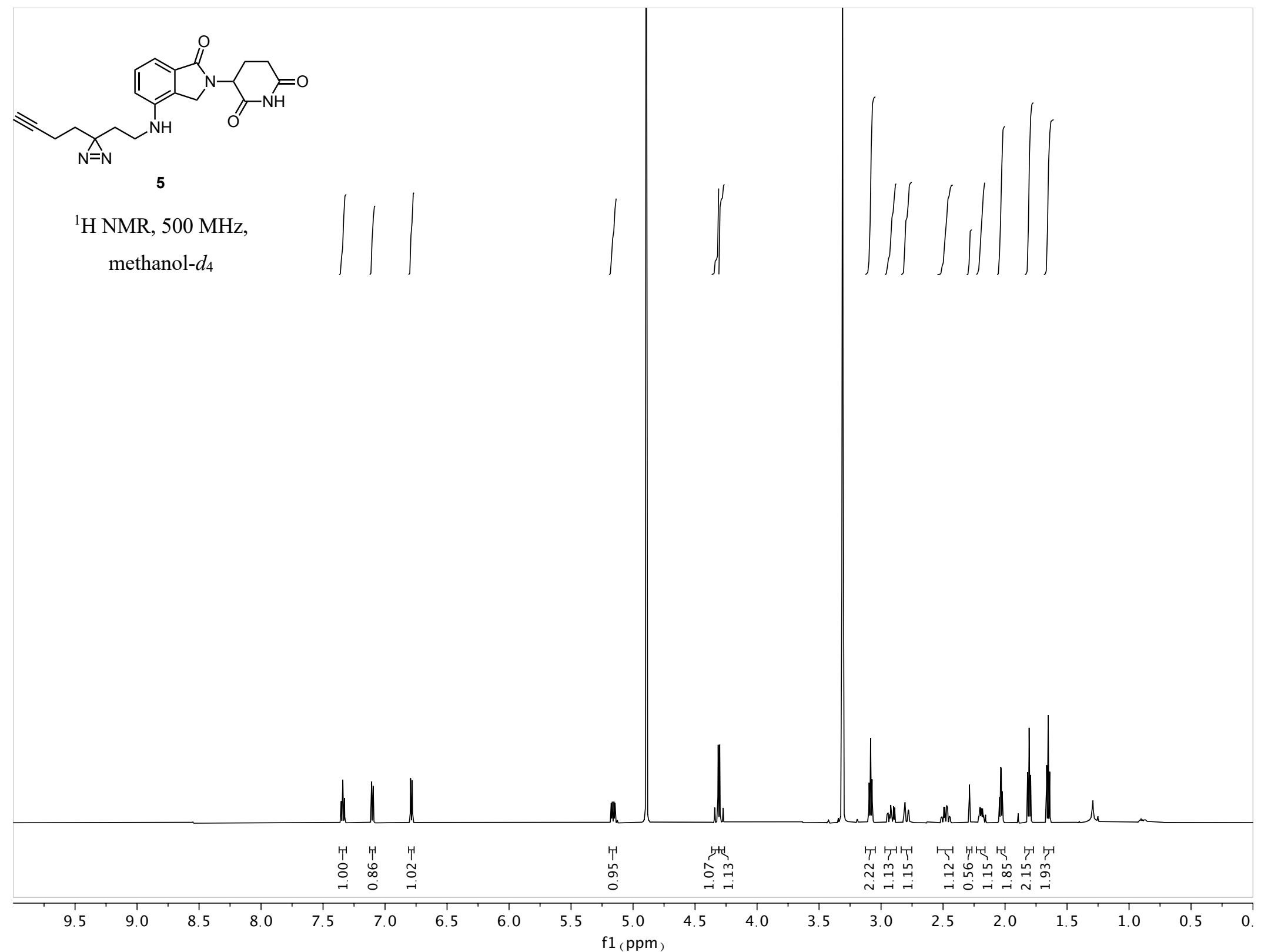




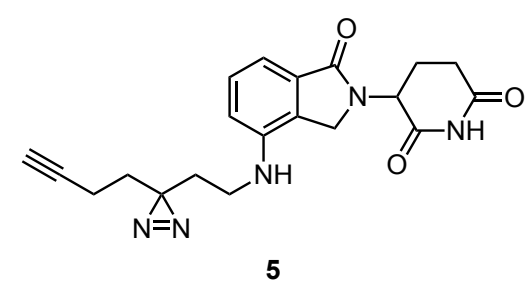

${ }^{13} \mathrm{C}$ NMR, $125 \mathrm{MHz}$,

methanol- $d_{4}$ 


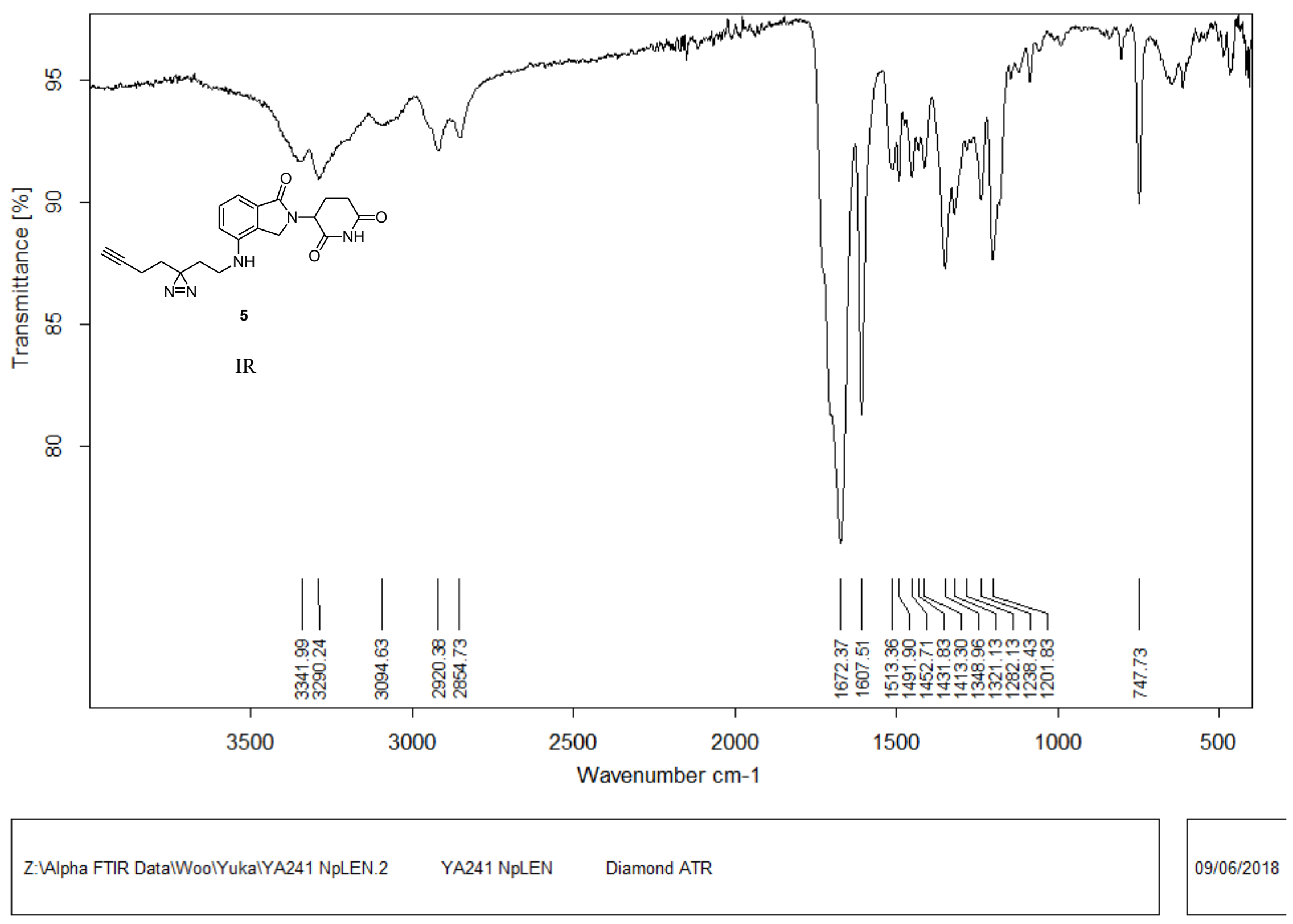

Page 1/2 


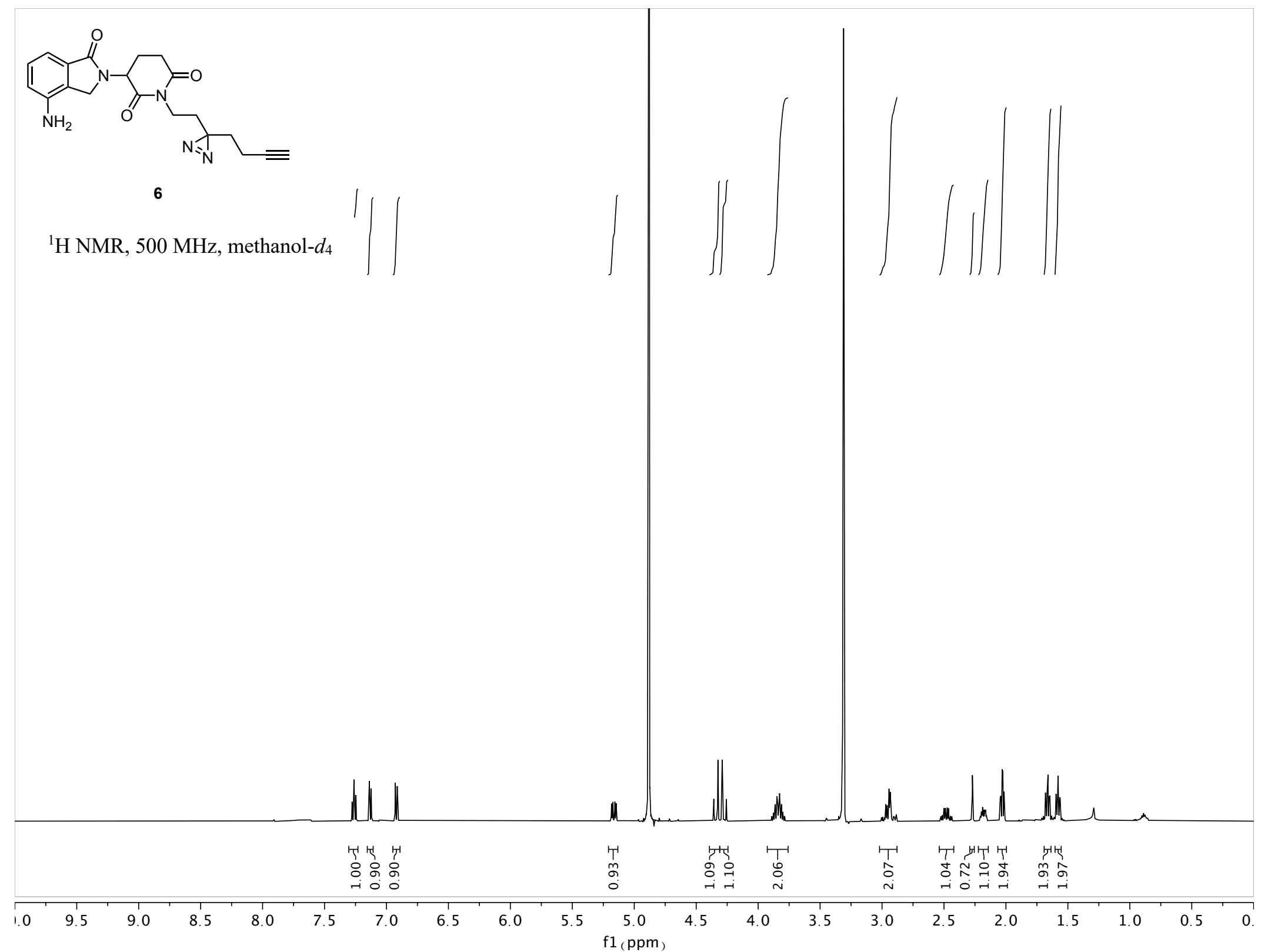




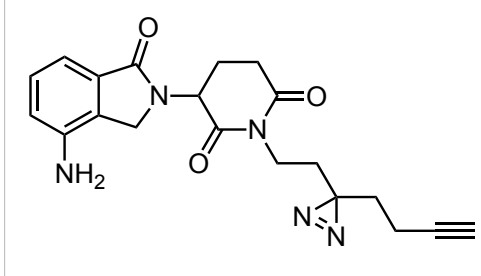

6

${ }^{13} \mathrm{C}$ NMR, $125 \mathrm{MHz}$,

chloroform- $d$
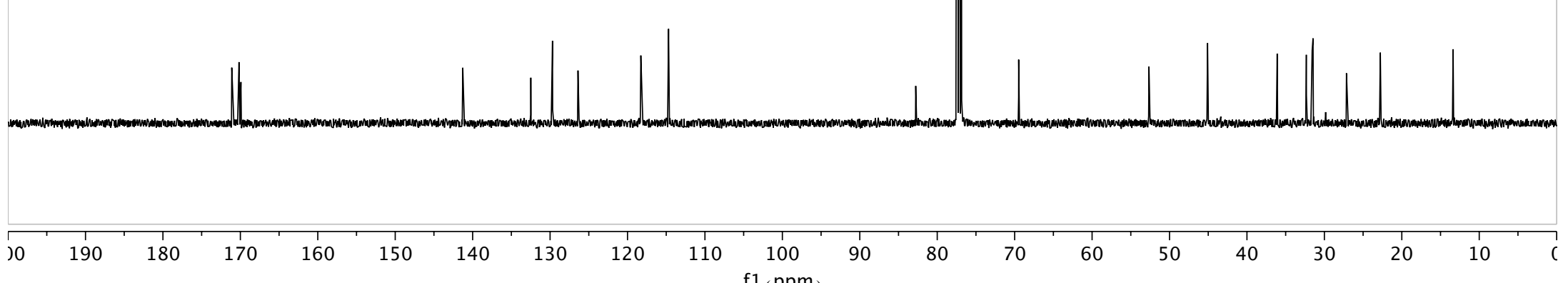


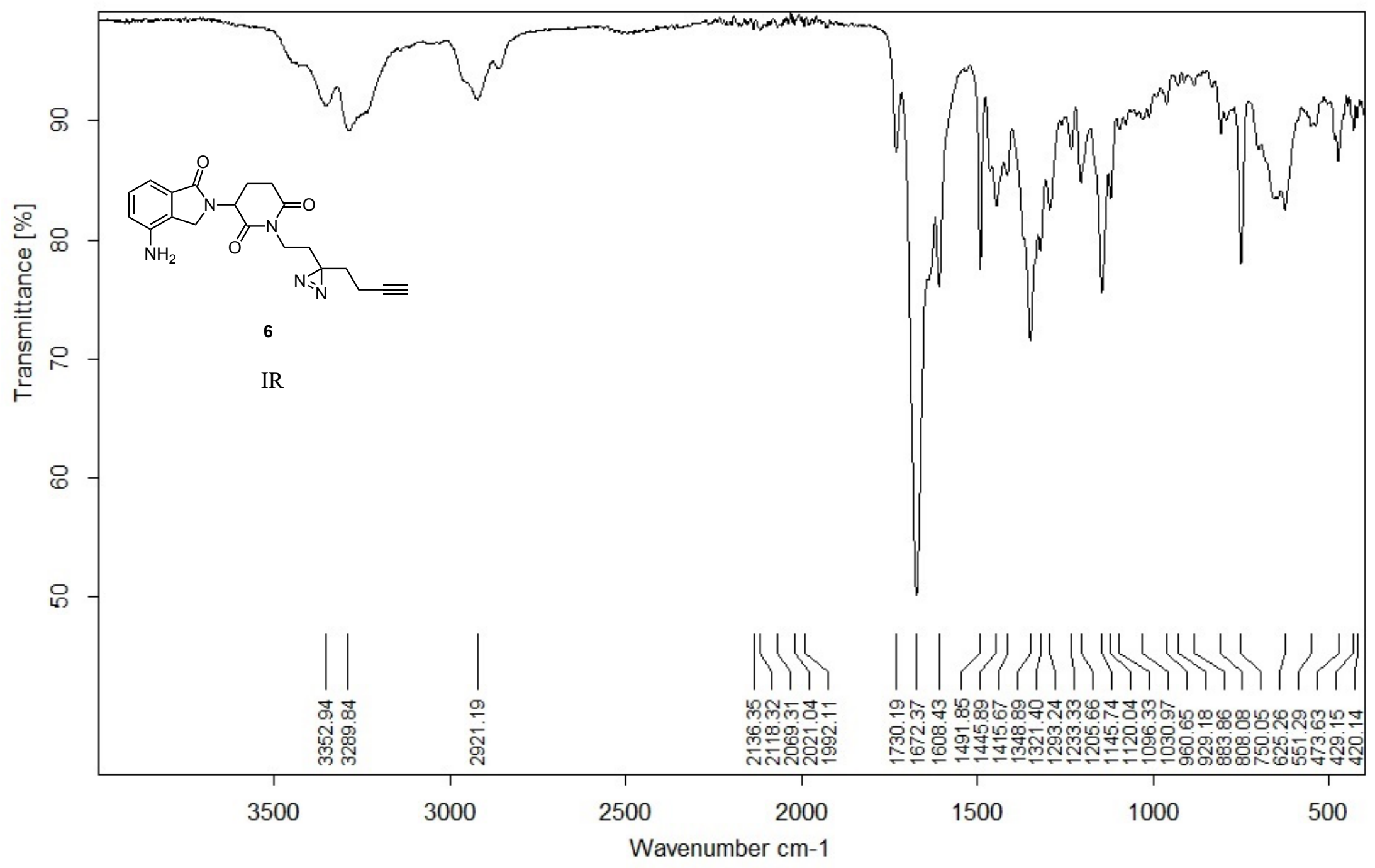

Z:IALPHA FTIR DATAIWoolYukalYA280.0

YA280

Diamond ATR 


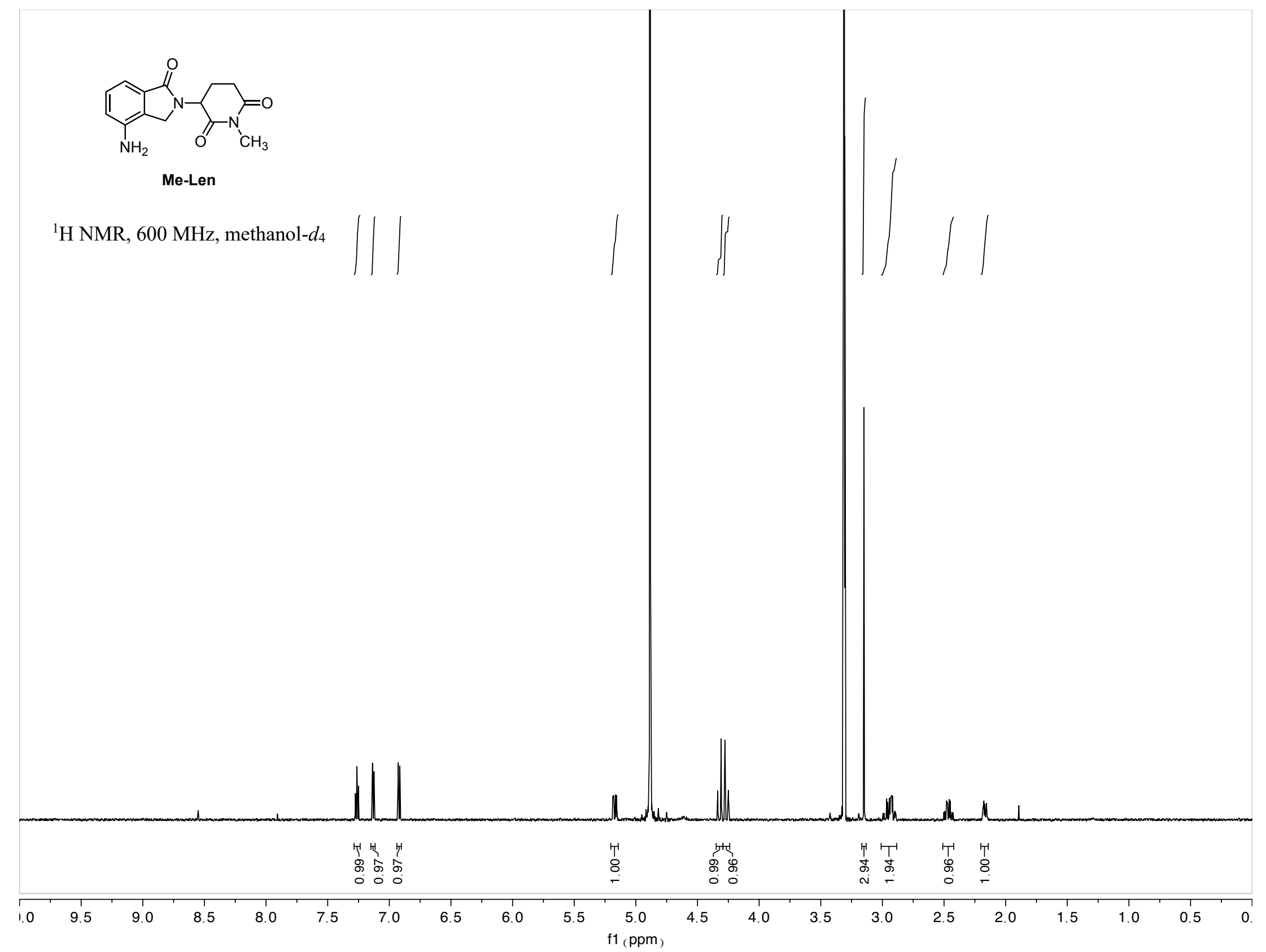



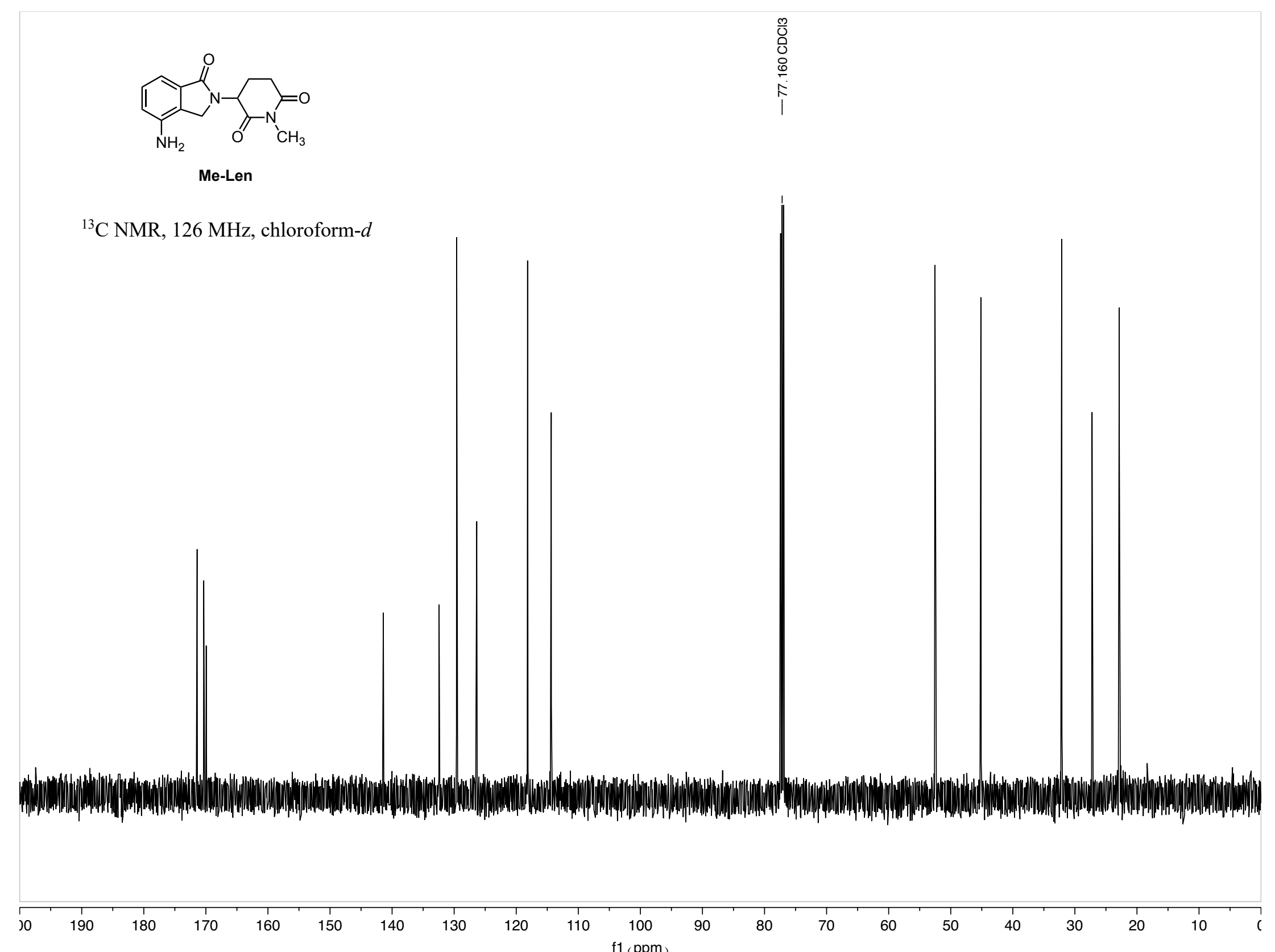


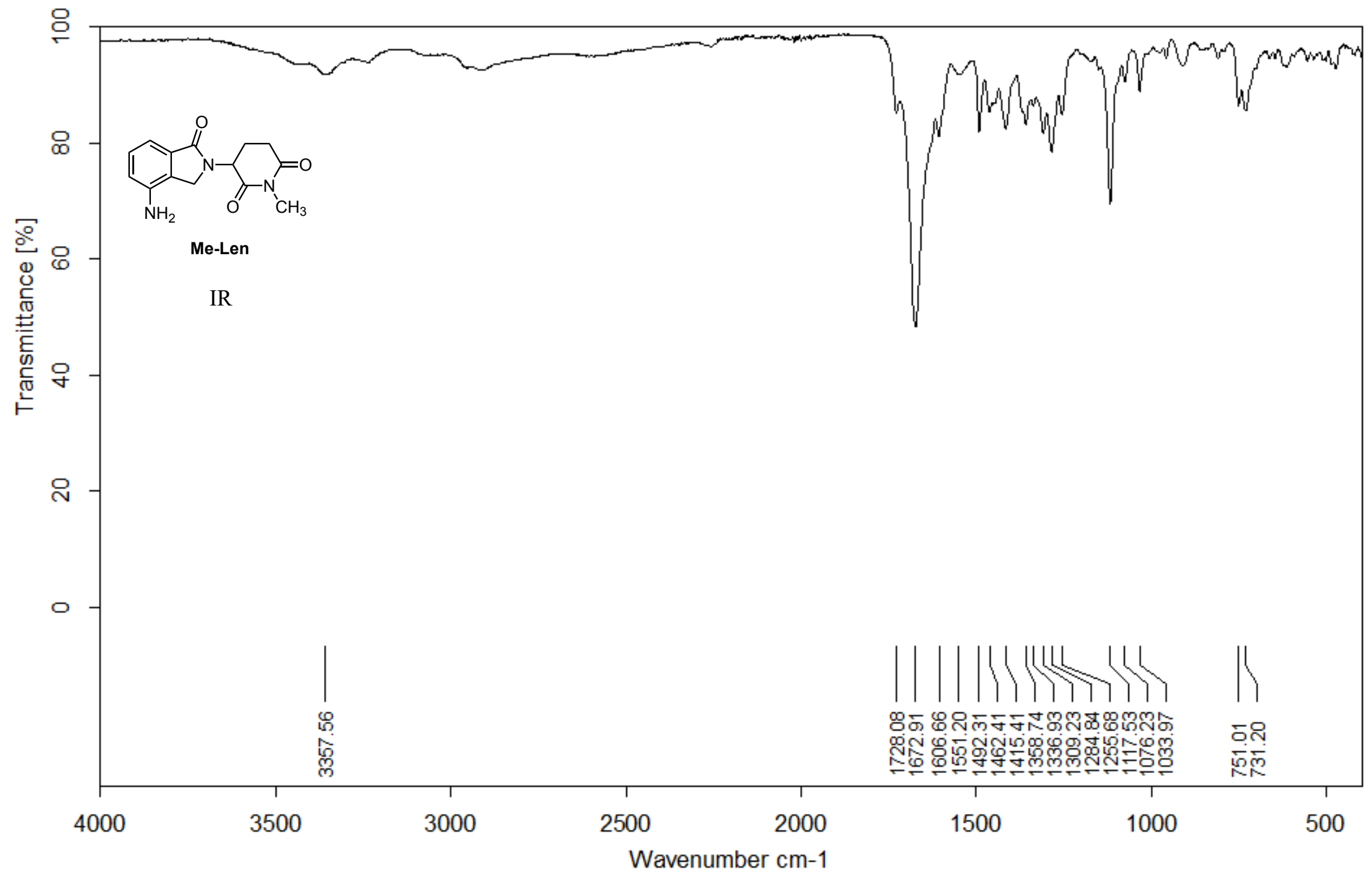

Z:IWoolYukalYA459.0 YA459 Diamond ATR 


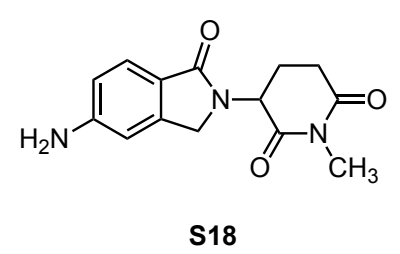

${ }^{1} \mathrm{H}$ NMR, $400 \mathrm{MHz}$, dimethylsulfoxide- $d_{6}$

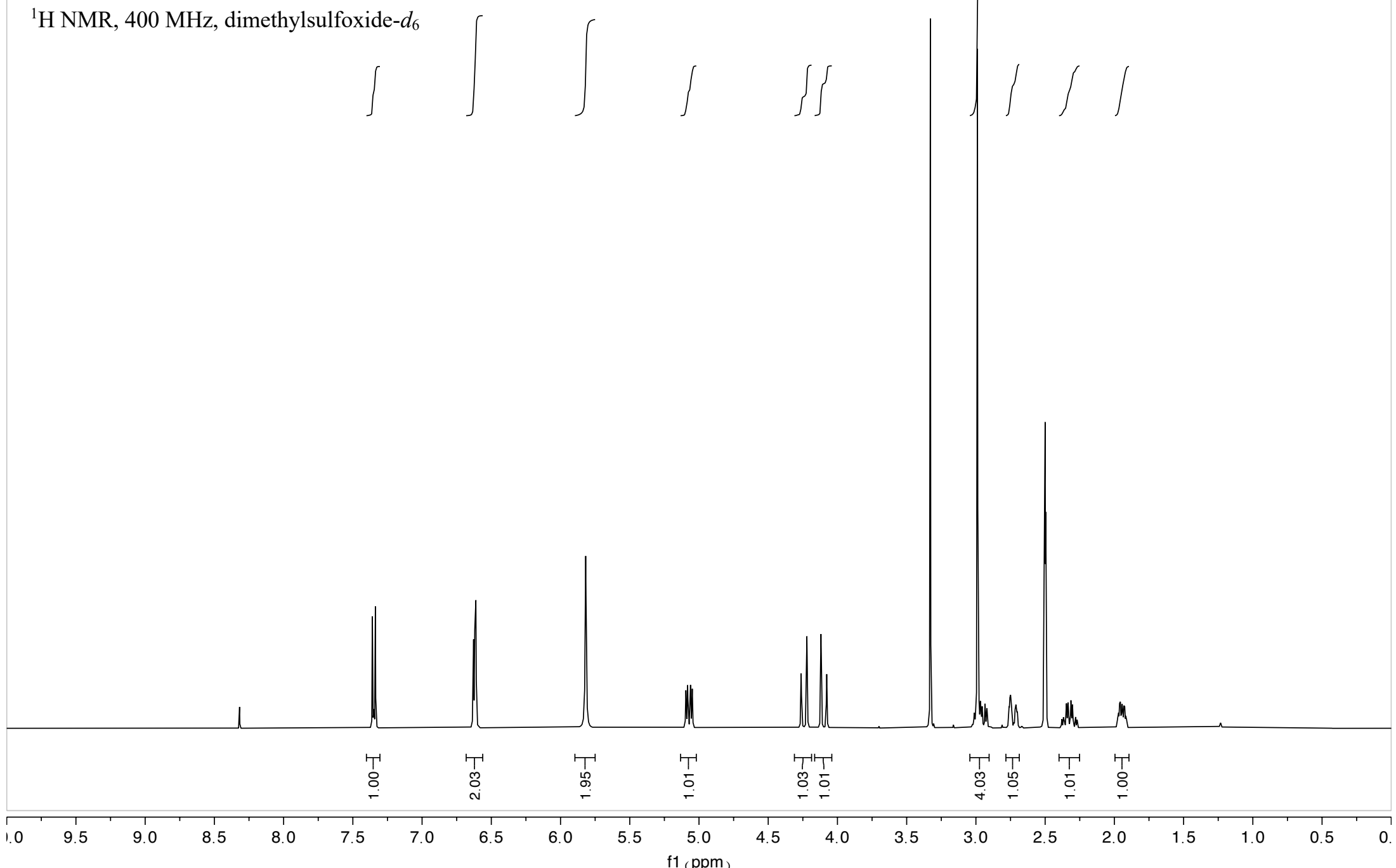




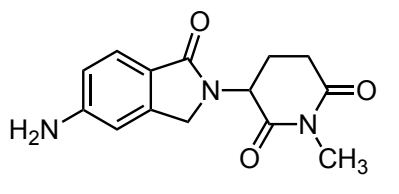

S18

${ }^{13} \mathrm{C}$ NMR, $101 \mathrm{MHz}$, dimethylsulfoxide- $d_{6}$

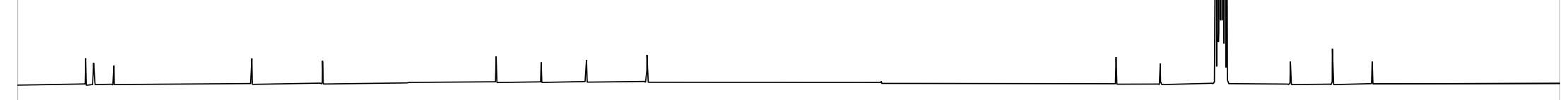

1

150

140

130

120 


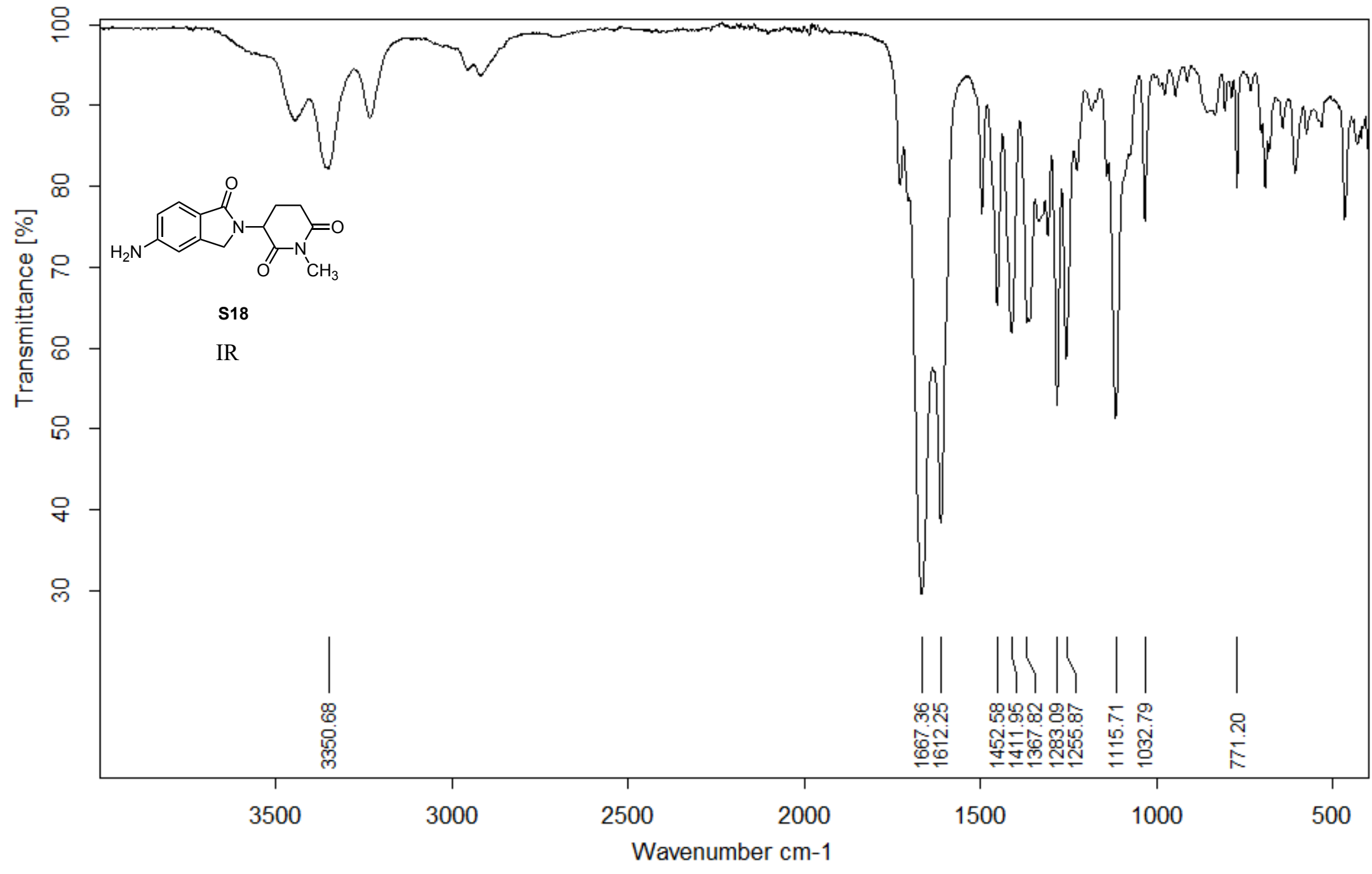

\begin{tabular}{|lll|}
\hline Z:WoolYukalYA462.0 & YA462 & Diamond ATR \\
\hline
\end{tabular}

Page 1/2 


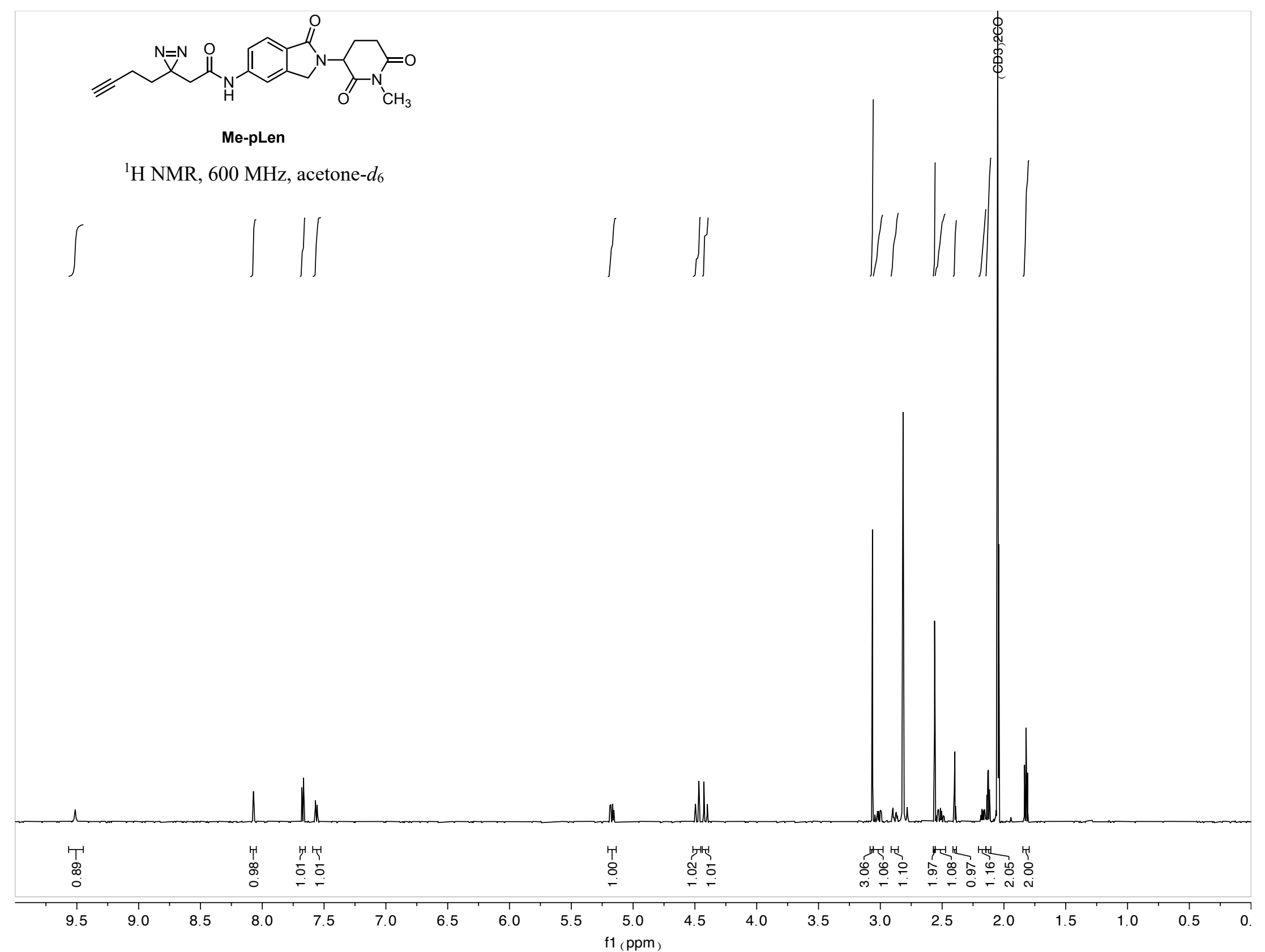



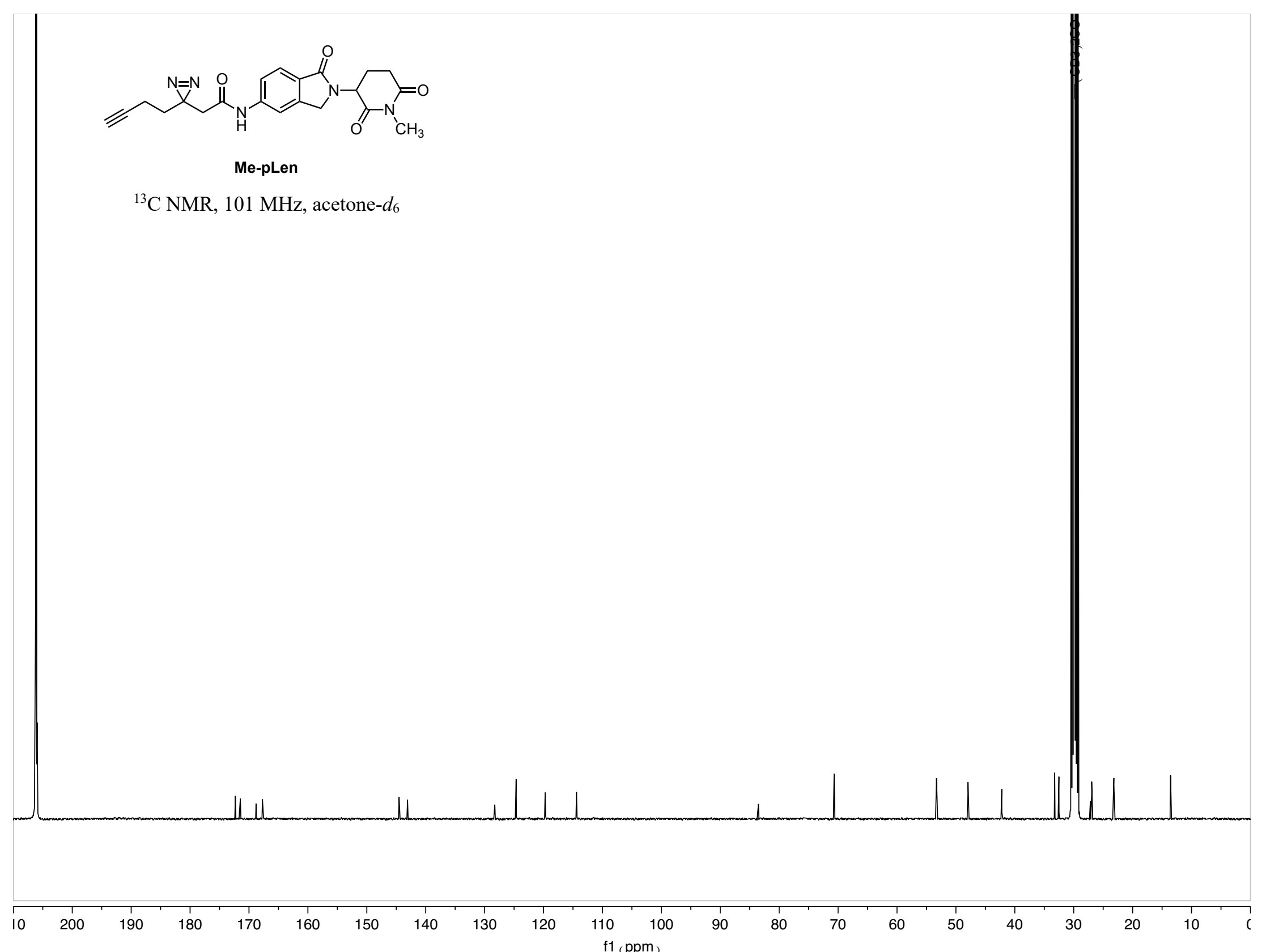


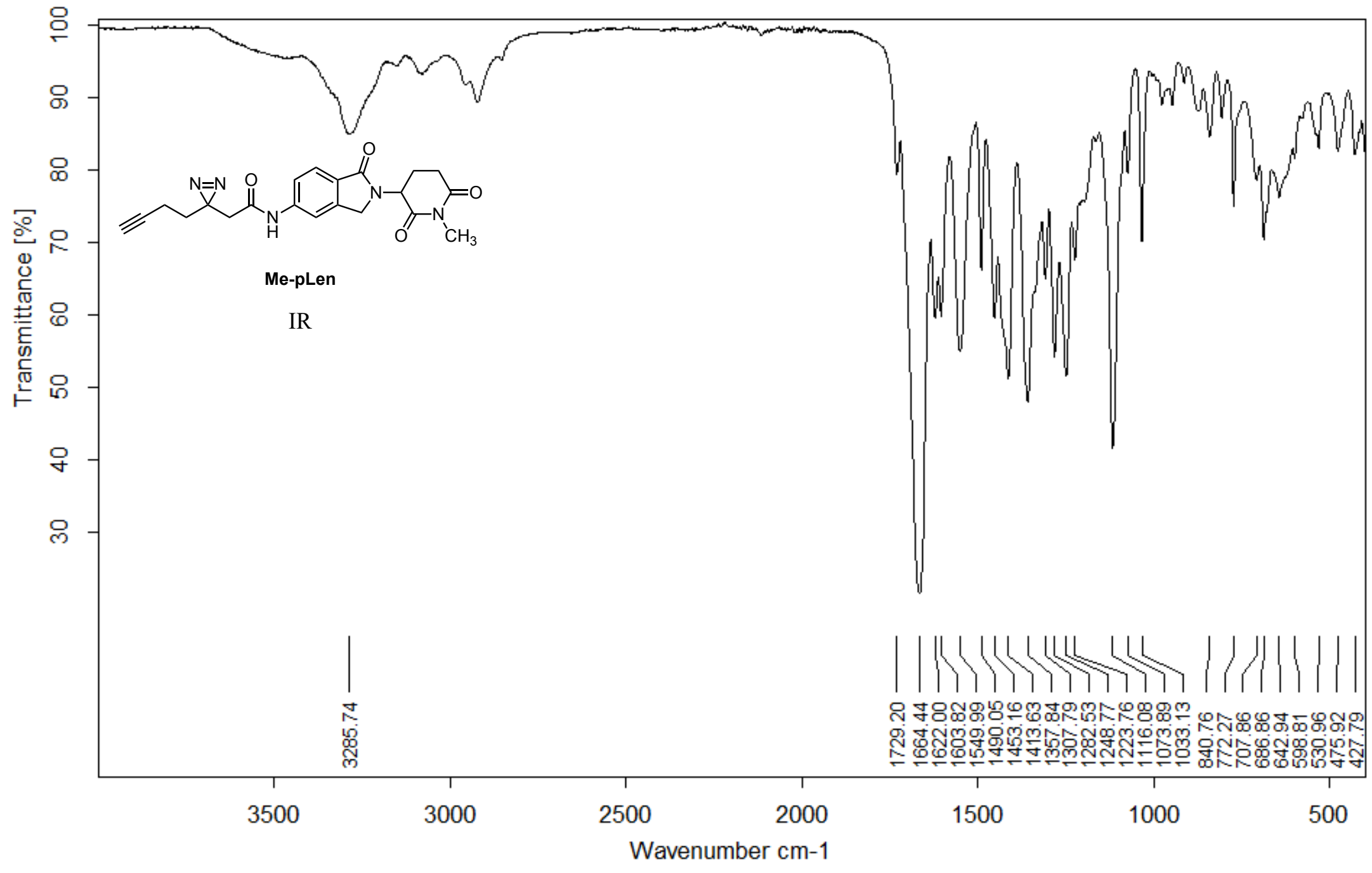

\begin{tabular}{|lll|}
\hline Z:WoolYukalYA464.0 & YA464 & Diamond ATR \\
\hline
\end{tabular}

Page 1/2 